\title{
Neurofibromatosis type 2 and central neurofibromatosis
}

\section{Leonard I. Malis, M.D.}

The Mount Sinai School of Medicine, New York City, New York

Neurofibromatosis type 2 (NF2) is a rare disease, affecting only approximately 1000 patients in the entire United States. The diagnosis requires the presence of bilateral acoustic neuromas, but many other tumors of the nervous system are also present. It is a very different disease from von Recklinghausen's neurofibromatosis, NF1. The remarkable genetic research in recent years has defined the origin of NF2 to be the lack of a specific suppressor protein, known as Merlin. While we await a method to replace this protein, the neurosurgical care of these patients is a formidable problem. The author reviews his personal series of 41 patients with NF2 treated during the past 30 years and presents 10 cases in detail to demonstrate their considerable range of differences and the treatment problems they have posed.

Key Words * neurofibromatosis type $2 *$ bilateral acoustic neurofibromatosis * central neurofibromatosis * bilateral acoustic neuromas * bilateral vestibular schwannomas * Merlin * schwannomin

This review is based on my personal series of 41 surgically treated patients with neurofibromatosis type 2 (NF2). The patients were cared for in the microsurgical era between 1970 and 1995 and received minimum follow-up care of 3 years (median 12 years). All of these people were referred because of bilateral acoustic neuromas. This was a quite young group, with the average age only 20 years, much younger than my patients with solitary acoustic neuromas. The youngest patient was 8 years old and the oldest 40 years old when first referred. Although this is not a great spread, it was sufficient to indicate strongly that growth rate and more serious multiple involvement were inversely proportional to the age and that the eventual prognosis was far worse for the children. It had become obvious during the craniectomies that the acoustic neuromas were rarely solitary intracranial tumors and were usually multiple in the cerebellopontine angle (CPA), with many small schwannomas of the other cranial nerves in addition to the acoustic neuromas. These often arose from separate fascicles of the ninth, 10th, and 11 th cranial nerves and infrequently from the facial or auditory nerves. Because they tended to be only a few millimeters in diameter, they could usually be removed by resecting only the single fascicle of origin, with preservation of neural function. I was often unable to recognize these additional small tumors on imaging studies, as they were obscured by the acoustic tumors. I was originally surprised to find that these tumors, including the acoustic neuromas, were schwannomas, not neurofibromas. This is in contrast to NF1, neurocutaneous neurofibromatosis, and von Recklinghausen's disease, in which intracranial tumors are quite rare and are most frequently optic gliomas, which are not seen in NF2. The spinal tumors of NF1 are regularly neurofibromas, as are the subcutaneous tumors. Additionally in the 
NF2 patients there were often multiple meningiomas. When operating to remove the acoustic neuroma, it was also my procedure to remove all additional tumors within the exposure that could be removed without damaging the patient.

Nevertheless I was surprised at the high incidence of serious spinal tumors in the NF2 patients. In the earlier years of this series, after an NF2 patient developed a midthoracic paraparesis a few days after removal of her first acoustic neuroma and required an emergency laminectomy for a previously asymptomatic undiagnosed T-8 meningioma, I began to study the patients for possible spinal tumors. The tumors were originally diagnosed by myelography, and then later and more easily by magnetic resonance (MR) imaging. These imaging studies were normal in only seven of the 41 patients, and in 12 patients imaging of the spine indicated that the laminectomy had to take precedence, usually causing postponement of the removal of acoustic tumors until the patient recovered from the spinal tumor removal.

Neurofibromatosis is actually two separate diseases with many features in common and many quite different. The neurocutaneous form of the disease was described by von Recklinghausen more than 100 years ago. After the distinct difference between von Recklinghausen's neurocutaneous disease and central neurofibromatosis also called BANF (bilateral acoustic neurofibromatosis) was recognized, the terms NF1 for the neurocutaneus form and NF2 for the central form were agreed upon. Neurofibromatosis type 1 is present in approximately 100,000 people in the United States and so is the most common single-gene disease. There are approximately 1000 NF2 cases in the entire United States, making NF2 fortunately quite rare. Thirty percent of cases of both NF1 and NF2 are sporadic with no family history, but then may breed true. Both are dominant autosomal genetic diseases with very high penetrance, and both appear to be single-gene diseases. Every cell in the body, both in NF1 and NF2, carries its respective abnormal gene, thus involving all three germ layers. The hallmark of both diseases is the development of tumors of Schwann cell origin: in NF1 they are neurofibromas and in NF2 they are schwannomas.

As each axon of a spinal or cranial nerves exits from the central nervous system (CNS) it is ensheathed in Schwann cells. Schwann cells are neuroectodermal cells that produce myelin, spirally wrapping it around the axons. The unmyelinated axons are within a tunnel of Schwann cells. Schwann cells produce collagen but are differentiated from mesodermal fibrocytes by the fact that they have a basement membrane. The combination of the basement membrane and the collagen that surrounds the Schwann cells is called the neurilemma which is itself surrounded by the endoneurium, where fibrocytes have been added to the collagen and basement membrane of the Schwann cells. A group of axons and their sheaths are surrounded by the perineurium to form a neural fascicle. The perineurium cells are indistinguishable from other Schwann cells. Finally, the epineurium is the duralike sheath that surrounds a nerve trunk, binding the many fascicles together.

In neurofibromas the twisted angulated long Schwann cell nuclei are scattered randomly in waves of collagen (Fig. 1 left), containing widely separated axons. They regularly involve the entire nerve. They may also be unencapsulated but demarcated extraneural masses incorporating other structures, or they may be intraneural with enlargement of every fascicle in the nerve trunk over long distances. 


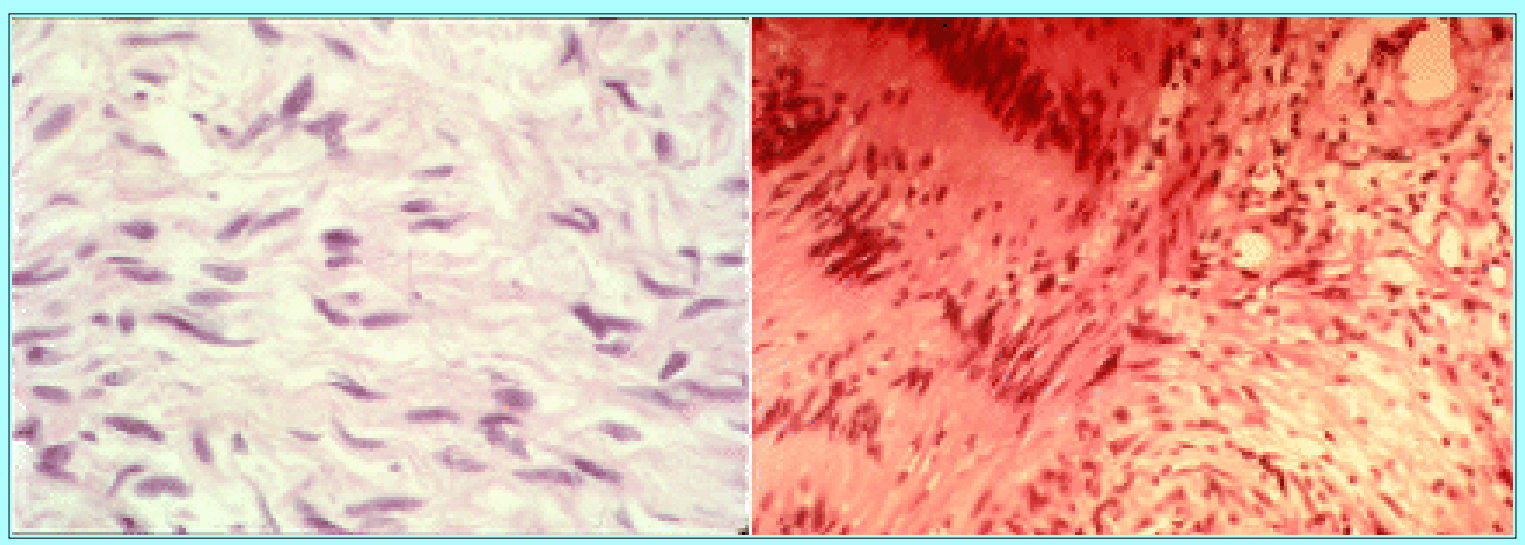

Fig. 1. Photomicrographs showing typical schwannoma cells (left) and neurofibroma cells (right).

Schwannomas generally begin in one area and grow as an expanding mass, pushing the rest of the nerve away. Histologically the nuclei of the schwannoma cells align in wavy palisades, separated by zones of fibrillary processes, known as the Antoni Type A appearance. Zones of foamlike matrix with a diffuse patternless scattering of Schwann cell nuclei are the Antoni Type B arrangement (Fig. 1 right). In solitary schwannomas, as in the usual acoustic neuroma, axons were identifed within the tumor in only a small percentage of my cases, whereas in the schwannomas of NF2 engulfed axons were not infrequent. Unlike the solitary schwannomas, not part of NF2, the schwannomas of NF2 may be multicentric with other fascicles displaced or caught between the several growth centers.

Neurofibromatosis type 1 is characterized by multiple cutaneous and subcutaneous neurofibromas, ranging from a few (five-six required for diagnosis) to an uncountable number (Fig. 2 left). Café-au-lait spots (ovoid tan skin patches) are seen, and five or so small spots or one or two of $5 \mathrm{~cm}$ are diagnostic. Lisch nodules of the iris (Fig. 2 right) can be one of the earliest signs of the disease and are pathognomonic of NF1, as are plexiform neurofibromas. The plexiform neurofibromas are candidates for sarcomatous degeneration whereas, by contrast, malignancy is not seen in NF2. In NF1 optic nerve gliomas and multiple spinal nerve neurofibromas occur, but bone tumors do not appear, although asymmetrical bone overgrowth may lead to deformity. Sphenoid wing aplasia and spinal foraminal enlargement due to dural ectasia may mimic signs of neoplasm on plain x-ray films. Acoustic neuromas or other schwannomas do not occur. A special abnormality of the renal arteries occurs in which the renal arteries are replaced by multiple very small vessels. This may produce severe hypertension that may require renal transplantation, in which case the renal artery of the new kidney will have to be anastomosed directly to the aorta. Except for the occurrence of sarcomatous change in the plexiform neurofibromas and the very rare renal artery problem, NF1 is not ordinarily a fatal disease. The NF1 gene is in region 11 of chromosome 22. 


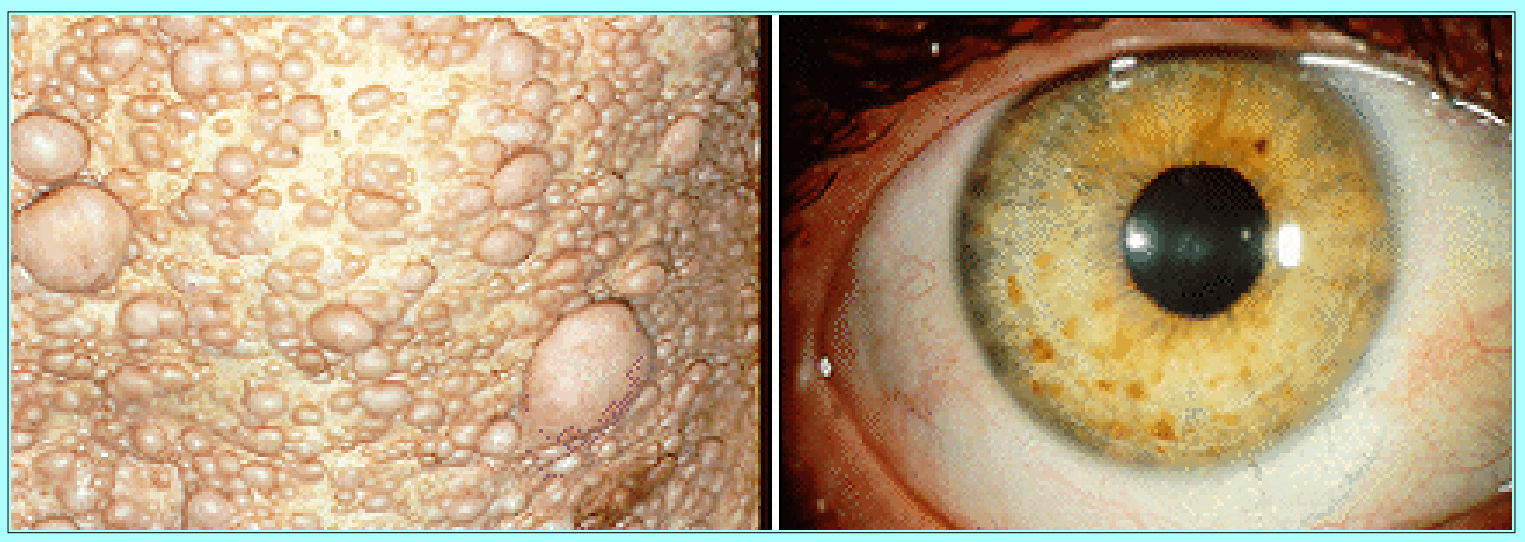

Fig. 2. Left: Photograph showing multiple cutaneous neurfibromas typical in NF1. Right:

Photograph showing lisch nodules of the iris typical in NF1.

Neurofibromatosis type 2 requires the presence of bilateral acoustic neuromas for diagnosis. These are of course vestibular schwannomas, but the term "acoustic neuroma" has been so firmly entrenched in the literature that there appears to be no point in correcting it. Actually the designation as NF2, "neurofibromatosis $2, "$ is quite inappropriate because there are no neurofibromas in NF2 and all of the neural tumors are schwannomas. In NF2, in addition to the bilateral acoustic neuromas, I have seen schwannomas of every nerve in the posterior fossa except the sixth and 12th cranial nerves. Multiple schwannomas of spinal nerves as well and cranial and spinal meningiomas are most common. There are no Lisch nodules and no renal artery changes. Cutaneous tumors are rare. Those that do occur appear to have grown from peripheral nerve sheaths and are also schwannomas, not neurofibromas. Café-au-lait spots are rare and usually too few and too small to indicate NF2. Intramedullary spinal cord tumors are frequent, usually ependymomas, although I have also encountered central schwannomas. Meningiomas are common, and every patient who has died of NF2 in my series has died as a result of overwhelmingly rapid growth of multiple meningiomas; no patient has died as a result of their acoustic tumors.

Because of the somewhat unexpected difference between the operative challenges in my patients with solitary acoustic neuromas and these patients with NF2, I decided to present clinical summaries and detailed operative reports on a number of patients who typify the range of disease and outcomes, rather than simply a statistical summary.

\section{DESCRIPTION OF SURGICAL PREPARATION}

All procedures in this group of patients undergoing suboccipital craniectomies as well as the cervical laminectomies were performed with the patient in the semisitting position (Fig. 3 upper) by using a standardized technique. Thoracic and lumbar laminectomies were performed with the patient in the 45 š prone oblique position (Fig. 3 lower). So as not to be overly redundant in reproducing this series of operative descriptions, I have selected a typical operating room preparation for a suboccipital craniectomy and will only note changes in the routine when they took place in individual cases. The patients were all admitted at least 2 days preoperatively to undergo complete evaluation and have their cases presented at staff conference. Unless emergent, the admission of any patient who had been taking aspirin was delayed for at least 1 week to allow recovery of platelet aggregation. Admission of patients who had been receiving antibiotic medications was also delayed if possible to allow for recovery of their normal bacterial flora. Antibacterial shampoo was applied the evening before surgery. 


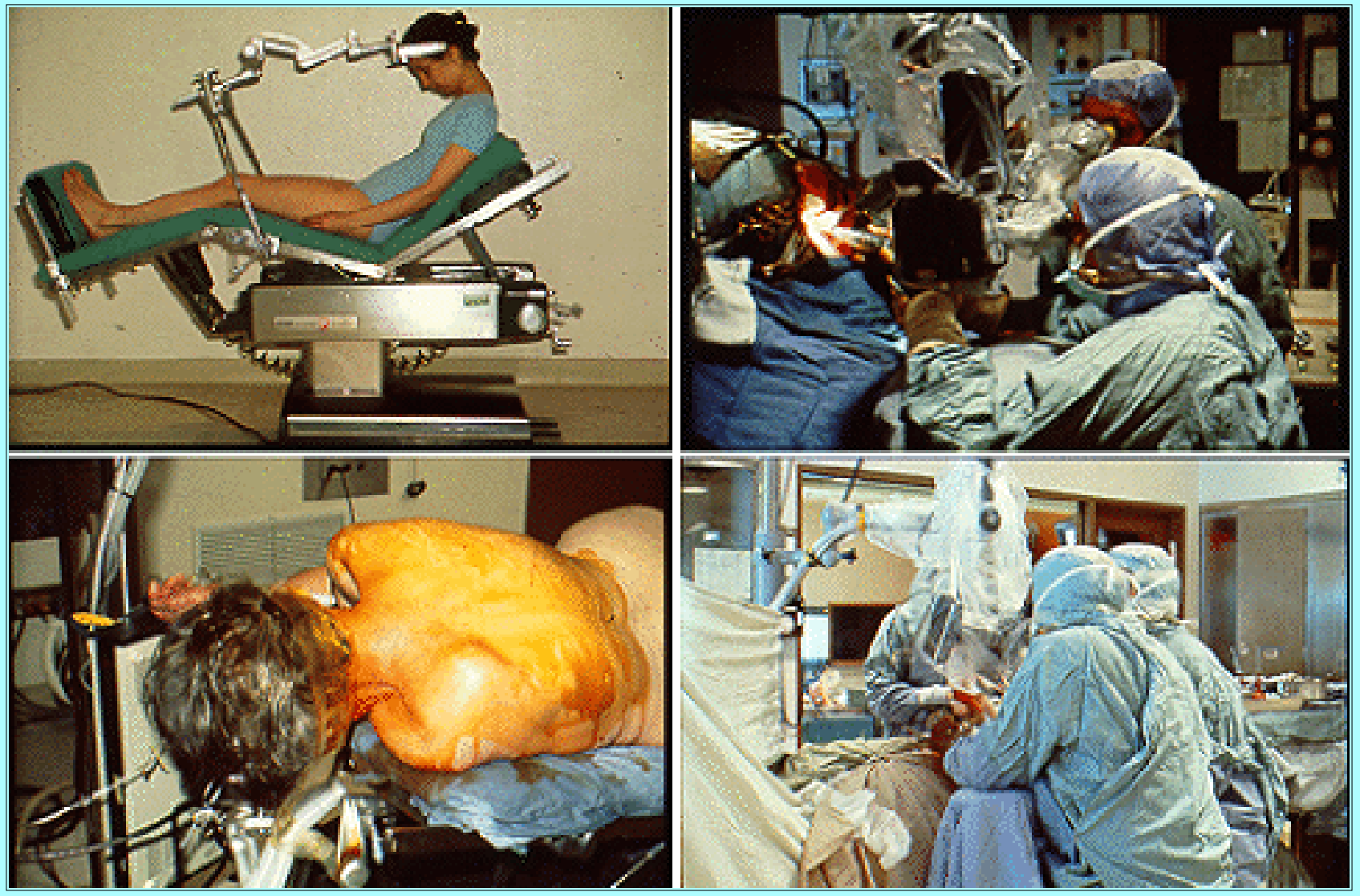

Fig. 3. Photographs showing a patient in the semisitting position (upper left) and the surgeon's position (upper right) in preparation for a suboccipital craniectomy or a cervical laminectomy. Photographs showing a patient in the 45 š prone oblique position (lower left) and the surgeon's position (lower right) in preparation for a laminectomy.

On the morning of surgery, administration of premedication was limited to atropine and a small dose of Valium. The patient was then brought to the preparation room, a room in the operating suite adjacent to the operating room, and was there placed on the operating table. Multiple intravenous lines were placed. A transvenous right atrial catheter was placed and confirmed by an x-ray study. A radial artery catheter was placed. After induction of anesthesia with pentothal (later replaced by propofol) and nitrous oxide, endotracheal intubation was performed, and muscle paralysis was produced with pancuronium. From then on the patient was artificially ventilated by the anesthesiologist to maintain desired levels of oxygen and carbon dioxide. Urethral catheterization was performed with a Foley catheter connected to a drainage bag. Intravenous vancomycin $(1 \mathrm{~g})$ was slowly infused and $80 \mathrm{mg}$ of gentamicin was injected intramuscularly. The patient was given $80 \mathrm{~g}$ of urea intravenously through the central line. I have used urea rather than mannitol throughout the years because less fluid was required, and it appeared to produce more total diuresis. The circulating thermal blanket was applied, if necessary, to correct for too much spontaneous hypothermia or hyperthermia, and the patient was wrapped in the Mast pressure suit, which was not inflated unless required during surgery. The Gardner pinned headrest was fixed in position. Somatosensory evoked potentials at the scalp were recorded from tibial nerve stimulation to measure the normal latency with the patient supine. The patient was then brought into the semisitting position with the back elevated only 30 š and the head supported in flexion without tension and without pressure with the ear-eye line downward at 10š, provided there was no change in the somatosensory evoked potential recording latency. If the latency increased, flexion was decreased until the latency returned to normal. The usual anesthesia monitoring equipment was attached, including the electrocardiograph, the arterial pressure transducer, the central venous pressure transducer, the pulse 
oximeter, the end expiratory carbon dioxide monitor, the mass spectrograph gas analyzer, and the temperature probe. A Doppler ultrasonography monitor was placed over the right atrium, the over-table instrument racks were attached, and finally, the operative area was shaved, and scrubbed with iodophor. The entire setup was then wheeled into the actual operating room, where the shaved area was rescrubbed and the patient was draped. Throughout the operation the intravenous fluid replacement was restricted sufficiently to maintain the patient at approximately $800 \mathrm{ml}$, which generally kept the serum osmolarity at approximately 130 after subtracting the allowance for the urea level. Nitroprusside was administered to keep the patient's blood pressure from exceeding a mean of $80 \mathrm{~mm} \mathrm{Hg}$. The operative procedure was then begun with the aid of 4-diameter magnifying loupes and fiberoptic headlamps.

\section{CASE 1}

History. This 35-year-old woman, an Egyptian-born engineer living in Canada, underwent the removal of several subcutaneous neuromas. There were no café-au-lait spots. She developed tinnitus and progressive deafness in the right ear, becoming totally deaf after 2 years. She presented in Canada because of headaches and staggering gait. Papilledema was noted, and a computerized tomography (CT) scan demonstrated a 4-cm acoustic neuroma on the left (the hearing ear) and a 2-cm acoustic neuroma on the deaf right side. In an effort to preserve her hearing, her neurosurgeon performed an intracapsular partial removal of the large left neuroma. The dura was left open. Although her seventh and eighth cranial nerve function was preserved, she continued to have mild papilledema, headache, and unsteadiness, with tense bulging of the craniotomy site. Repeated CT scanning showed very little change in the size of her large left acoustic neuroma.

Presentation and Operation: She was then referred and admitted to The Mount Sinai Hospital (MSH), and she underwent resection of the left acoustic neuroma. The previous craniectomy was opened, and the area of the left lateral cerebellum opening into the lateral recess of the fourth ventricle filled the field (Fig. 4 left). The craniectomy was extended laterally to expose the entire sigmoid sinus. A dural flap was formed along the medial edge of the sigmoid, and the sigmoid was drawn out over the cut mastoid surface with sutures to the muscles. This provided sufficient exposure to be able to place the flexible cerebellar retractor and support the cerebellum, permitting exposure of the tumor (Fig. 4 right).

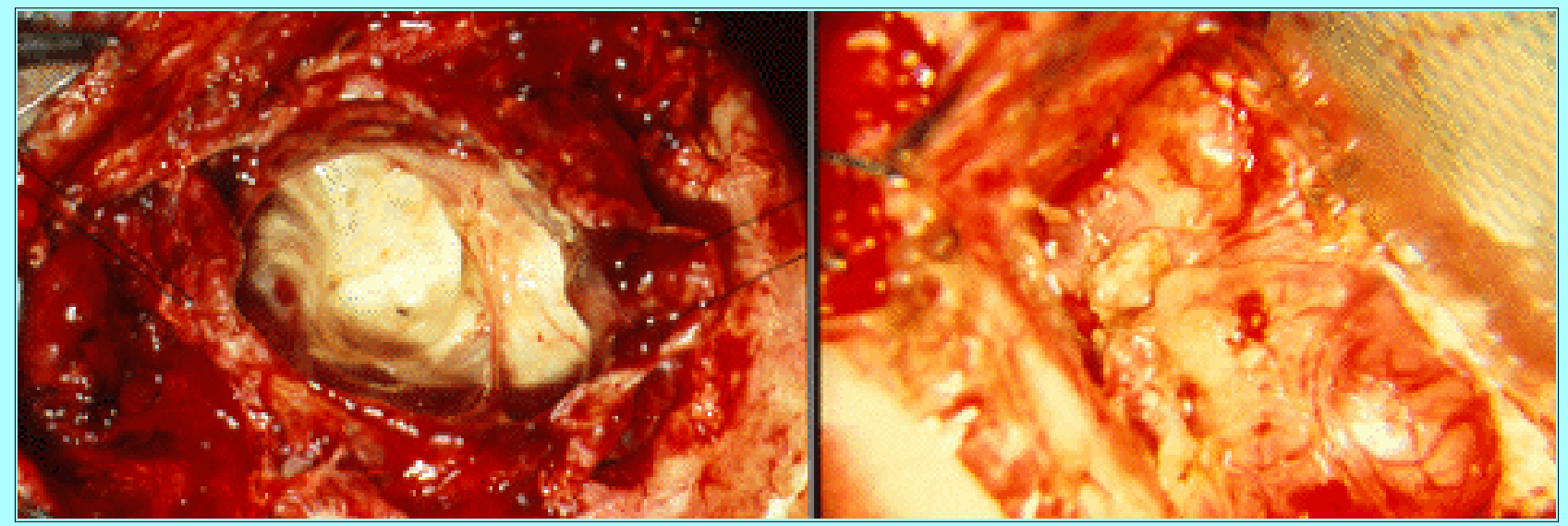

Fig. 4. Case 1. Left: Photograph taken on the patient's left side showing a secondary exposure of the operative field through the region of the previous cerebellar resection; the fourth ventricle is seen through the widely enlarged lateral recess. The photographic field is $36 \mathrm{~mm}$ in diameter. Right: Photograph showing a revision of the exposure through the more lateral exposure; tumor is seen filling the center of the photograph. The posterior edge of the tentorium is on the upper left and joins the upper margin of the posterior aspect of the 
petrous pyramid on the lower left. The flexible silicon-coated cerebellar retractor is in the right upper corner. The photographic field is $36 \mathrm{~mm}$ in diameter.

The tumor was progressively cored laterally to medially until, on reaching the medial surface, a surprisingly intact arachnoid plane appeared. This plane was preserved as the capsule was removed. The seventh cranial nerve and the auditory nerve could be seen through this membrane, although rather indistinctly because minimal blood staining of the membrane was simply controlled with cottonoid pledgets instead of removing the membrane. The fifth cranial nerve and superior cerebellar artery (SCA) had been displaced upward into the tentorial notch, whereas the anterior inferior cerebellar artery (AICA) had been displaced downward onto the ninth and 10th cranial nerves, all rather vaguely seen through the preserved arachnoid. The petrous dura posterior to the internal auditory canal (IAC) was then resected and the canal was drilled open. The tumor within the canal extended only $8 \mathrm{~mm}$; its removal, preserving the neural structures, was relatively easy. There had been no evidence of multifascicular origin or of any other tumors in the field, and the tumor appeared quite like an ordinary acoustic neuroma rather than an acoustic neuroma of NF2. It was possible to close the dura by using a sheet of gelfoam under the closure and another over the dura before the muscle closure to prevent leakage. The patient tolerated this 10-hour microsurgical procedure well and, as soon as heparin was reversed and she was extubated, she was able to hear and carry on a conversation. There was good facial function. She recovered quickly and was able to return to work as an engineer.

Subsequent Operations and Postoperative Course. She was readmitted to MSH 1 year later when the 2 -cm right acoustic neuroma in her deaf ear had grown to $2.5 \mathrm{~cm}$; it was removed uneventfully. Her facial function was preserved. Two years later severe low-back pain led us to perform another study, which showed a neuroma with a block at L-3. At laminectomy a 2-cm neuroma was resected from the cauda equina, but every nerve root of the exposed cauda was studded with tiny neuromas, looking like strings of small pearls (Fig. 5). Over the next 15 years the patient has remained well, and surprisingly, MR studies have shown no growth in any of these uncountable neuromas.

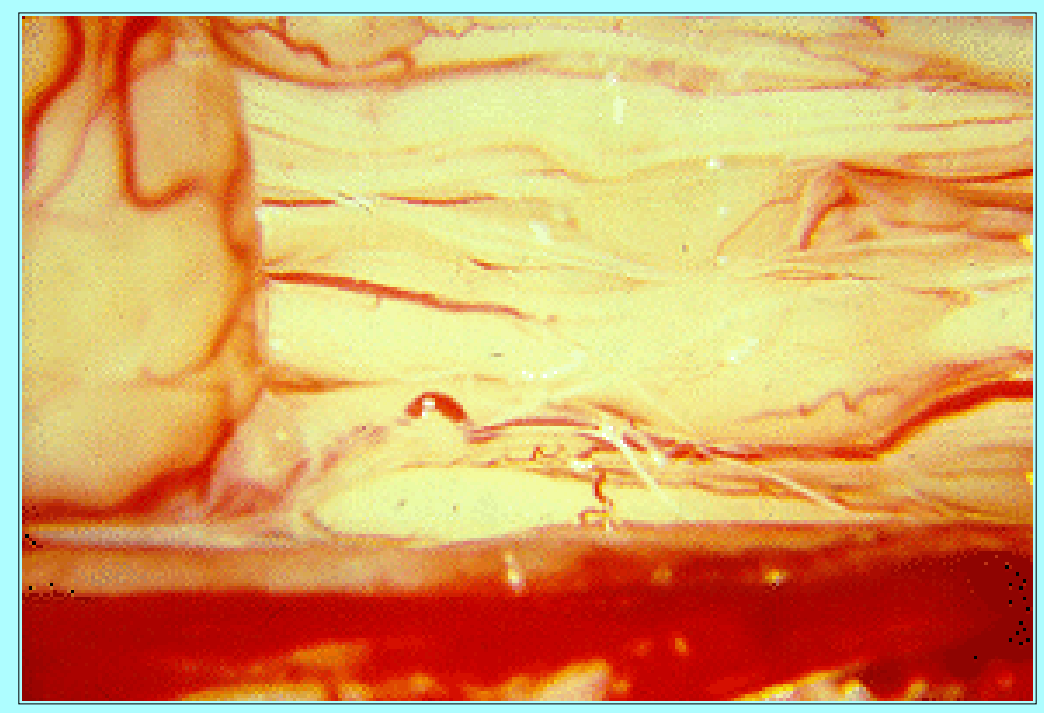

Fig. 5. Case 1. Intraoperative photograph of the cauda equina at laminectomy. A schwannoma is seen at the left edge of the photograph. The cauda roots to the right of this tumor are studded with many other schwannomas. The photographic field is $25 \mathrm{~mm}$ in diameter. 
History. This patient is a 22-year-old Caucasian woman, right handed, with NF2 diagnosed 6 years previously and with no family history of the disease. At age 15 years she underwent a craniotomy and removal of a parasagittal meningioma in Florida. She had 20/400 vision in both eyes and was considered legally blind as a result of the papilledema caused by that meningioma. She had been attending a school for the visually impared. In the past year she had become deaf on the right side and had markedly decreased hearing on the left. Bilateral acoustic neuromas were found on a scan in Florida.

Presentation. The patient was then referred and admitted to MSH with the additional history of deteriorating gait for 7 months. She had lost her ability to balance while standing still and also had general loss of coordination. Headaches with nausea and vomiting had been intermittent.

Over the few months prior to admission, the patient had lost sensation in both hands, mostly in the fingers. She had burned her left third finger but was unaware of the injury while burning it. She had bitten off a piece of her right thumb, not knowing it. She also had difficulty in fine motor movement, such as buttoning a shirt and signing her name. She noted weakness mostly in the right arm and hand. Leg strength was good. There was no incontinence.

Examination. The patient was well developed, well nourished, and awake and alert, with normal vital signs. No café-au-lait spots appeared on her skin. There were a few small fibrous nodules over the right forearm and left suboccipital area. There was a well-healed left temporoparietal scalp flap with intact bone flap beneath. The neck was supple with full range of motion and normal carotids. There was a l-cm firm nodule noted at the ramus of the left mandible, medial to the ramus. Otherwise, the general physical examination was unremarkable.

She was alert, with normal higher cortical functions. The fundi showed sharp disc margins with optic atrophy. She could count fingers with the right eye, but in the left she had only light perception. There was right abducens weakness; minimal left lateral gaze nystagmus; decreased right masticator strength; but facial sensation was normal. Facial function was symmetrical. The patient was deaf on the right side, and hearing markedly reduced on the left. The uvula rose to the left. Gag reflex was depressed bilaterally. Sternocleidomastoid and trapezius function was slightly weak, and the tongue deviated to the right. There was 4/5 strength in biceps, triceps, and deltoids, and in wrist flexion-extension and finger flexion-extension (slightly weaker on the right side). The lower extremities showed $4 / 5$ strength on the right side and $4+/ 5$ strength on the left. Sensory examination revealed decreased pin from C-4 through T-5 and position errors with the left fingers. Position sense was good in the right fingers and in the toes. Vibration was decreased below the clavicles and then to a more significant degree below C-6.

Coordination showed slight dysmetria bilaterally. Gait was slow and a cane was required for ambulation. Plantars were down going, and reflexes were normal throughout.

Neuroimaging Studies. Brainstem auditory evoked responses were consistent with CPA mass lesions bilaterally and right-sided deafness. Caloric testing revealed the absence of vestibular function bilaterally. A CT scan (Fig. 6) demonstrated three separate tumors in the left CPA with widening of the auditory meatus and two separate tumors in the right CPA, consistent with bilateral acoustic neuromas with associated meningiomas. Also noted was a left intratentorial meningioma and multiple small meningiomas about the convexity, as well as a tentorial meningioma in the area of the right lateral sinus and an en plaque tumor in the lower right temporal convexity. A myelogram with metrizamide from below, combined with CT scanning, revealed a widened cervical cord extending from the upper thoracic area with high-grade block at the C-5 level. A small neuroma was also seen. An angiogram of the spine 
showed the entire cervical cord to be filled with tumor stain. A cerebral angiogram revealed mass effect and vascularity consistent with CT findings. It was decided that the spinal tumor had to be cared for before the intracranial tumors.

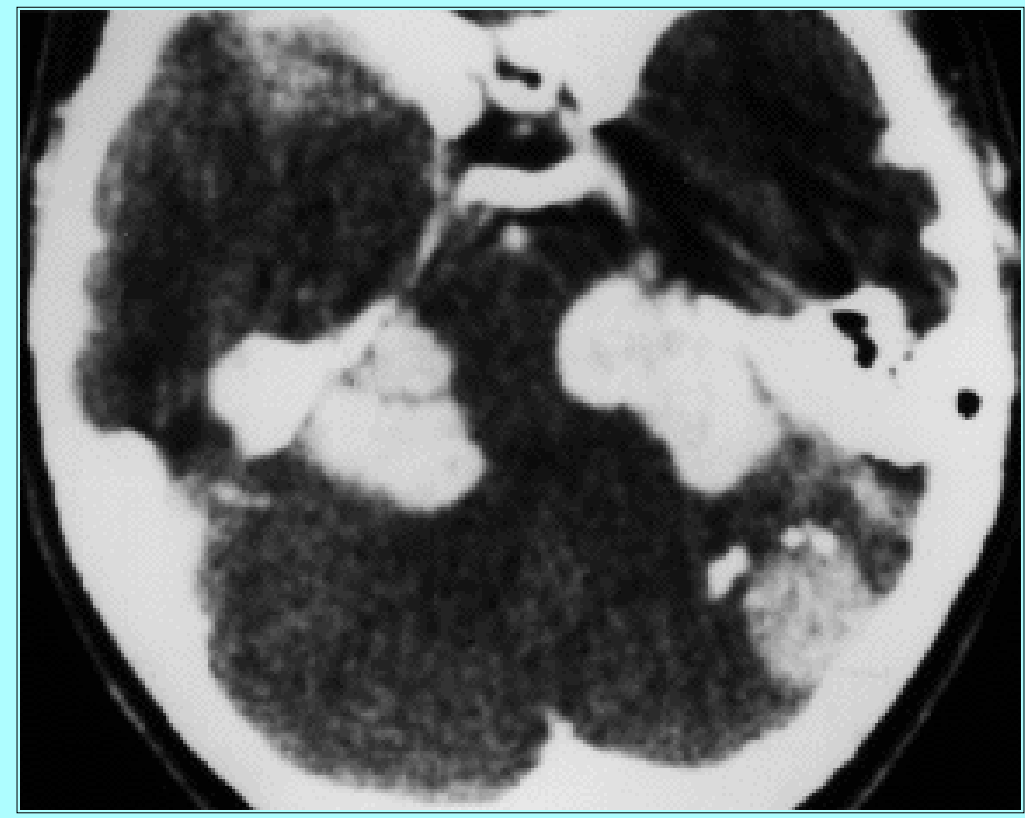

Fig. 6. Case 2. A CT scan revealing acoustic neuromas and separate meningiomas bilaterally, predominantly on the patient's left side (right side of the CT).

Operation. A laminotomy was performed on C-2 through C-7. The dura was obviously expanded; it was opened in the midline and sutured to the muscles exposing the widened cord. Under the microscope at 16 diameter magnification, the midline raphe was opened and a brown-tan vascular rather typical ependymoma spanning C2-4 (Fig. 7) was completely removed, as was a separate similar lesion extending from C4-7. Also, a small neuroma was removed from the right C-2 nerve root. This was a dorsal nerve root and only one fascicle was involved.

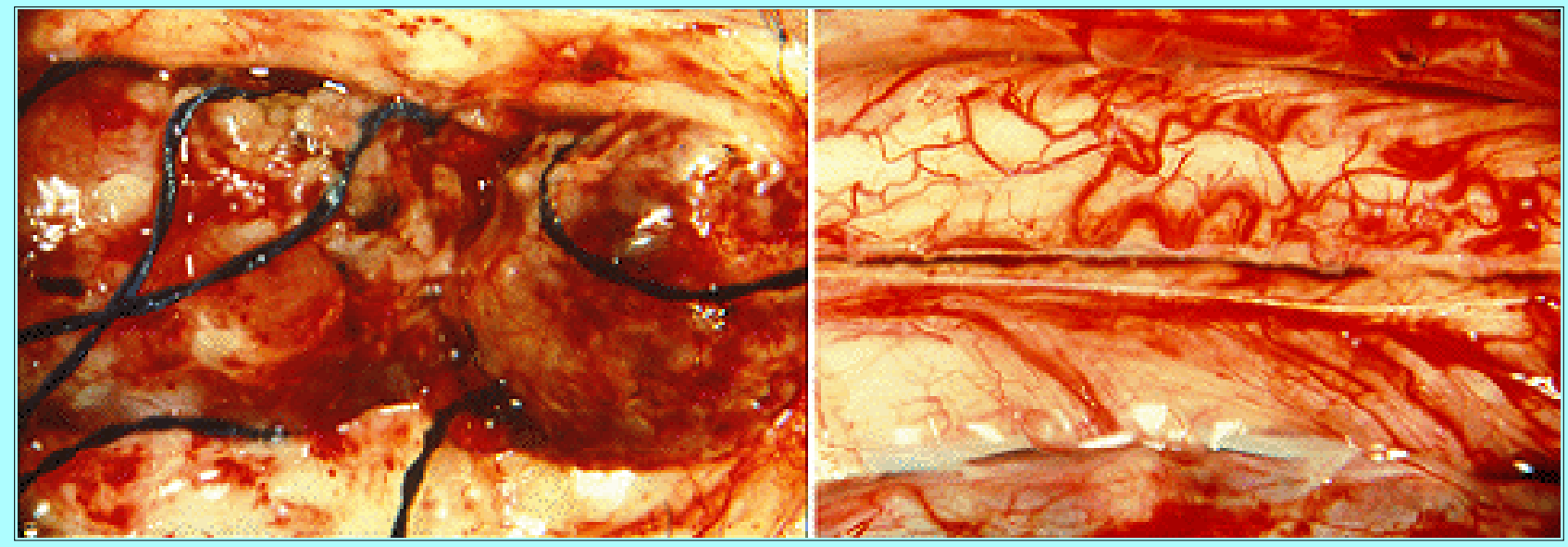

Fig. 7. Left: Case 2. Intraoperative photographs. Left: An intramedullary cervical spinal cord ependymoma is exposed through midline myelotomy, which is being held open by pial stay sutures. Right: The ependymoma has been resected and the stay sutures have been removed, allowing the myelotomy to come back together. The photographic fields are 25 $\mathrm{mm}$ in diameter.

Postoperatively, the patient did quite well with some initial increased sensory deficits with poor position 
in the toes and fingers bilaterally. Over the course of the next few days, there was rapid improvement, and the patient was soon considerably better than in her preoperative state. She was discharged to return at a later date for removal of her acoustic neuromas.

A few months later the patient was readmitted to MSH for removal of the left acoustic neuroma which was the larger of the bilateral acoustic tumors and which had increased in size since the previous imaging study. Following the usual preparation, a left suboccipital craniectomy was performed.

Second Operation. A vertical skin incision $12 \mathrm{~cm}$ long was made $2 \mathrm{~cm}$ medial to the mastoid. A subcutaneous nodule about $2 \mathrm{~cm}$ in diameter was present just medial to the incision line at about its midpoint. The skin edge was reflected from this subcutaneous neuroma, and it was resected using the bipolar coagulator to separate it. The incision was carried down through the galea, deep fascia, and muscles and the suboccipital bone and mastoid were visualized by subperiosteal dissection. The vertebral artery was visualized by sharp dissection above the lateral portion of the arch of the atlas and the Malis suboccipital retractors placed to maintain the exposure. Using the 5-mm high-speed cutting burr (Fig. 8 left), a 4-cm left suboccipital craniectomy including the entire mastoid process was performed. The descending portions of the lateral and sigmoid sinus were completely exposed (Fig. 8 right). The mastoid cells were sealed with bone wax, and the mastoid emissary vein was sealed with the bipolar coagulator. A semicircular dural opening with its base medially was made, and lateral relaxing incisions were made, one up to the transverse and one down to the sigmoid sinus. Traction sutures were used to sew the lateral dural segment to the muscles and then used to draw the lateral sinus out of the wound over the cut surface of the mastoid. The loupes and headlamps were removed, and the remainder of the operative procedure was carried out under the Zeiss operating microscope at magnifications of 10, 16, and 25 diameters.
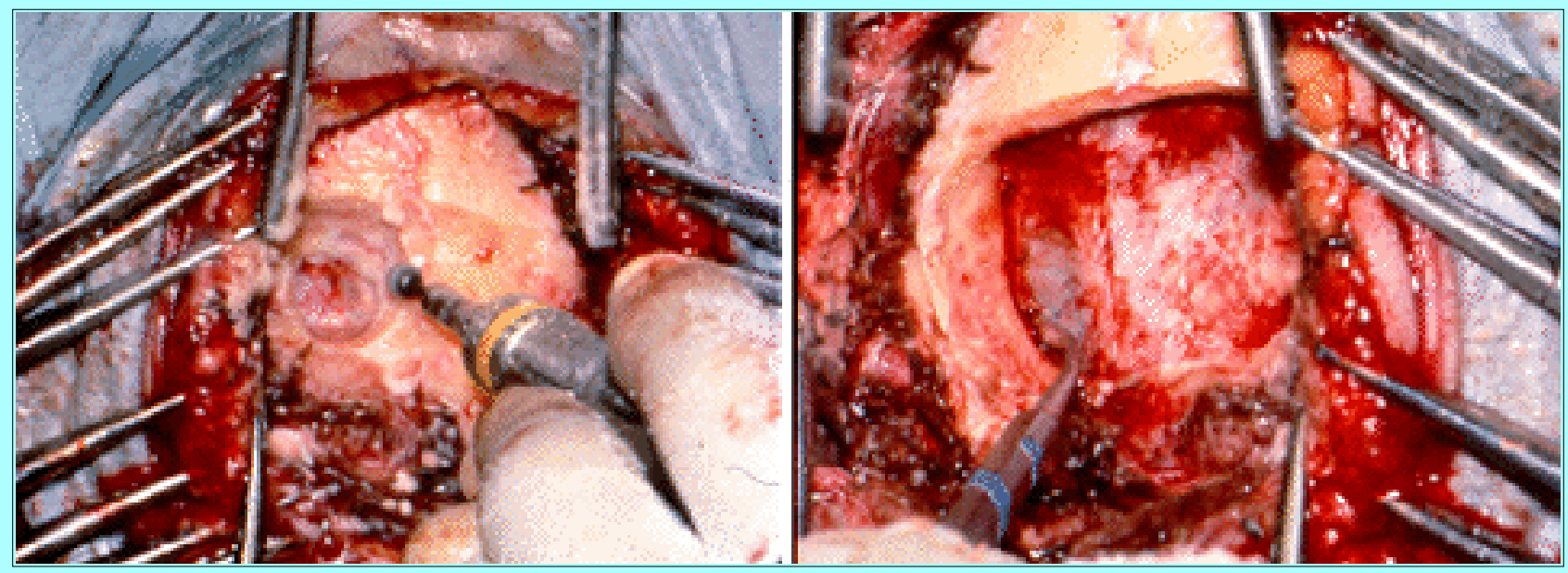

Fig. 8. Intraoperative photographs showing a left suboccipital craniectomy in which the bone is being removed by using a 5-mm high-speed burrhole drill (left). The sigmoid sinus has been completely exposed and the craniectomy is continued to the flat of the petrous bone. The photograph fields are $15 \mathrm{~cm}$ in diameter.

The posterior fossa, after exposure of the dural sinuses, could be seen to be somewhat higher vertically than usual, although perhaps a bit narrower in its lateral diameter. Along the sigmoid sinus, the firmness of an intradural meningioma could be felt at that point, and there was clearly an intradural abnormality about halfway between the lateral margin of the lateral sinus and the torcular Herophili. Widening of the bony opening upward and a little more medially superiorly was carried out across this part of the sinus to create room to approach this area. The dura was now opened with a horseshoe-shaped flap with its base 
medially. As the upper edge was visualized, the margin of a meningioma coming from the tentorium and extending down into the cerebellum was immediately visualized (Fig. 9 left), and laterally a separate meningioma was present on the descending portion of the sigmoid sinus (Fig. 9 right). Dural stay sutures were now placed to hold dural retraction.

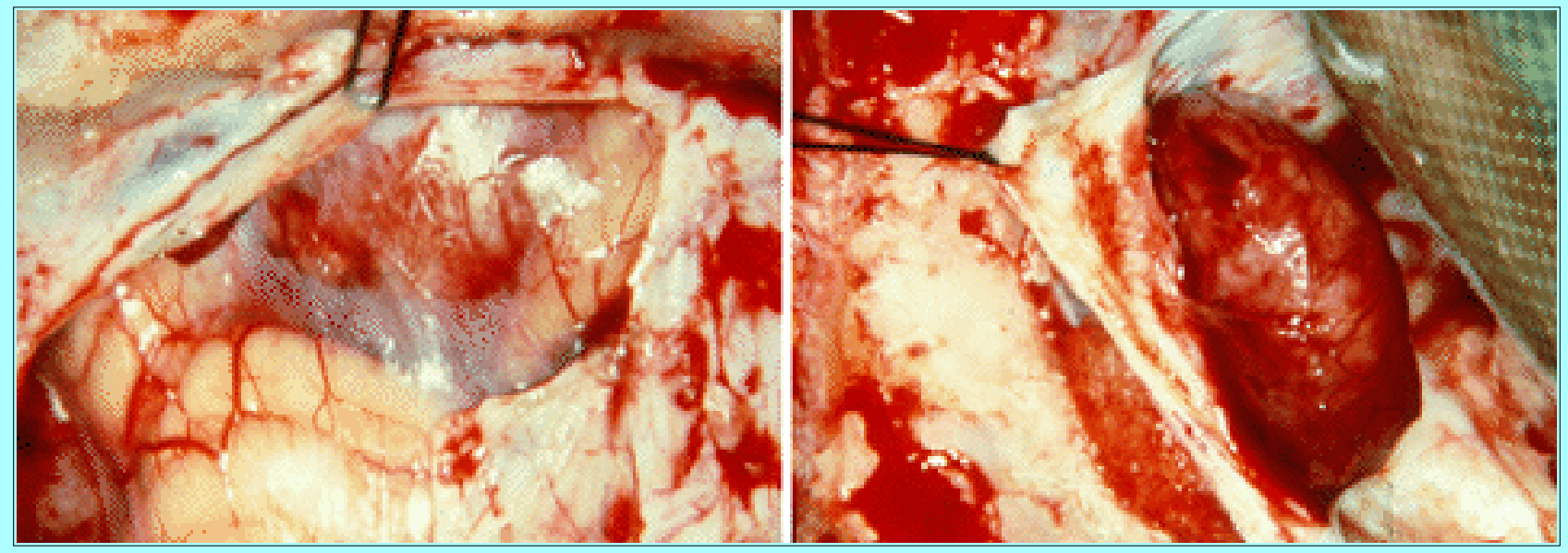

Fig. 9. Case 2. Intraoperative photographs showing a tentorial meningioma arising just anterior to the lateral sinus (left) and the posterior surface of the meningioma arising from the petrous dura just anterior to the sigmoid sinus (right). The photographic fields are 36 $\mathrm{mm}$ in diameter.

Dissection was begun about the meningioma superiorly and posteriorly on the tentorium. The plane of cleavage to the cerebellum was visualized by cutting the arachnoidal attachments and progressively separating the arachnoid and the pia of the cerebellum around enough of the tumor to begin to core it upward. Coring was carried out with the bipolar cutting current. The tumor itself was a bit unusual. It was whitish, yellowish, but nevertheless dural in origin yet would have been difficult to distinguish from a fibrous neurofibroma; however, it proved to be a meningioma. It measured approximately $2.5 \mathrm{~cm}$ in diameter. After it was cored significantly, the margins could be brought in, cerebellar separation completed and protected with cottonoids, and the attachment to the tentorium resected. Here, the tumor separated the tentorium into two quite separate leaves, pushing the superior surface of the tentorium upward and going between the two leaves, thickening the tentorium. The lower surface of the tentorium was now resected and the tumor removed from the dome of bulging upper leaf widely around. The occipital arachnoid was not seen because the thin upper layer of the tentorium was left in situ. Blood supply to this tumor came through a tentorial artery. Hemostasis was completed with the bipolar coagulator. A small strip of Surgicel was placed in the margin of the tentorial partial resection.

The Malis self-retaining cerebellar retractor was now placed and used to begin to elevate the cerebellum in the plane of the petrous. This gave enough room, after less than a centimeter of separation, to free the arachnoid along the cerebellum and to demonstrate that the meningioma arising from the posterior petrous extended down under its own arachnoid toward the foramen magnum and up toward the tentorium. Using a bit of lift on the lower cerebellum, it was possible to open the arachnoid behind the 11 th cranial nerve at the foramen magnum rim and achieve free flow of cerebrospinal fluid (CSF). Now the retractor could be placed to visualize more adequately visualize the meningioma along the sigmoid sinus. This was a typical meningioma, purplish and quite vascular. Its attachment was to the dura of the posterior petrous coming almost to the sigmoid sinus and then lying on the sigmoid but not actually infiltrating it. It was possible to get a plane of cleavage on the dura and to totally resect this tumor in one piece by melting its arachnoid attachments and freeing it from the dura with the bipolar coagulator. The 
blood supply of this tumor came from the dura of the petrous. The tumor measured approximately 1.5 to $2 \mathrm{~cm}$ in diameter and $2.5 \mathrm{~cm}$ in length.

The self-retaining retractor could now be further adjusted as the arachnoid was dissected free to allow the elevation of the cerebellum to a distance of $1.5 \mathrm{~cm}$ in the plane of the petrous pyramid. With this separation, it was possible to incise the capsule of the large acoustic tumor and to begin its coring. The tumor was vascular on the surface as well as within but was tough, fibrous, whitish-pink, and much firmer than most acoustic neuromas with fibrous zones similar to a meningioma. Coring was carried out with the bipolar coagulator cutting mode to resect segments of the tumor. After significant coring, it was possible to begin elevating the tumor in order to visualize its lower pole. As this was carried out, the 11th, 10th, and ninth cranial nerves were separated progressively from the undersurface of a double arachnoid layer, and the dissection was carried back medially with a partial posterior-inferior cerebellar artery (PICA) loop of modest size adherent to the capsule. The posterior-inferior loop was preserved while the branch to the tumor itself was coagulated and cut, and the nerve separation was carried back to the posterior and inferior displaced flocculus and the choroid plexus. It was clear that the ninth, 10th and 11th cranial nerves each had beaded zones of neuromas within them (Fig. 10) obviously not involving just single fibers, although some of the fibers appeared clear, but with a multiplicity that precluded removal without interference with function. The flocculus was herniated down into the foramen magnum and the 10th canial nerve and that portion of the medulla was in part within the foramen magnum. The tumor extended down onto the vertebral artery lying on the area in which the PICA originated from the vertebral artery and was crossed by three fascicles of the 12th cranial nerve. These fascicles were free of tumor insofar as they could be seen. The tumor was separated from this area after coring it more precisely, and a strip of glove rubber was placed over the nerves from ninth through the 12th cranial nerves and over the PICA and vertebral artery.

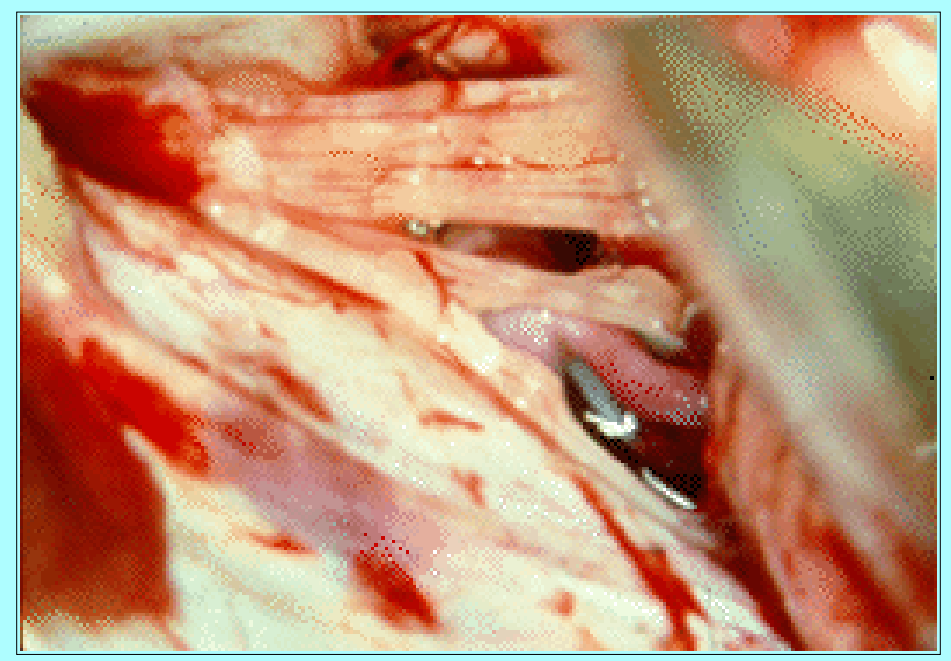

Fig. 10. Case 2. Intraoperative photograph showing that the ninth, 10th, and 11th cranial nerves entering the jugular foramen are studded with small schwannomas. The posterior inferior cerebellar artery can be seen through the space between the 10th and 11th nerves. The photograph field is $25 \mathrm{~mm}$ in diameter.

Upward coring of the tumor was continued. The vestibular nerve complex entered directly into the tumor, whereas the auditory and facial nerves were adherent to the tumor capsule anteriorly to the choroid plexus and were significantly thickened. For the time being, they were left intact and dissection was carried upward along the brainstem beneath the fold of the cerebellum and upward along the tentorium. The vein of the lateral recess and the petrosal vein were tightly stretched over the capsule, 
restricting the medial separation of the cerebellum at a point at which the tumor extended deeply medially. These veins were sealed with the bipolar coagulator and divided.

Extending the dissection further upward, the tumor was separated from the fourth cranial nerve just past the tentorial notch and brought down along the fifth cranial nerve fibers and the SCA. It could now be seen that there were two major clefts within the tumor running in a medial-lateral direction and that the tumor was composed of at least three separate partly fused lesions with some neural structures lying in the clefts between and then being fused into the tumor where the clefts sealed. The tumor was, nevertheless, brought down from the tremendously distorted fifth cranial nerve, which was then followed backward from where it had been displaced through the notch to where it entered Meckel's cave. After this portion of the tumor had been resected, it was obvious that there was a separate nodule lying within the fifth cranial nerve bundle and covered posteriorly by fibers of the fifth cranial nerve through which the nodule could be seen. This was about $1.5 \mathrm{~cm}$ in diameter and was clearly a separate fifth cranial nerve neuroma (Fig. 11). This tumor was not removed because it clearly went into Meckel's cave and the need for at least temporary preservation of corneal sensation was considered absolute.

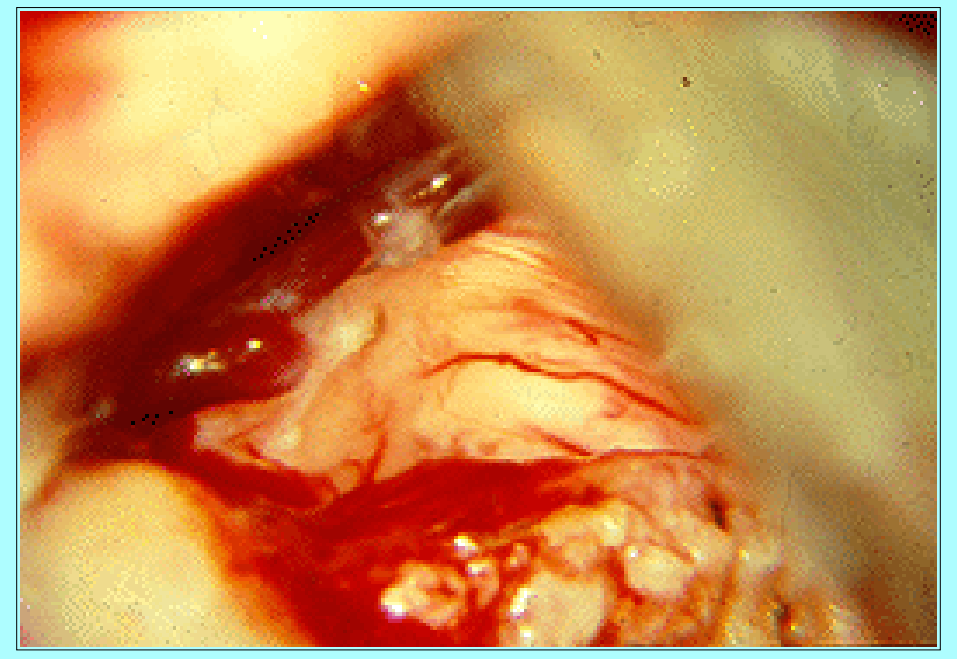

Fig. 11. Case 2. Intraoperative photograph showing that the upper surface of the cored acoustic neuroma has been dissected from the fifth cranial nerve, which contains a separate neuroma. The photographic field is $25 \mathrm{~mm}$ in diameter.

The dissection was now returned to the inferior medial margin of the tumor and carried forward so that the facial nerve was separated as much as possible, although it clearly had nodular enlargement to the point at which it attenuated into the capsule to, perhaps, one-third its normal size, about $1.5 \mathrm{~cm}$ anterior to the choroid plexus. More anteriorly, the nerve widened out again and the main trunk of the AICA was visualized coming up from the basilar artery (BA) medial to the sixth cranial nerve, which was adherent to the tumor. The sixth cranial nerve was liberated by arachnoidal dissection and was free of any tumor involvement. Three or four branches extending from the AICA loop into the tumor were sealed with the bipolar coagulator and cut, and the main trunk was turned backward to the brainstem without damage. Carrying the dissection the rest of the way around, the facial nerve was reached again and followed upward, beneath the curve of the fifth cranial nerve, and then turned backward to enter the area anterior to the ICA, at this point coursing posteriorly.

Completion of removal of the major remaining medial mass of this tough, fibrous tumor was now carried out leaving only a segment protruding about $0.5 \mathrm{~cm}$ from the widened internal auditory meatus (IAM). The removed tumor measured $4 \mathrm{~cm}$ in diameter. The petrous dura was now incised in a rectangular 
fashion from the IAC posteriorly to reach almost to the sigmoid sinus, and this segment of dura was resected. It was covered with psammomatous infiltrations and had one small meningioma on it as well that was resected. Now the posterior wall of the bony canal was drilled away. The canal was widely funneled by the tumor mass within it, measuring about $12 \mathrm{~mm}$ in height and was eroded past the crista with a vestibular opening into which the tumor protruded inferiorly and posteriorly to the crista. After drilling out of the canal, the opening of the dura of the canal demonstrated that the neural structures within the canal had been essentially replaced by three separate tumors. The inferior vestibular nerve could be followed as a tumorous mass coming from one of the previous lobules of the major angle lesion and extended with tumor tissue in it directly into the vestibule; there, by traction backward, it was possible to resect the tumor from within the vestibule. Much larger was the superior vestibular portion of the tumor that was nearly a centimeter in diameter intracanalicularly and readily cleaved from the inferior vestibular and from the structures just anterior to it. This portion of tumor also came from the main tumor fusion group and went out into the superior vestibular canal above the somewhat eroded crista.

After this was resected, it could be seen that the auditory nerve itself was totally involved with tumor as a spindle taper on the end of a carrotlike mass that came down anteriorly beneath the crista to end in more normal-looking neural fibers that came directly from within this nodule of the tumor just at the entrance to the cochlea (Fig. 12). Dissection backward demonstrated that it was not possible to preserve any of this neural structure and that medially there had been no evidence of continuation of the auditory nerve through the tumor. With the resection of the remaining portion of the tumor and the auditory nerve, the facial nerve could be followed to the entrance to the facial canal as it crossed diagonally upward from beneath the auditory portion of the tumor. However, as previously noted, just anterior to the flocculus, the facial nerve had been partly replaced by tumor so that less than one-third of its substance was still present, and this nerve was attenuated and markedly altered in appearance, although still continuous. It is quite possible that what appeared to be continuity represented only sheath and arachnoid layers, with no actual functional or neural tissue. However, because there was no residual tumor remaining, the facial nerve was left in this manner with a segment about $5.5 \mathrm{~cm}$ long, running from the pons forward and downward, greatly attenuated, and then thickening up; curving below the fifth cranial nerve and then turning more laterally and backward; and entering the canal at its anterior-inferior margin, running lateralward in the canal, and then curving sharply upward to enter the facial canal.

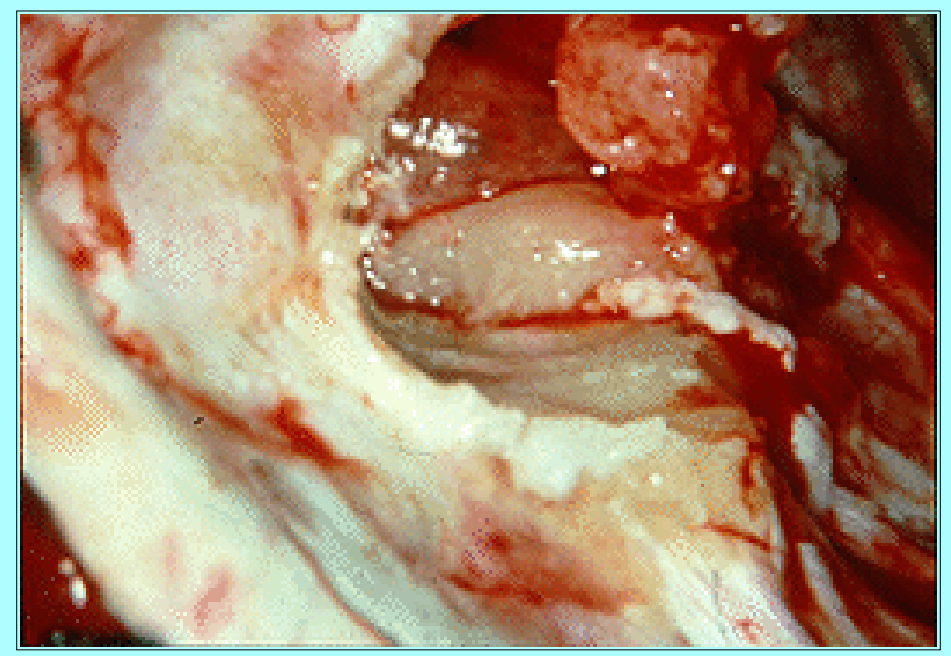

Fig. 12. Case 2. Intraoperative photograph revealing that the posterior wall of the internal auditory canal has been drilled away and the tumor, which was filled with vestibular nerves 
has been resected. The auditory nerve is seen becoming a separate spindle-shaped nodule within the canal. The photograph field is $25 \mathrm{~mm}$ in diameter.

This completed the total removal of the acoustic tumor and two large and one small meningiomas. Multiple microneuromas, averaging 2 to $3 \mathrm{~mm}$ in diameter, were left in situ in the ninth, 10th, and 11th cranial nerves, and a l-cm neuroma was left in situ in the fifth cranial nerve. The sixth and 12th cranial nerves appeared free of tumor. All of the arteries from the SCA down through the PICA and the vertebral artery had been dissected free and were well preserved. The brainstem, despite its tremendous distortion and compression, appeared to be quite normal, with normal vascularity. The petrosal vein and the vein of the lateral recess had been resected. The lateral and sigmoid sinus had been preserved despite the resection of the meningiomas that had been attached just anterior to them.

A segment of subcutaneous fat was cut from the neck incision, and small fat nodules were pressed into the opening of the vestibule and into the mastoid cells, which had been drilled out in the canal. A segment of fat the size of the drilled area was placed to fill the drilled canal and sewn in place to the dural margins with No. 6-0 monofilament sutures. The self-retaining cerebellar retractor was removed, and the cerebellum, despite the deep cups where the two meningiomas had been removed, was noted to be intact and untraumatized. One SCA draining vein was under tension due to the dropping down of the cerebellar hemisphere into the relatively large remaining cavity in which the angle tumor had been, and this vein was coagulated and sealed with the bipolar coagulator in order to prevent the possibility of late bleeding.

Dural closure was now carried out with No. 4-0 Nurolon sutures. A Hemovac drain was brought through a separate posterior stab wound down to the dural surface, and the dura was covered with a sheet of gelfoam. The muscle layers and deep fascia were closed with multiple rows of interrupted No. 2-0 Nurolon, and the galea and subcuticular layers were closed with inverted No. 3-0 Vicryl interrupted sutures. The skin was closed with interrupted sutures of No. 4-0 Nurolon. The patient tolerated this 12-hour microsurgical procedure well and was returned to the intensive care unit (ICU) already recovering well from anesthesia. Her facial function remained adequate, although, as expected, the hearing in this greatly impaired left ear had been lost and she was now totally deaf.

Third Operation. One month later the patient was readmitted for removal of the right acoustic neuroma. After the usual preparation, a 4-cm suboccipital craniectomy including the entire mastoid process was performed. The descending portion of the lateral sinus and sigmoid sinus were completely exposed. The mastoid cells were sealed with bone wax, and the mastoid emissary vein was sealed with the bipolar coagulator and covered with gelfoam. A semicircular dural opening with its base medially was made, and lateral relaxing incisions were made, one up to the transverse and one down to the sigmoid sinus. Traction sutures were used to sew the lateral dural segment of the muscles and then used to draw the lateral sinus out of the wound over the cut surface of the mastoid. The loupes and headlamps were removed, and the remainder of the operative procedure was carried out under the Zeiss operating microscope at magnifications of 10, 16, and 25 diameters. The dura had been under moderate tension, and after its incision, the arachnoid was opened along the 11th cranial nerve and the flow of CSF allowed the beginning of the arachnoid dissection that required approximately a centimeter of working space. The Malis self-retracting brain retractor was placed and used to elevate the cerebellum in the plane of the petrous pyramid. As the arachnoid was dissected free along the cerebellum and down along the 11th cranial nerve, the fact that the ninth, 10th, and 11th cranial nerves were studded with small neuromas became obvious. 
There were four neuromas among the fascicles of the ninth cranial nerve, each just a couple of millimeters in diameter. Two of them were separated because they clearly could be removed without damaging the rest of the fascicles. The other two were considered too small to remove and so intimately involved with the fascicles that ninth cranial nerve damage would have occurred. A considerably larger tumor about $7 \mathrm{~mm}$ in diameter was incorporated among the fascicles of the 10th cranial nerve, extending anteriorly (Fig. 13 left). One small fascicle of the 10th cranial nerve was entirely engulfed, and the tumor was resected in one piece with this fascicle, protecting the rest of the 10th cranial nerve (Fig. 13 right). Two additional small tumors were visualized among the fascicles of the 11th cranial nerve: one of these was basketed by the fibrils and could be removed without injury to the fascicles; the other one totally invaded the fascicle, was only about $2 \mathrm{~mm}$ in diameter, and was left in place.

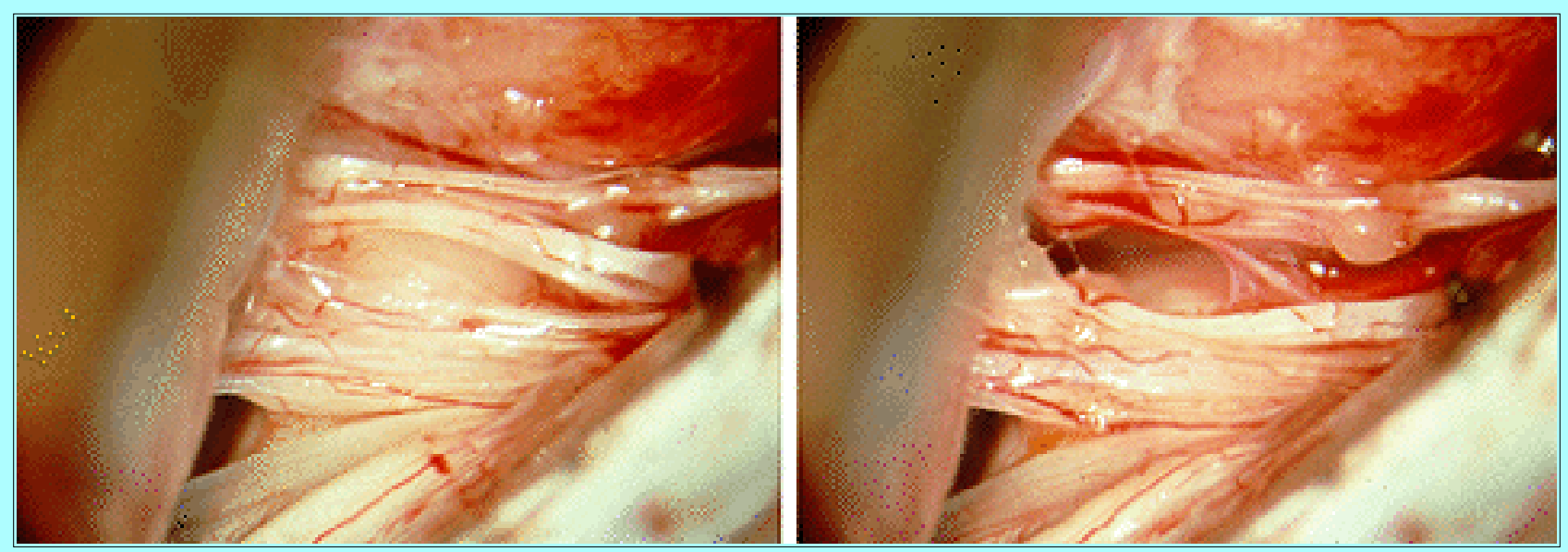

Fig. 13. Case 2. Intraoperative photograph taken on the patient's right showing the retractor on the left side. The lower edge of the acoustic neuroma is in the right upper corner. There is an oval schwannoma extending anteriorly between the ninth and 10th cranial nerves. The ninth, 10th, and 11th nerves are studded with small tumors (left) and the 10th nerve tumor has been resected along with its one fascicle of origin (right). The photographic field is 25 $\mathrm{mm}$ in diameter.

Lateral and posterior to the IAC, a meningioma about $8 \mathrm{~mm}$ in diameter stretched from the dura up to the tentorial surface. This meningioma was totally resected using the bipolar coagulator and bipolar cutting to resect the dural attachment. The arachnoid dissection was carried further, and the retraction could now be advanced to $1.5 \mathrm{~cm}$, which allowed visualization of the posterior surface of the main acoustic neuroma. It was immediately obvious that this tumor was made up of a number of separate tumors closely attached. As was demonstrated progressively, there were four tumors, of which three were rather large and one small, all as separate nodules with planes between them as well as arachnoid layers, neural fibers, and vascular channels, as well as capsular layers. Each of these nodules was removed separately and cored separately in order to free any neural and vascular trunks in the planes between them. The coring was carried out in the usual manner using the bipolar coagulator to necrose small bits of the tumor that could then be sharply removed and also using the bipolar cutting to remove small tumor blocks under continuous irrigation.

Taking down the superior nodule of the tumor, it was noted that the posterior cerebral artery, the SCA and the fourth cranial nerve had been displaced upward through the tentorial notch. This nodule had pushed the fifth cranial nerve downward and extended over it through the notch. As this portion of the tumor and capsule was resected, the fifth cranial nerve was freed and it could be seen that at the root entry zone of the fifth cranial nerve there was a separate neuroma approximately $4 \mathrm{~mm}$ in diameter. 
Another major nodule of the acoustic neuroma was just inferior to this tumor, pushing medially and going anteriorly beneath the fifth cranial nerve and indenting the brainstem. This nodule was also resected separately and brought down, freeing the fifth cranial nerve from beneath. The fibrils of the fifth cranial nerve were separated and the fifth cranial nerve tumor was resected with the sacrifice of only one tiny fibril. However, other fibrils of this nerve had little nodular bulges within them presaging future problems. The lowest two nodules of the acoustic neuroma occupied the inferomedial and inferolateral part of the major mass with the facial nerve medial to this inferior pair of tumor masses and then the facial nerve curved around anteroinferiorly and then came up to the IAC. The auditory fibers were splayed along the inferior surface of these nodules, and the auditory fibers then entered the tumor just medial to the IAC. These two tumor nodules were then resected after coring in the same manner, separating the AICA, which had only a slight lateral loop on the capsule, and preserving the facial and auditory fibers. A tuft of tumor, into which the auditory nerve entered, remained, extending out of the IAC. The auditory nerve looked reasonably good up to the point at which it entered the tumor, but the facial nerve had at least two small neuromas within its fibers. These were removed by sharp dissection with preservation of most of the facial fibers.

The dura of the petrous pyramid was then resected as a rectangular segment including the posterior margin of the auditory canal and extending backward almost to the sigmoid. Through this dural opening, using the high-speed nitrogen-powered turbine drill, the posterior wall of the IAC was drilled away. The canal was ballooned with a greater widening, about $5 \mathrm{~mm}$ from the meatus than at the meatal rim so that the total drilling height at the middle of the canal was about $10 \mathrm{~mm}$, whereas at the medial end it was 8 $\mathrm{mm}$, and laterally it was $6 \mathrm{~mm}$. The total transverse diameter of the drilled area was approximately 12 $\mathrm{mm}$. After the dura of the canal was opened, it could be seen that the entire canal was filled with tumor. This portion of the tumor was cored out in order to permit its separation from the underlying neural fibers. The facial nerve trunk was able to be separated coming from the entrance to the facial canal above the crista anteriorly, and the facial nerve lay against the anterior wall of the canal and then crossed its rim and came downward going back to the brainstem. The auditory nerve fibers were entirely engulfed within the tumor nodule, and the auditory and vestibular fibers were completely part of the tumor and, thus, no identification or separation of these fibers could be achieved within the canal. This situation had been relatively expected because the patient was totally deaf in this ear preoperatively. The tumor within the canal had been completely resected, sparing the facial nerve fibrils. The total size of the intracranial portion of the conjoined tumor nodules had been about $3.5 \times 4 \mathrm{~cm}$. Total removal had been achieved, although there were still tiny nodules in the ninth cranial nerve trunks that had not been resected. All the vascular structures, including the AICA, PICA, and SCA had been well preserved. The fourth and sixth cranial nerves were intact and looked quite normal. The fifth cranial nerve appeared to have been well maintained in its integrity, despite the removal of the tumor nodule from within it, and this was also true of the 10th and 11th cranial nerves. The ninth cranial nerve had also been well preserved, although there were still several tiny nodules within it. The seventh cranial nerve was anatomically present, although it had received a great deal of dissection when freeing it from the major tumor and when resecting the two small tumors that were within it. There had been a total resection of the auditory and vestibular nerve fibers.

A segment of subcutaneous fat was now removed from the lower end of the incision and used to fill the drilled-out space in the petrous pyramid and auditory canal and was sutured into place with No. 6-0 Nurolon sutures. The dura was closed with No. 4-0 Nurolon sutures and then a sheet of gelfoam was used to cover the closed dura. A Hemovac drain was brought in through a separate posterior stab wound and 
carried down to the epidural space. The deep muscles and fascia were closed with multiple anatomical rows of No. 2-0 and No. 3-0 interrupted Nurolon; the galea and subcuticular layers were closed with No. 3-0 inverted Vicryl; and the skin was closed with a separate layer of No. 4-0 interrupted Nurolon. Blood loss was estimated at just about $400 \mathrm{ml}$ and was not replaced. The patient tolerated this 9 hour and 30-minute microsurgical procedure well and was sent to the ICU in good condition.

Postoperative Course. In order to help her deal with her visual deficit, I set up and gave her a television set with a television camera mounted over a lighted book holder, which provided onscreen magnification of X 10. With this arrangement she was even able to read the newspaper, at least with her right eye. Despite her dreadful combination of severe visual handicap and deafness, this remarkable young woman has remained cheerful, cooperative, and optimistic. Through the more than 10 years of follow up to date, none of her myriad other tumors have progressed. She finished her schooling and has been employed within the supportive environment of the school.

\section{CASE 3}

History. This patient was a 14-year-old Caucasian right-handed woman with a diagnosis NF2, with no family history of the disease. She reported a history of headaches and subsequent physical examination showed papilledema. Her CT scan was read as showing a parietooccipital mass of approximately $6 \mathrm{~cm}$, and she underwent a right parietooccipital craniotomy with removal of angioblastic meningioma. The patient underwent a reexploration 1 month later for residual tumor, but none was found at that time. Follow-up CT scanning 9 months later revealed bilateral acoustic neuromas, not previously seen, with the left larger than right $(3 \mathrm{X} 4 \mathrm{~cm})$. An audiogram demonstrated complete loss of hearing in the left ear, although this was unknown to the patient at that time (she answered the phone using her right ear). The following month a suboccipital craniectomy was performed with total removal of a large left-sided acoustic neuroma. The outer third of the cerebellum and the eighth cranial nerve were taken at this time. She had a severe left seventh cranial nerve deficit. Postoperatively the patient did well, except for a mild gait disturbance. Follow-up CT scans since the time of that procedure had shown growth of the right acoustic neuroma, previously $1.3 \mathrm{~cm}$ and increasing to $2 \mathrm{~cm}$ by the next year, with no recurrence on the left. There also was a long history of left-sided foot drop, but previous myelograms had shown no abnormalities, and the cause of this foot drop was unknown. All of these procedures had been carried out in Colorado.

Presentation and Neuroimaging Studies. She was then referred to me for further care. On her first admission to MSH her physical examination was notable for demonstrating a partial left seventh, complete left eighth, and a complete left foot drop. Rapid alternating movements on the left were decreased. Angiography showed a right acoustic neuroma with evidence of stain and marked elevation of the petrosal vein, which was draped over the superior aspect of the lesion. There were postoperative changes noted in the right carotid distribution. Myelography demonstrated complete block from below at the level of T- 8 by an intradural extramedullary mass located on the right side of the theca. Multiple smaller lesions at the levels of T-9 and T-11 were also seen (Fig. 14). 


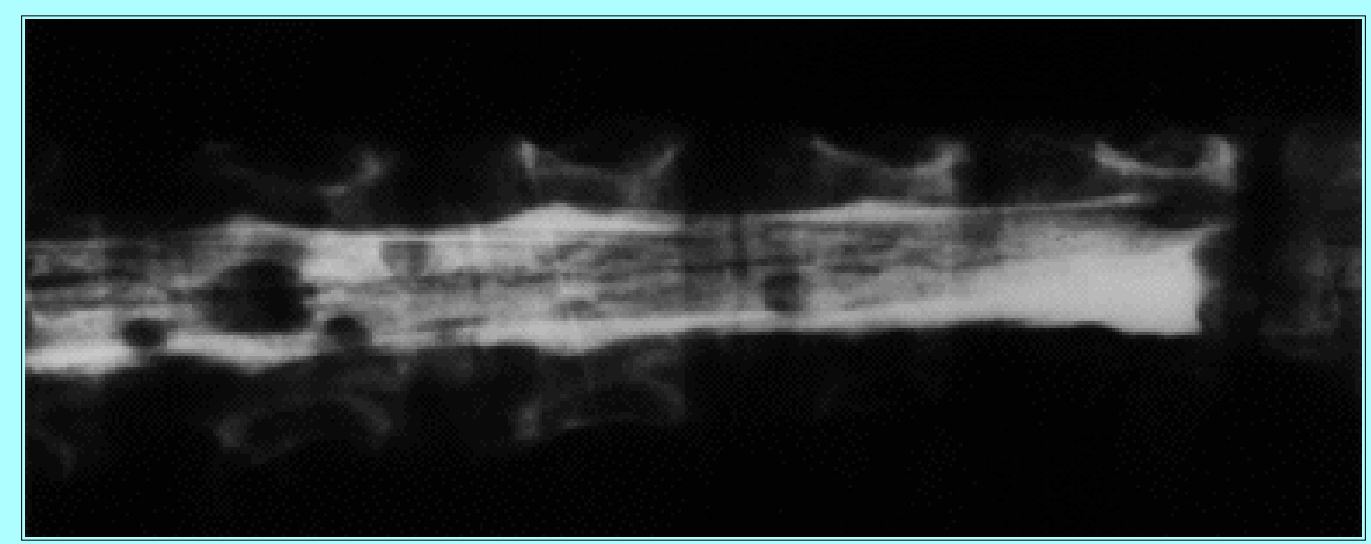

Fig. 14. Case 3. Myelogram demonstrating a complete block at T-8 and the spinal cord has shifted to the left. Note the additional smaller tumors at T-9 and T-11.

Other small lesions were seen at the levels of L-4, L-5, and S-1. A CT scan of the cervical spine, taken 5 hours after lumbar myelography with metrizamide, revealed no cervical metrizamide. Because of the possibility of cervical lesions, a cisternal myelogram was obtained. At the level of T-3 there were two tiny round densities of calcifications noted on the left side posteriorly, with no evidence of displacement or compression. A T6-8 and a T-11 laminectomy were then performed. Meningiomas were removed from T-6, T-7, and T-11, and neuromas from T-6 and T-12. The T-7 meningioma had caused the myelographic block (Fig. 15). Postoperatively, the patient did well and was able to move all extremities with $5 / 5$ strength, except for the previously discovered foot drop. The patient was discharged and readmitted 1 month later for removal of acoustic neuroma.

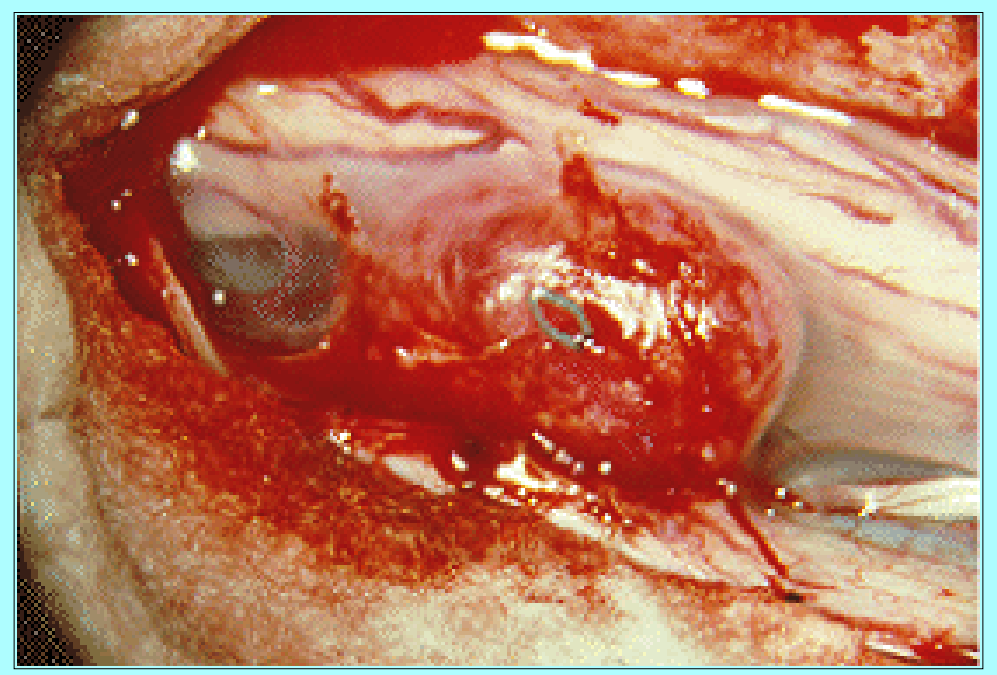

Fig. 15. Case 3. Intraoperative photograph showing a meningioma compressing the spinal cord from right posterior dural attachment. The photographic field is $35 \mathrm{~mm}$ in diameter.

Operation. On readmission there was a partial left facial and a complete left eighth. Motor examination was 5/5 throughout except for the complete left foot drop, and she was otherwise essentially normal neurologically. The patient underwent a right suboccipital craniectomy under the surgical microscope. The duration of the procedure was approximately 5 hours. A psammomatous meningioma, 5 to $6 \mathrm{~mm}$ in diameter and arising at the edge of the jugular foramen, was resected. The acoustic neuroma was completely resected and had measured $3 \mathrm{~cm}$ in the extracanalicular portion. The neuroma had extended into the auditory canal all the way to the crista, a distance of $12 \mathrm{~mm}$. The facial nerve was spared as was the auditory nerve, although the auditory fibers had been quite adherent and distorted, requiring a good 
deal of manipulation to free the fascicles. Additional small meningiomas were found forming a cluster of psammoma bodies approximately $1 \mathrm{~cm}$ in diameter and $4 \mathrm{~cm}$ in length, arising from the tentorium adjacent to the fourth cranial nerve. These tumors were also removed. The patient remained stable throughout the procedure.

Postoperatively, she was arousable, complaining of thirst and some incisional pain, but her neurological status was devastating. She had only upper extremity movement but no wrist or hand power, and both lower extremities were totally paralyzed. Her respirations were mainly diaphragmatic with just enough thoracic movement to prevent respiratory problem. Although her face moved well, she retained only minimal hearing in the right ear, with no speech discrimination. Emergency cisternal myelography was performed with 5-mm CT cuts that revealed an intraspinal meningioma at the levels of T-3, T-4 posteriorly on the left side, extending across the midline to the opposite side, as well as a defect ventrally at $\mathrm{C}-1$, but there was no block.

Second Operation. A C-1 and C-2 laminectomy and T-3 and T-4 laminectomy were performed. On the right side there was a neuroma of the posterior root of C-2 extending ventrally from the root and depressing the left dentate ligament all the way to the ventral dura. The tumor was opened and partly cored, sufficient to allow its removal, and was then resected with one fascicle. The dura was then opened at the T-3 level. There was a meningioma dorsally and a neuroma arising from the T-3 nerve root, and the two tumors together had formed a fairly deep cavity in the dorsolateral aspect of the cord at T-3. At the T-4 level there was another meningioma approximately $5 \mathrm{~mm}$ in diameter that had not been indenting the cord at all. The patient tolerated the procedure and was returned to the ICU in stable condition, still virtually unchanged from her preoperative status.

Postoperative Course. Throughout the remaining hospital stay there was only minimal progress. Wrist movement returned to $3 / 5$, biceps to $4 / 5$, but there was no fine finger movement, and the lower extremities remained flaccid with minimal ankle movement. There was a C-7 sensory level of fairly severe degree, and only minimal proprioception at the ankles, mainly on the right.

She was remarkable for her level of active participation in her rehabilitation as well as the degree of cooperation she received from her parents and pediatrician, and she made sufficient progress so that she was able to go to school in her wheelchair, supplementing her minimal hearing by lip reading; she was getting along reasonably well. The final episode in this tragedy was that 4 years later she was unexplainably found dead in her college room. I do not believe that any case in all my years of practice has depressed me to the degree that the death of this very special young woman did, and scarcely a day goes by that I still do not think of her.

Since the time of this case, every patient, regardless of diagnosis, who is to be operated on in neck flexion or extension has somatosensory evoked potentials from tibial nerve stimulation recorded at the scalp and the latency measured. They are then placed in the desired position for operation and the evoked potentials repeated to confirm that the latency has not increased. If there is a change, the position is altered until the latency returns to baseline.

\section{CASE 4}

History. This Caucasian, right-handed woman was 21 years old when she first came under my care. Seven years previously, atrophy of the right masseter muscle was noted. Two year later a tumor of the right ulnar nerve was removed and diagnosed as neurofibroma, although it was probably schwannoma. 
She developed diplopia on left lateral gaze, and a tumor of the left sixth cranial nerve was suspected; a diagnosis of von Recklinghausen's disease was made. She developed hearing loss and tinnitus on the right side. A myeloencephalogram showed bilateral acoustic neuromas and raised suspicion of pathology in the upper cervical spine.

Presentation and Neuroimaging Studies. She was then referred and admitted to MSH and subsequently had five admissions. On her first admission, she presented with deafness in the right ear and markedly reduced hearing in the left. There was a left sixth cranial nerve palsy, early seventh, and superior division of left third cranial nerve weakness. Angiography, myelography, and CT scanning showed meningioma, left C1-2 bilateral acoustic neuromas, right larger than left, left temporal meningioma, and a small left lateral ventricle meningioma. Despite all this she was a cheerful, pleasant, and charming young woman.

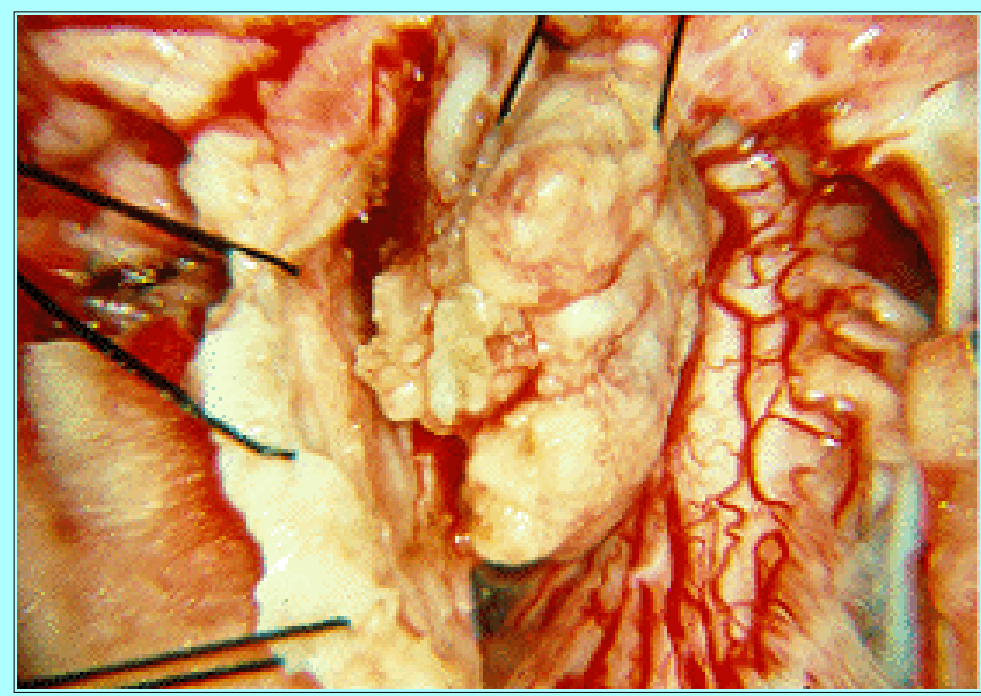

Fig. 16. Case 4. Intraoperative photograph showing a left-sided meningioma being removed at $\mathrm{C} 1-2$. The photographic field is $35 \mathrm{~mm}$ in diameter.

First and Second Operations. A cervical laminectomy with removal of meningioma at C1-2 on the left (Fig. 16) and neuroma removal at C2-3 on the left and C-2 on the right was performed. Readmitted a few months later, she underwent removal of her right acoustic neuroma, and small fifth, seventh, and ninth cranial nerve neuromas (Fig. 17 left and right). Admitted again some months later, she underwent removal of left acoustic neuroma and removal of tentorial meningioma. Postoperatively, the patient had complete hearing loss on the left superimposed on the hearing loss on the right. She also had partial left facial weakness.

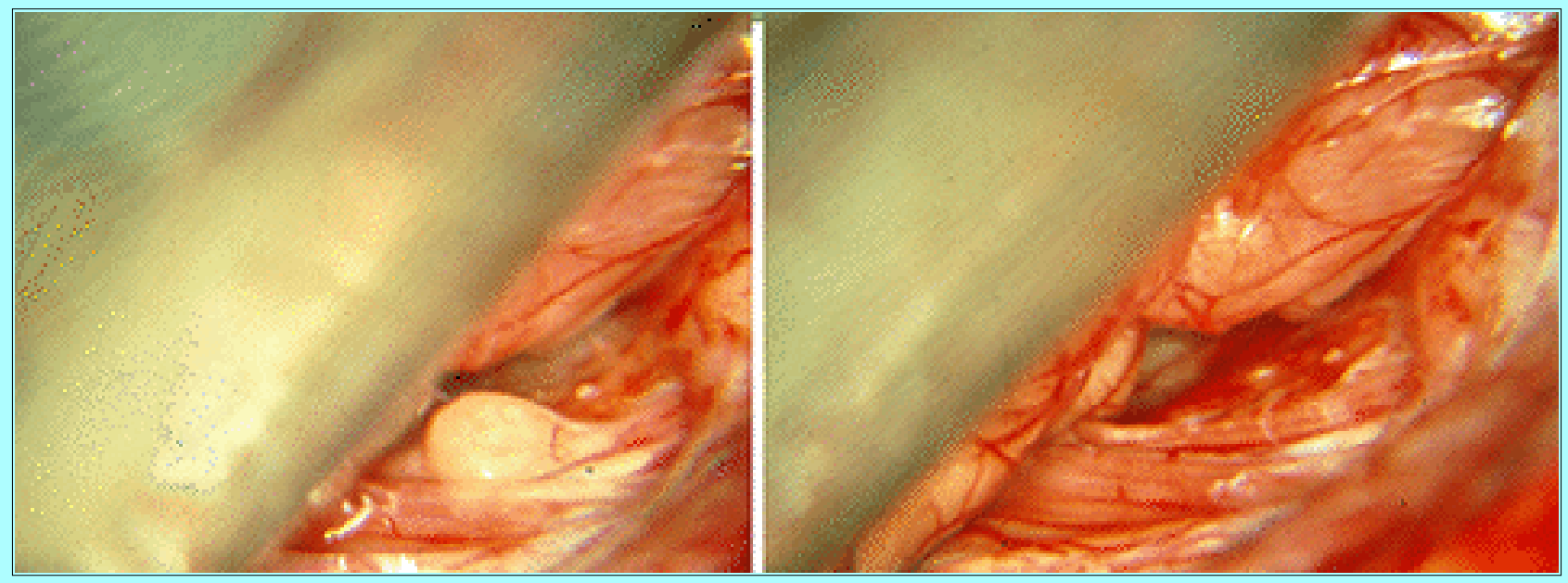


Fig. 17. Case 4. Intraoperative photographs showing a 4-mm schwannoma among the ninth and 10th cranial nerves (left) and the same view after removal (right). The large acoustic neuroma is seen in the upper right corner. The photographic field is $24 \mathrm{~mm}$ in diameter.

Examination and Postoperative Course. She was admitted for evaluation 1 year later. There were no new complaints. The scars of previous bilateral suboccipital craniectomies, sunken wounds, were well healed. The neck showed scarring of previous cervical laminectomy. The rest of the examination was normal except for small café-au-lait spots on posterior aspect of left arm and right buttock and the scar of previous surgery on the right hand. Gait was normal, even on tandem. On Romberg testing, there was minimal oscillation without falling. She was lucid, coherent and well oriented, with no memory deficit. Speech was normal. Cranial nerves showed visual acuity 20/20 OD, 20/30 OS, with no visual field defect or papilledema. The left pupil was nonreactive to light or accommodation. The left eye could move medially and downward but with limited movement. The fourth cranial nerve on the left was involved, with no rotation of eye globe. There was no nystagmus. First division of the fifth cranial nerve on left had decreased pinprick and touch, and corneal reflex was decreased on the left. Atrophy of masseter muscle on the right and deviation of jaw to the right on opening the mouth was noted. There was partial facial weakness on the left and complete hearing loss bilaterally. The soft palate was symmetrical with depressed gag reflex bilaterally. She could barely feel touch in soft palate. The tongue was symmetrical. Power was $5 / 5$ in all extremities. Deep tendon reflexes were symmetrical and two plus throughout with no Babinski or Hoffman. Coordination testing showed no deficit. Sensory was intact to pinprick, touch, and position. A CT scan demonstrated postoperative changes of bilateral suboccipital craniectomy in addition to a small left lateral ventricle tumor, probably a meningioma, and a tentorial notch meningioma seen in the last surgery on the right side. Because there was some doubt concerning the possibility that there might be increase in size of the lesions, a cerebral angiogram was obtained which showed that the tumors seen on CT scan were not significantly larger than previously seen in studies performed at the time of last surgery.

Ten years later there had been no progression of her tumors, she was teaching in a school for the deaf, and she was married. Despite all of the information and counseling she had been given, she was overjoyed to announce that she was now pregnant.

\section{CASE 5}

History and Presentation. When first seen 20 years ago this patient, a 13-year-old, right-handed Caucasian girl, was diagnosed as having bilateral acoustic neuromas when she began to develop a wobbling gait and difficulty hearing in the right ear. She developed difficulty swallowing and speaking, then occipital headaches and vomiting. Admitted to $\mathrm{MSH}$, she was found to have a left sixth cranial nerve palsy, papilledema, and bilateral lateral gaze nystagmus. Also noted was left facial weakness and poor tandem gait with positive Romberg sign. There was dysmetria and diffuse hyperreflexia.

Neuroimaging Findings. A CT scan and angiogram confirmed bilateral acoustic neuromas, $2.5 \mathrm{~cm}$ on the left and $4 \mathrm{~cm}$ on the right, along with moderate hydrocephalus (Fig. 18 left). Also noted was a small left tentorial meningioma. The CT scan of the spine was essentially normal and the angiogram showed that the right SCA was pushed medially and upward and the right AICA was displaced posteriorly. The left AICA was posterior and inferior to the left tumor. Audiometry revealed no hearing on the right and 60-db loss on the left. Her behavior was extremely strange: at no time would she speak to or answer any of the hospital staff, although she was said to speak to her parents when no one else was present, and she 
had been going to school.
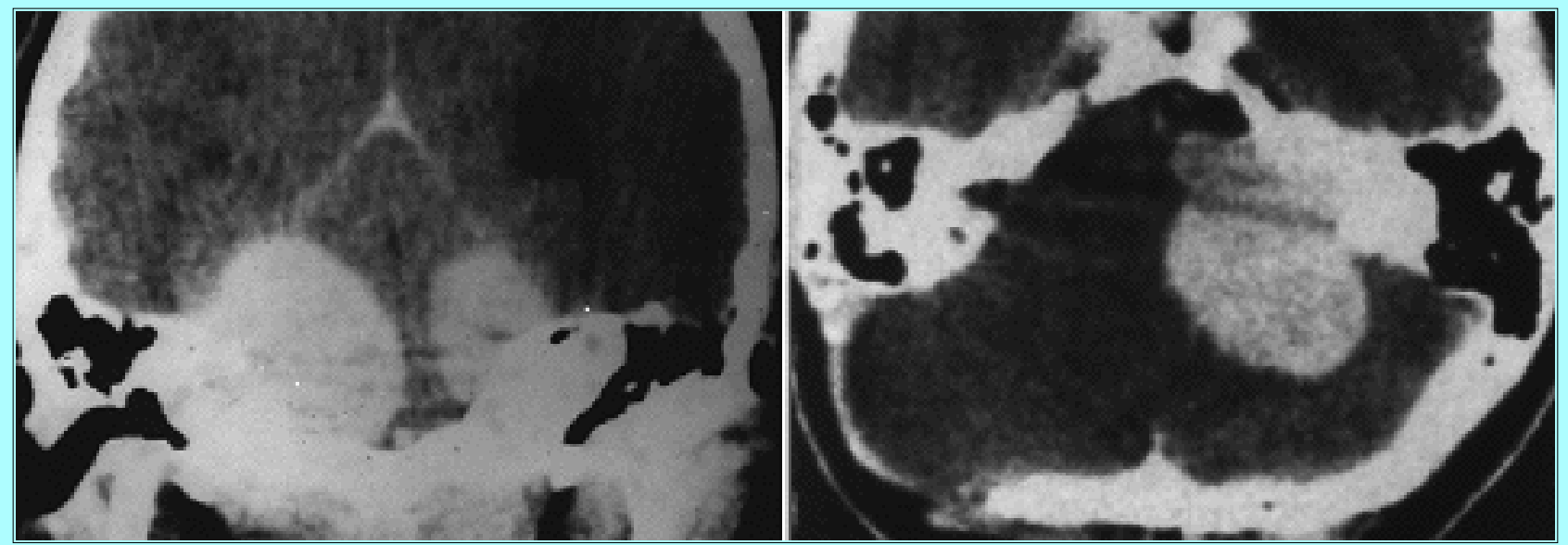

Fig. 18. Case 5. Computerized tomography scans demonstrating a 4-cm right-side acoustic neuroma and a $2.5 \mathrm{~cm}$ left-sided acoustic neuroma (left). After removal of the right-side tumor (right). Note that the left-sided neuroma has enlarged to $4 \mathrm{~cm}$.

Operation. A right suboccipital craniectomy and removal of the right acoustic neuroma was carried out. The tumor was a typical acoustic neuroma with fibrous capsule and a relatively vascular interior displacing the fifth cranial nerve upward, the seventh and eighth cranial nerves forward, and the ninth, 10th and 11th cranial nerves downward. The tumor extended from the foramen magnum to the tentorial notch. The auditory and both vestibular nerves along with the facial nerve were grossly involved with tumor. A segment of the facial nerve was resected and a primary end-to-end anastomosis carried out.

Postoperatively, the patient did well. Tarsorrhaphy was necessary on the right side for the seventh cranial nerve palsy. After a short period of time, balance difficulty and speech improved greatly. She had no double vision, headaches, or swallowing difficulty and was able to jump rope without difficulty. The patient returned to her eighth grade class and achieved honors despite her hearing deficit. Because the likelihood of hearing preservation seemed so poor with a $60-\mathrm{db}$ loss in the remaining tumor-bearing left ear, further surgery was delayed.

Second Operation. A follow-up CT scan showed a marked increase in the size of the left acoustic neuroma to $4 \mathrm{~cm}$ only 8 months later (Fig. 18 right), and she was readmitted for surgery. At that time there was no hearing on the right and more than 60-db loss in the left ear. Visual acuity was 20/50 bilaterally. There was decreased right corneal reflex. Right facial palsy was beginning to recover, and there was a left facial weakness. Her gag reflex was good. There was no coordination or gait difficulty. Mental status was normal. A left suboccipital craniectomy and removal of acoustic neuroma were carried out. The tumor was a 4-cm fibrous, vascular, yellow-tan typical acoustic neuroma arising from the superior vestibular nerve, displacing the fifth cranial nerve anteriorly, the seventh and eighth cranial nerves anteriorly and inferiorly, and the ninth, 10th, and 11th cranial nerves downward. A small tentorial notch meningioma was also removed from above the fifth cranial nerve, and this portion of the tentorium was resected as well (Fig. 19). All cranial nerves except the superior vestibular were preserved and the patient did quite well. 


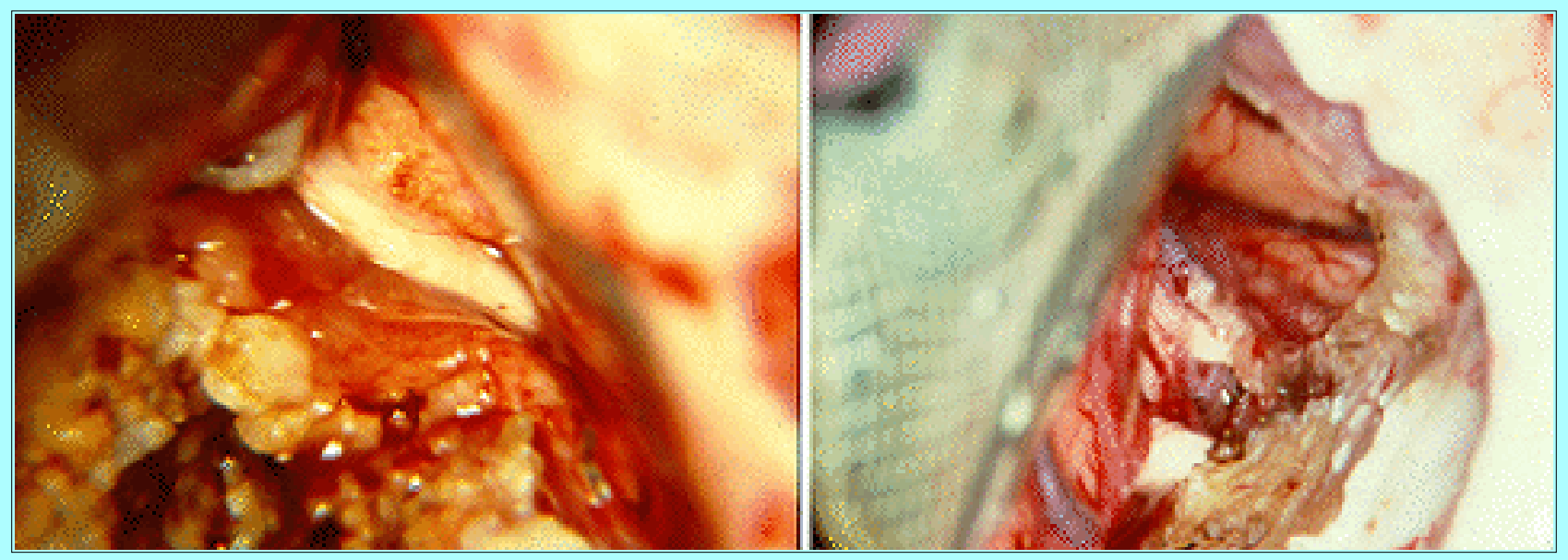

Fig. 19. Case 5. Left: Intraoperative phototraph showin an acoustic neuroma just below the fifth cranial nerve and a small tan meningioma between the fifth nerve and the tentorial edge. Right: Intraoperative photograph showing that the acoustic neuroma, meningioma, and a portion of the tentorium have been resected. The photographic fields are is $25 \mathrm{~mm}$ and 36 $\mathrm{mm}$, respectively.

Postoperative Course. On postoperative examination the patient did have minimal hearing in the left ear, and there was no left facial palsy. It should be noted that the patient's personality made it very difficult to adequately evaluate her auditory acuity or to determine how functional it would be. Postoperative scanning showed no tumors (Fig. 20), and over the ensuing years no other tumors appeared. She made an excellent recovery from the end-to-end facial nerve anastomosis, and her facial movements are now symmetrical. She has adapted very successfully and went to college for the deaf, because her remaining hearing was not really adequate, although it aids in her lip reading. She has become expert at signing and writes charming letters to me, having overcome her unwillingness to communicate.

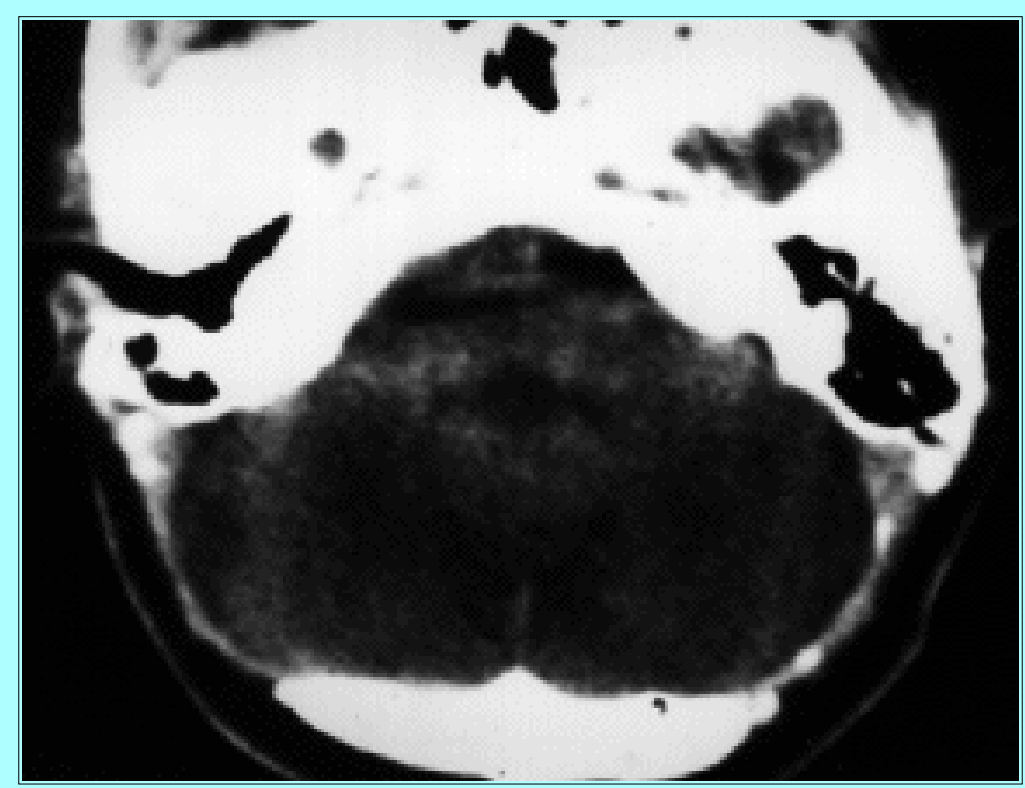

Fig. 20. Case 5. A CT scan obtained after surgery, demonstrating no additional tumors.

\section{CASE 6}

History and Presentation. This 16-year-old Caucasian boy had an ear infection on the left side. After failure of the drum to heal, a biopsy of the area was performed and reported as a desmoplastic reaction. 
He then developed visual blurring and severe papilledema was discovered. A CT scan was reported to show a large left acoustic neuroma, and the patient was referred to MSH. On admission, he presented with severe papilledema with hemorrhages. He was deaf in the left ear and had atrophy of the left side of the tongue, but he was otherwise quite normal neurologically. Hearing in the right ear was normal. He had been going to school until just prior to admission. There were no cutaneous lesions. The rather primitive CT scanning machine then in use showed bilateral acoustic neuromas, $5 \mathrm{~cm}$ on the left and 2 $\mathrm{cm}$ on the right. There was also dural thickening in the posterior fossa that was ill defined, perhaps due to the limitation of the machine. Review of the middle ear biopsy that had been diagnosed as a desmoplastic reaction revealed that it was actually a meningioma.

Operation. With the diagnosis of central NF a left suboccipital craniectomy and removal of the left acoustic neuroma were planned. After medical clearance and all of the standard preparation, in the usual semisitting position, a vertical skin incision $12 \mathrm{~cm}$ long was made $2 \mathrm{~cm}$ medial to the left mastoid. The incision was carried down through the galea, deep fascia, and muscles, and the suboccipital bone and mastoid were visualized by subperiosteal dissection. A burr hole was made and enlarged to form a 4.5-cm suboccipital craniectomy including the entire mastoid process. The sigmoid sinus was completely exposed and noted to be bulging, even though the G-suit pressure was completely down. The mastoid cells were sealed with bone wax. The sinus was then covered with gelfoam.

A semicircular dural opening with its base medially was made as were lateral relaxing incisions: one up to the transverse and one down to the sigmoid sinus. Traction sutures were used to sew the lateral dural segment to the muscles and then used to draw the lateral sinus out of the wound over the cut surface of the mastoid. The loupes and headlamps were removed, and the remainder of the operative procedure was carried out under the Zeiss operating microscope at magnifications of 10, 16, and 25 diameters.

The Malis self-retracting brain retractor was now placed and used to elevate the cerebellum in the plane of the petrous pyramid. This immediately demonstrated the presence of fungating meningioma, unencapsulated, extending from down in the jugular foramen medially toward the foramen magnum. This layer of meningioma was several millimeters thick and approximately $2 \mathrm{~cm}$ in length, extending forward where it ran underneath the posterior surface of the large acoustic neuroma. At this time, the nerves of the jugular foramen were partly concealed. Using the bipolar coagulator, a plane of dissection along the dura was carried out from near the foramen magnum upward toward the jugular foramen. The 11 th cranial nerve had been displaced medially and anteriorly and was exposed in the course of the resection of this carpetlike meningioma. The 11th cranial nerve was grossly abnormal, five or more times its ordinary diameter, and nodular with infiltration by neuromas of all of its fibers. Going upward from the jugular foramen, multiple small tumor nodules were seen in the ninth and 10th cranial nerves and the meningioma surrounded these nerves.

With prolonged microdissection, the meningiomatous tissue was resected in order to more adequately visualize the neural structures. Because of the attachment of the acoustic neuroma from above, which obscured the visualization, a strip of glove rubber was placed temporarily and attention was given to the acoustic tumor. Here, the arachnoid layer could be separated and followed upward to the tentorium where it was now possible to free the posterior capsule from the arachnoid to the extent to which approximately $1.5 \mathrm{~cm}$ of space was achieved. The tumor capsule was incised using the bipolar coagulator. The content of the tumor was typical yellowish acoustic neuroma material of moderate vascularity. Coring of the tumor was carried out with the bipolar coagulator in the usual manner, necrosing small portions of the tumor with the bipolar coagulator at low voltage under continuous saline 
irrigation and then removing these sections either with suction or sharp dissection.

This coring provided enough additional room to be able to elevate the inferior surface of the acoustic tumor sufficiently to permit separation medially along the ninth cranial nerve back to the brainstem. Here, the acoustic tumor extended over the foramen magnum, and coring of this area was complicated by the presence of large arterialized veins, which were progressively sealed with the bipolar coagulator and cut. Now attention could be given to the ninth, 10th and 11th cranial nerves and it could now be seen that there were indeed four separate meningiomas that had been in this area. Two of the posterior petrous pyramid tumors posterior to the jugular foramen had been resected; a third meningioma extended under the ninth, 10th and 11th cranial nerves and down into the foramen rim; a fourth meningioma, separate from the one between the ninth, 10th and 11th cranial nerves with only a few millimeters between, however, surrounded these nerves, entering and filling the jugular foramen. This tumor, which was soft, friable, and totally unencapsulated, was resected bit by bit from between the neural fascicles down into the jugular foramen.

The ninth cranial nerve had only one significant neuroma in it, a nodule about 4 or $5 \mathrm{~mm}$ in diameter in a single fascicle. This was resected with its fascicle. There were a number of $0.5-\mathrm{mm}$ or so enlargements of other fascicles, and these were left intact. There were also minute involvements of fascicles of the 10th cranial nerve but no tumor larger than a millimeter, and these were left undisturbed. The 11th cranial nerve, as already stated, had been entirely replaced by a fusiform neuroma arising from the spinal area in the foramen magnum, but there were a few fascicles coming from the brainstem that were intact. The spinal portion was resected completely with its tumor. This area was now temporarily covered with a sheet of glove rubber placed to also cover the PICA, which had been dissected free medially as it came off the vertebral artery near the 12th cranial nerve. There was no intracranial tumor of the 12th cranial nerve, despite the fact that it was known to be affected with complete atrophy of the left side of the tongue, the involvement of which was now assumed to be entirely extracranial.

Superiorly, the petrosal vein and vein of the lateral recess had been pushed forward by the tumor; they were encountered coming backward and downward along the tentorium at the junction with the petrous pyramid as this portion of the capsule was followed upward along the tentorium. Because of the distortion and stretch of these veins, it was not necessary to occlude them. As coring of the tumor continued, the medial capsule was separated from the brainstem with marked adherence of arachnoid capsule and pia together, requiring separation of brainstem pia in areas to permit complete removal. Both vestibular nerves were totally unidentifiable as the tumor had engulfed them right into the brainstem pia. The tumor was lobulated as though it had multicentric origins that had fused together, with neural and vascular structures engulfed in the layers.

As the dissection continued forward the facial nerve could now be seen as a tan trunk extending forward along the brainstem and the auditory fibers could be seen curving downward toward the ninth cranial nerve. As the tumor capsule was brought down from the tentorium, the division was carried forward and the capsule was separated from the fourth cranial nerve, the SCA, and the free margin of the tentorium; then the curved, distorted fifth cranial nerve was brought into view with the division of the rest of the superior surface of the capsule, which extended up above the fifth cranial nerve along the peduncle. The origin of the fifth cranial nerve from the brainstem was now separated from the tumor, and the separation of the fifth cranial nerve was carried around to its prior visualization where the capsule had been resected superiorly. The nerve had been not only pushed well forward but had been pushed somewhat downward and the tumor had gone up over the fifth cranial nerve along the upper brainstem for approximately an 
additional 1.5 to $2 \mathrm{~cm}$. The seventh cranial nerve had an abrupt angle downward and laterally and curved along the inferior portion of the tumor. This large tumor had pressed the brainstem across the midline to the opposite side, exposing the BA. The tumor capsule was now separated from the BA progressively upward and the sixth cranial nerve was visualized, having been drawn back across the BA and crossing the BA back toward the point of exit through the dura to the cavernous sinus. The sixth cranial nerve crossed the BA with a characteristic bend that was stretched over the origin of the AICA. The AICA looped laterally toward the jugular foramen, then back to the brainstem in front of the flocculus. The seventh cranial nerve crossed the BA, then the sixth cranial nerve, and then turned backward and upward laterally toward the IAM. This area was freed by further resection of the tumor, carrying the resection up along the IAM and along the area at which the fifth cranial nerve, curving backward from where it had been displaced, now had to make an abrupt angle forward, entering Meckel's cave.

This completed the removal of the tumor medially, leaving the small lateral segment protruding from the IAM. The tumor measured $5 \mathrm{~cm}$ in diameter. The brainstem, despite the dissection, appeared to be normal in its vascularity and, although quite deeply cupped, untraumatized.

A triangular-shaped segment of dura, its base at the IAM and its apex at the sigmoid, was now coagulated and resected. Using the high-speed diamond burr drill, the posterior wall of the IAC was drilled out to reach the region of the crista laterally, a distance of approximately $14 \mathrm{~mm}$. Vertically, the drilling was carried to the height of the tumor expansion, approximately $10 \mathrm{~mm}$. The dura was opened within the drilled-out canal, and the canal was found to be filled with tumor. Dissection around the tumor permitted visualization of the inferior vestibular, auditory, and facial nerves anterior and inferior to the tumor. The tumor was reflected laterally, separating it from the seventh cranial, the inferior vestibular, and the auditory nerves. The superior vestibular nerve, from which the tumor originated, was sectioned at the crista; the inferior vestibular nerve, which was totally engulfed by tumor, was also sectioned at the crista. The facial nerve was freed as it ran along the upper anterior wall of the IAC. Although the facial nerve had been densely adherent and separation of tumor from within its fascicles had caused considerable disruption, it was in anatomical continuity. The auditory nerve fibers had been badly splayed and anatomically disrupted to the extent to which function could not be expected.

The drilled-out area in the IAC was now filled with a fat pad from the subcutaneous layer, which was sutured into place with No. 6-0 monofilament to the dura. Prior to the closure, inspection demonstrated that complete homeostasis had been achieved. There were, however, two bulges up in the tentorial surface that had been exposed: one beginning just above the IAM (approximately $2 \mathrm{~cm}$ in diameter and approximately $0.5 \mathrm{~cm}$ thick); the other separated from this and anterior to it by about $1 \mathrm{~cm}$ which went right up to the tentorial edge (this was approximately the same size as the first one and appeared to be within the tentorial leaves). The inferior layer of the dura was incised over the lower one, and a whitish tumor was found between the upper and lower surface of the tentorium, thickening the tentorium to more than $1 \mathrm{~cm}$. This material was separated from within the tentorial surfaces using the bipolar coagulator to free it, separate it, and produce the hemostasis. It appeared to be neuromatous in its gross anatomical character yet, strangely, was completely enclosed within the dural leaves of the tentorium. Exactly the same appearance was noted on the more anterior lesion that rounded the tentorial edge into a more than $1-\mathrm{cm}$ thickness. This, too, seemed to be an intratentorial leaf neuroma. Resection of both of these apparent neuromas was complete. Neuropathological examination confirmed that these were schwannomas. The self-retaining brain retractor was removed and the cerebellum allowed to return to its normal position. The cerebellum appeared to be untraumatized. Dural closure was now carried out using No. 4-0 Nurolon. The dural surface was covered with a sheet of gelfoam. A Hemovac drain was brought 
in through a separate stab wound to the epidural surface. The usual layer-closure procedure of the wound was carried out using No. 2-0 interrupted Nurolon in multiple layers for the muscles and deep fascia and No. 3-0 interrupted Nurolon in separate layers for the galea, the subcuticular layer, and the skin. Blood loss was estimated at $500 \mathrm{ml}$ and was not replaced. The patient tolerated this 13-hour microsurgical procedure well and was sent to the ICU in good condition, reacted well, and was responsive.

Postoperatively, his immediate course was uncomplicated. His facial function had been preserved, his papilledema was subsiding, and he returned to school. Surgery for the right acoustic neuroma was refused, on the basis that this was his only remaining hearing ear.

Postoperative Course. Six months later, he experienced thoracic back pain accompanied by leg weakness. A completely blocking intradural tumor was found on study. Laminectomy revealed a meningioma extending over T-5 and T-6 which was completely removed, relieving the symptoms. Only 3 months later papilledema recurred, and an angiogram indicated bilateral jugular foramen obstruction, so a lumboperitoneal shunt was performed. After very brief initial relief, his course rapidly deteriorated. Bilateral facial palsy developed, along with inability to swallow, thus requiring a gastric tube. He then developed uncontrollable grand mal seizures, and died in a seizure 1 year after the first surgery.

Autopsy Findings. At autopsy the entire calvarial dura was found to be covered with innumerable meningiomas, both large and small (Fig. 21 left). Both sigmoid sinuses, both lateral sinuses, and the sagittal sinus were invaded by meningiomas. Meningiomas from the jugular foramen had grown down into the retropharyngeal space bilaterally. There were also many neuromas, although the left acoustic neuroma had not recurred, and the unoperated right acoustic neuroma had reached $3 \mathrm{~cm}$. His spinal nerves including the entire cauda equina were studded with schwannomas (Fig. 21 right). There was a meningioma in the lower lobe of the right lung and a schwannoma in the lower lobe of the left lung.
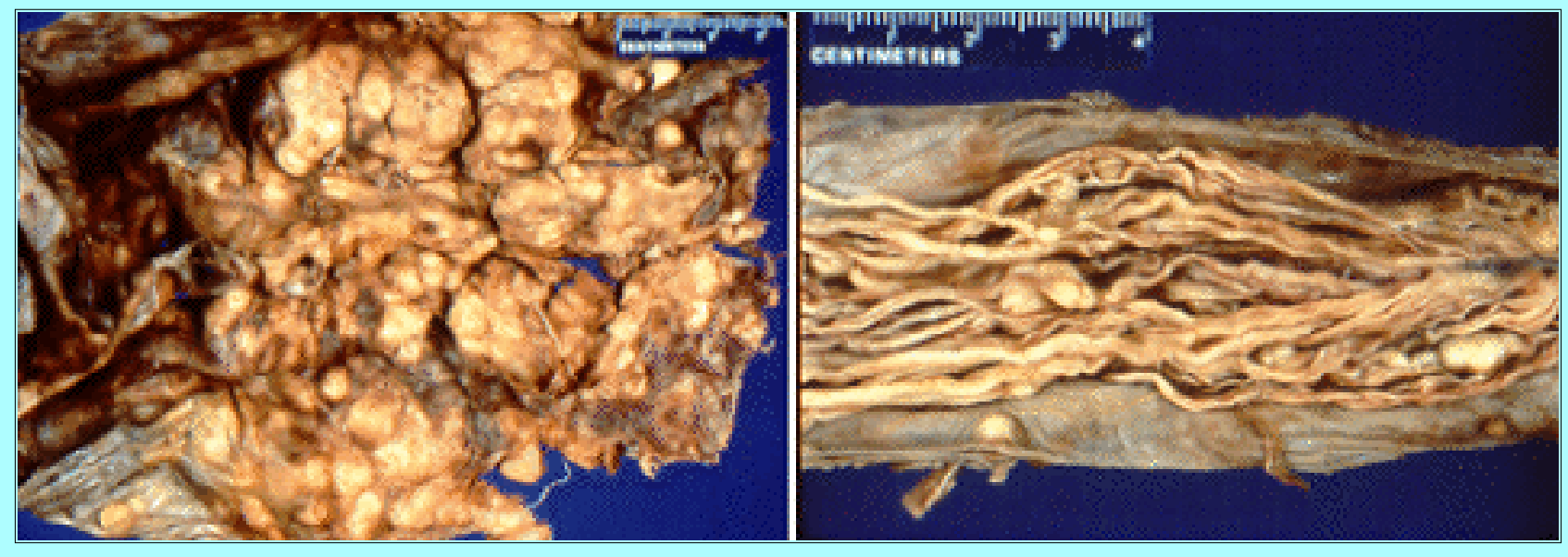

Fig. 21. Case 6. Photographs obtained at autopsy showing the inner aspect of the calvarial dura covered entirely with meningiomas (left) and multiple schwannomas involving the cauda equina (right).

\section{CASE 7}

History. This 32-year-old Caucasian man had noted right hearing loss and then right shoulder weakness. Bilateral acoustic neuromas and a tumor at C1-2 were diagnosed. Partial removal of the right acoustic neuroma was carried out in another country, and later followed by removal of a small C-2 meningioma. At that operation widening of the cord was noted, and the patient was referred for further care.

Presentation and Neuroimaging Findings. He was then admitted to MSH. On admission he was deaf in 
the right ear, with excellent hearing in the left ear. There was mild right facial weakness. The left arm was weak in all muscle groups, proximal more than distal. There was moderate weakness in the left leg, although gait was normal. There was a cape sensory decrease from the upper cervical area down to T-8, with essentially normal sensation below. Imaging studies demonstrated a very small left acoustic neuroma and a moderate residual right acoustic neuroma. Contrast-enhanced MR imaging showed marked enlargement of the cervical spinal cord from the foramen magnum down, with unusual zones of enhancement (Fig. 22). Because the intramedullary tumor was considered to pose a major problem, cervicothoracic laminectomy was scheduled.

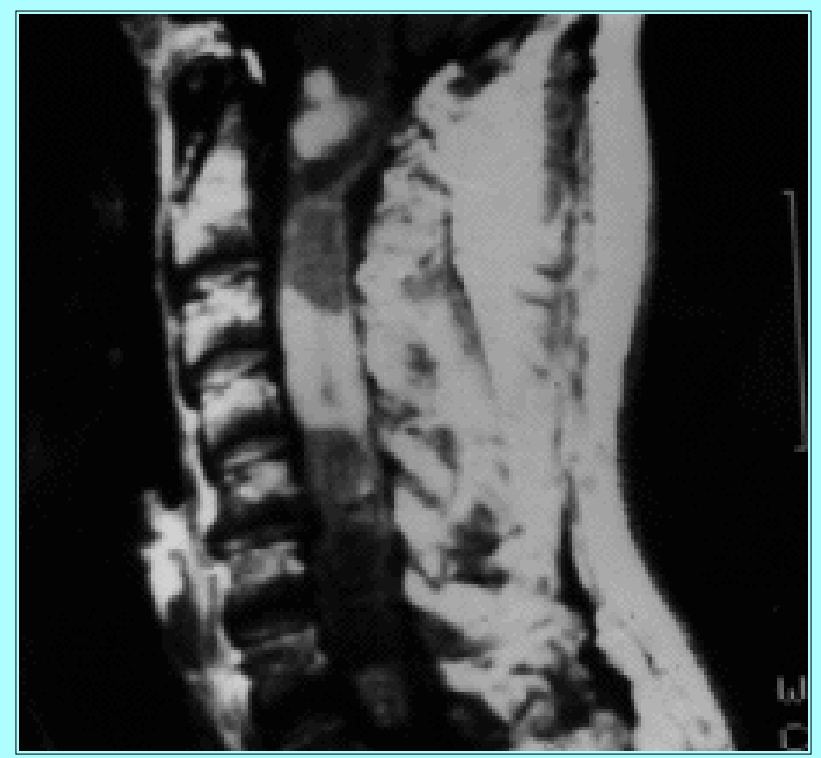

Fig. 22. Case 7. A gadolinium-enhanced MR image of the cervical spine showing irregular nodular enhancement of the enlarged cord.

Operation. The surgical procedure was carried out in the 45 š prone oblique position with the left side up and the patient's head supported in the Gardner pinned head rest, as well as all the standard preoperative procedures used in the rest of this series The well-healed upper cervical incision was reincised and extended to include a new midline incision from the inion to the level of T-3. The heavy scar from C1-4 was reincised, whereas from $\mathrm{C}-4$ downward subperiosteal dissection exposed the processes and laminae. The previous partial laminectomies of $\mathrm{C}-1, \mathrm{C}-2$, and $\mathrm{C}-3$ were now widened and the medial aspect of the previous right suboccipital craniectomy demonstrated. Eventually, the entire area down through and including T-1 up through the foramen magnum rim was widely exposed bilaterally using en face rongeuring. After hemostasis was achieved and a suction sump placed, the dura was opened from C1-7 in the midline. On the left side at C-2, there was a silastic dural patch that was markedly bowed inward, trapping a layer of fluid between it and a neodura which had developed external to it. The sutures holding the silastic patch were cut and it was removed. The dural margins were now sewn over the cut bony edges of the laminectomy to the adjacent musculature for hemostasis.

Up to this point, the operation had been carried out with magnifying loupes and the fiberoptic headlamps. The Zeiss operating microscope was now brought into the field, and the remainder of the procedure was carried out under the microscope at magnifications of 10,16, and 25 diameters. The spinal cord was markedly but irregulary widened with an abnormally heavy vascular pattern posteriorly throughout the exposure (Fig. 23 left); it tapered at the upper end, at the foramen magnum, and at the lower end below C-7. On the left side at C-2, the posterior root was missing, apparently from the prior laminectomy and removal of the meningioma. Here the cord was adherent to the arachnoid and to the dura. The widened 
cord filled the canal all the way down, but the dorsal columns were not widened, and the cord was stretched out below the dorsal root entry zone. The greatest widening was at the C-5 level. A midline myelotomy was then performed and revealed the surface of an obvious tumor, brownish in color, without a capsule but with a plane of cleavage and with some cystic spaces in relation to it.
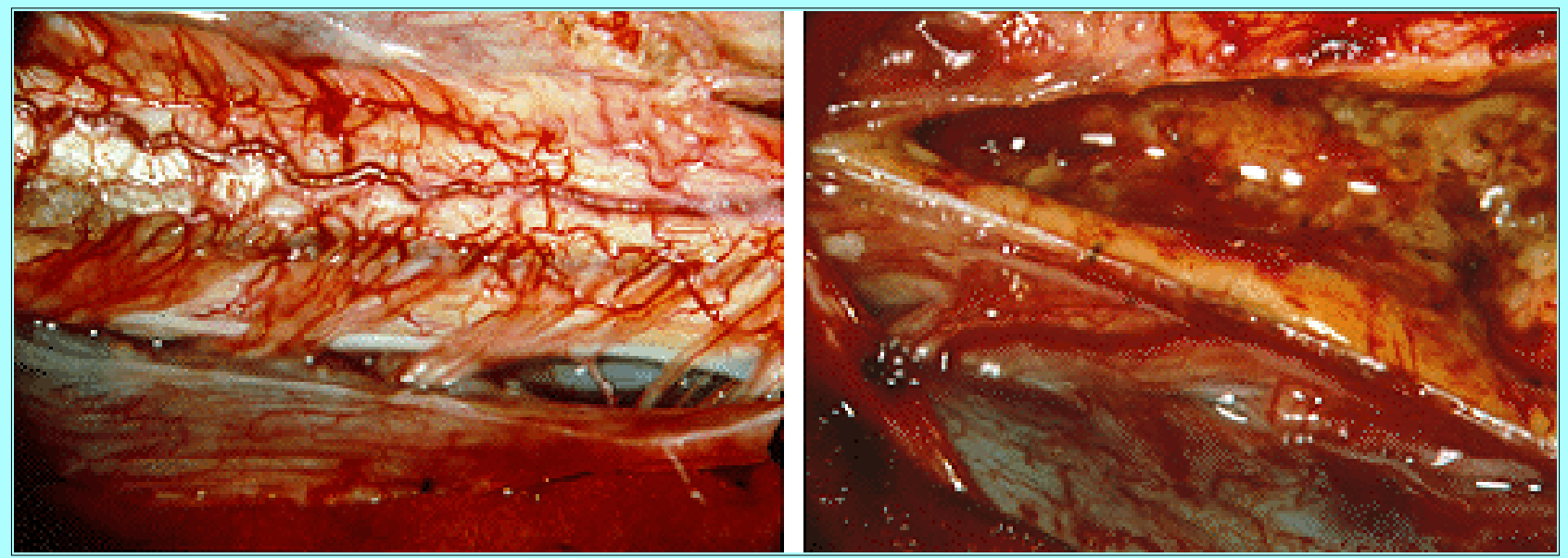

Fig. 23. Case 7. Intraoperative photographs showing an irregularly widened cord at C-5 (left) and a myelotomy held open with pial stay sutures while an unencapsulated ependymoma is removed (right). The photographic field is $36 \mathrm{~mm}$ in diameter.

Pial sutures of No. 6-0 monofilament to the dura were now used to suture the myelotomy open and the tumor at the C-5 level was cored and dissected using the bipolar coagulator (Fig. 23 right) and the intramedullary dissectors to free it in the hope that the widened areas above and below it were cystic only. After complete removal of this tumor, it was clear that this was not the case: just above and below it, the margins of what appeared to be separate tumors were noted.

As the myelotomy was extended upward and downward, multiple additional tumor nodules were found for a total seven separate lesions, some of which appeared almost in continuity and some of which were clearly separated, extending all the way up into the foramen magnum. As these tumor nodules were removed, it became clear that they differed considerably in their appearance and vascularity and separability: some appearing to be typical ependymoma, some to be astrocytic. Still others could well have been central neurinomas. Each was removed separately and sent as a separate specimen to the neuropathology laboratory. All were there confirmed as ependymomas. At the C-1 level, the intramedullary tumor was densely fibrous, approximately $1.5 \mathrm{~cm}$ in diameter and $2 \mathrm{~cm}$ in length, and arose from the ventral median raphe, approximately at the point at which the anterior spinal artery is formed by the descending branches of the two vertebral arteries. This ventral median raphe had been demonstrated with the removal of the last portion of the tumor with the cavity extending through the cord. This portion was most suggestive of a meningioma extending intramedullary from the midline anterior raphe but also proved to be an ependymoma. Total removal was achieved of all of these tumor masses, with the single myelotomy eventually running from its upper end just at the inferior margin of the fourth ventricle to the lower end of the C-7 vertebra, where the cord finally tapered to a normal appearance Two small dorsal root neuromas, only a few millimeters in diameter, were shelled out of the $\mathrm{C}-3$ root on the right, preserving the rest of the root.

The pial stay sutures were removed and the cord dropped together, somewhat narrower in diameter than normal, in contrast to its previous marked widening (Fig. 24). The dural stay sutures were cut and the dura closed with running sutures of No. 4-0 Nurolon. The dura was now covered with a sheet of gelfoam 
and the muscles and fascia closed, as well as the deep scar with interrupted sutures of No. 2-0 and No. 3-0 Nurolon after a Hemovac drain was brought in through a separate stab wound to the epidural space. The subcuticular layer was closed with inverted No. 3-0 Vycril sutures, and the skin was closed with No. 4-0 Nurolon. The patient tolerated this 14-hour microsurgical procedure well. Because of the possibility of sleep apnea, his endotracheal tube was left in place, although he was awakening well and moving both legs and his left arm. He removed the endotracheal tube himself on arrival at the ICU, and it was not replaced. Blood loss was estimated at 1 liter and was replaced.

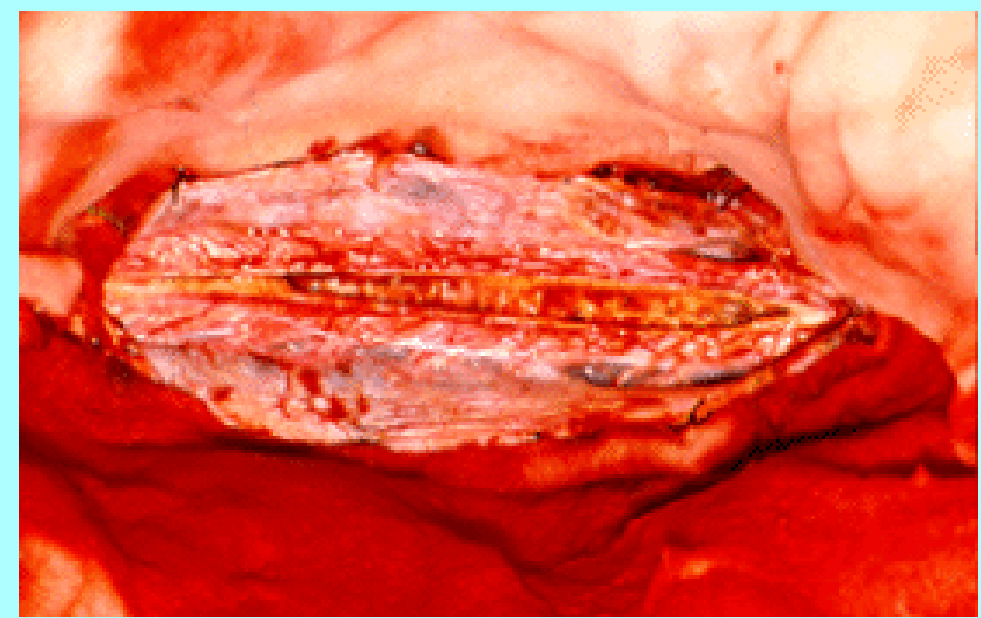

Fig. 24. Case 7. Intraoperative photograph obtained obtained after removal of the ependymomas. The photographic field is $20 \mathrm{~cm}$ in diameter.

Postoperative Course. He made excellent recovery from his spinal cord deficits and elected not to have reoperation of his partially removed right acoustic neuroma, nor any attempt to remove the left acoustic neuroma. Apparently he was correct in his decision, because there has been no significant progression in the past 10 years.

\section{CASE 8}

This 16-year-old Caucasian girl had complained of recurring stiff neck for approximately 8 years. Her orthopedist obtained cervical spine x-ray films 6 months prior to her admission to $\mathrm{MSH}$, and these were said to have been read as normal. Chiropractic manipulation begun 3 months prior to her admission made the pain worse. She was seen by an ophthalmologist who recognized papilledema, and an MR image revealed a huge ventral cervical spine tumor, extending from C-7 all the way up to the pontomedullary junction. She was put on full-dose decadron by her neurosurgeon and referred immediately to MSH.

Presentation and Neuroimaging Finding. On admission there was high-grade papilledema with hemorrhages but 20/20 vision. Her left pupil was sluggish and larger than the right, but very little else was detected on neurological examination. She fell to the right on tandem walking, but motor power, reflexes, and sensory examination were normal. Findings indicated that her cranial nerves were normal, including hearing bilaterally. There was one small café-au-lait spot and several small subcutaneous tumors, originally thought to be neurofibromas but later proved to be schwannomas. Studies including MR imaging of the head and entire spine showed a ribbonlike cervical cord stretched over a huge extramedullary ventral cervical tumor that went up the clivus to end in a mass in the right CPA (Fig. 25). There was also a 3-cm left acoustic neuroma and a number of very small neuromas scattered through the thoracic and lumbar spine. Surgical resection of the cervical cord tumor including the clival and right angle mass was planned. 


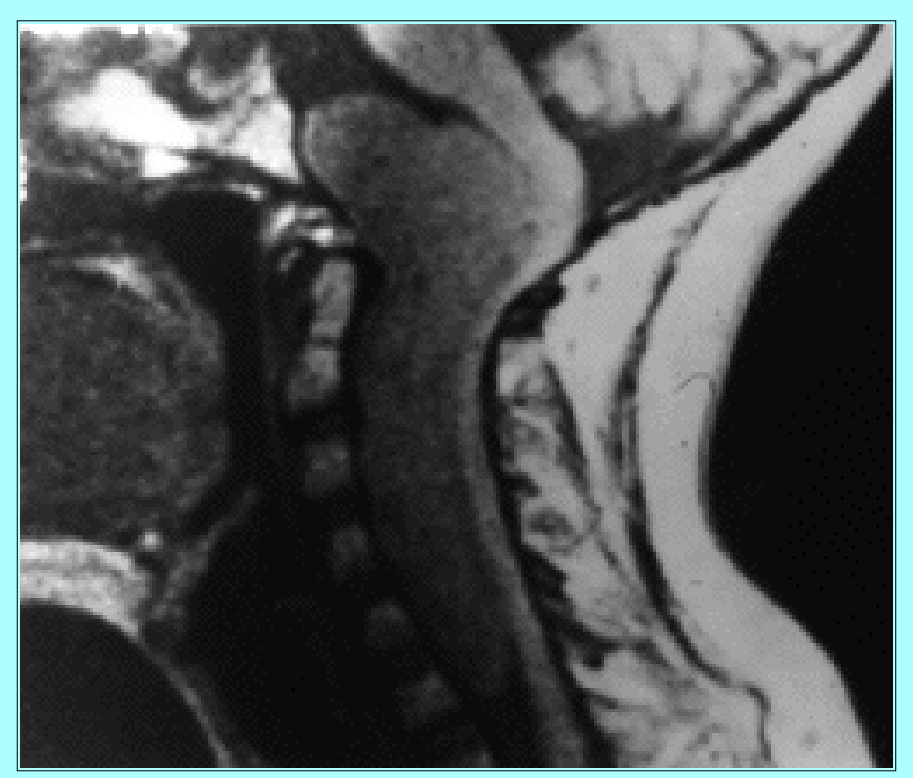

Fig. 25. Case 8. Contrast-enmhanced MR image showing a huge ventral schwannoma enlarging the cervical spinal canal. The tumor extends from the middle of the clivus down to C-7, causing remarkable distortion of the cervicomedullary junction.

Operation. This operative procedure, a right suboccipital craniotomy and total cervical laminotomy, was carried out in the semisitting position with all the standard arrangements as noted in the other cases reported here, except that the head was held in midposition, avoiding any flexion or extension.

The incision began just anterior to the right ear at the tragus and curved upward over the ear and then across to the midline and down the midline to the T-1 level of the spine. The incision was carried down through the galea. The galea edges were secured by hemostats and the scalp flap reflected in the subgaleal layer. The muscles were divided parallel to the scalp flap and reflected laterally in the suboccipital region, in the spine the midline incision was carried downward to expose the spinous processes and laminae through $\mathrm{C}-7$ by subperiosteal dissection. The exposure was maintained with modified Weitlaner retractors. The tremendously enlarged laminar arches were visualized, and exposure was carried out over the articular facets bilaterally. At the $\mathrm{C}-1$ through $\mathrm{C}-2$ levels on the right side, the tumor was at once seen extending out laterally through the space between the C-1 and C-2 lamina.

A suboccipital craniectomy was performed on the right side, including the entire mastoid process, using the high-speed 5-mm cutting burr; it was extended medially and inferiorly to include the foramen magnum. The craniectomy was brought across the entire sigmoid sinus and up over the lateral half of the right lateral sinus. The extension was sufficient to permit a presigmoid exposure if this were necessary. Despite the fact that the urea had been given, the exposed dura was incredibly tight and bulging. Intravenously administered furosemide was now used in attempt to allow further reduction in intracranial pressure while the laminotomy was carried out. Using the high-speed 2-mm rotary cutting burr, a lateral incision was made through the bone of the laminar arches of C3-6 on the right side, cutting through the medial part of the articular facets. A similar incision was brought down on the left side though here, because the enlargement of the canal was far greater on the right, the cut ran along the medial facet margin rather than through it. It was not possible to include C-2 or C-1 in this dissection because the head had been kept in midposition for fear of compression in flexion, and flexion would have been necessary to expose these levels with this resection. Now, by sharp dissection of the ligamentous attachments, the spinous processes and laminar arches of C3-6 were lifted out as a single structure and wrapped in saline sponges for future replacement. 
The exposed dura was nonpulsatile and tight, markedly expanded, and thinned. Using en face rongeuring, the laminectomy of C-1 and C-2 was now carried out, joining the open foramen magnum of the suboccipital craniectomy. This bony resection, however, did not apparently alter the drainage enough to permit any reduction of pressure. Resection of the C-7 spinous process and lamina arch was now carried out because it was obvious through the thin dura that the tumor extended past C- 6 into the C-7 level.

Up to this point, the operation had been carried out with 4 diameter loupes and the fiberoptic headlamps. These were now removed, and the remainder of the procedure was carried out through the Zeiss operating microscope at magnifications of 10 to 25 diameters. Because the dura was still so tremendously tight, a small incision was made just medial to the lower portion of the sigmoid sinus with a small bladed flexible retractor; the cerebellum was supported at this point so that it would not herniate and an opening was made in the arachnoid of the lateral portion of the cistern adjacent to the jugular foramen region. A free flow of CSF was achieved, and when this had continued sufficiently to reduce the pressure on the dura, it was possible to continue the dural opening. This was made as a question mark-shaped incision beginning inferior to the lateral sinus, curving around to the right to include the small dural incision already made, back to the midline at the foramen magnum, and then vertically down through the spinal canal to the T-1 level. The dura, arachnoid, and pia were largely attached together because of the tremendous enlargement resulting from this massive tumor. It was necessary to divide the adhesions between the dura and the arachnoid by microdissection. The dural margins were now sutured over the cut laminar edges to the adjacent muscles throughout the length of the spinal canal. Two lateral dural incisions were made from the suboccipital dural incision to the edge of the sigmoid sinus superiorly and inferiorly, and the lateral tab of dura was sewn with traction sutures out over the cut surface of the mastoid, drawing the sigmoid sinus partially out of the wound.

Dissection of the arachnoid was then carried over the entire exposed area, with arachnoidal trabeculae fused to the pia, and the vessels again requiring microdissection. The appearance in the spinal canal was rather remarkable: the spinal cord had been displaced backward and to the left as a thin ribbon with such great displacement that this ventral tumor had both the dorsal and ventral roots of the right side stretched over it. The dentate ligaments were so stretched out that they formed a membrane over $5 \mathrm{~mm}$ in width and paper-thin between the cord and lateral margins of the dentate where they were thicker; in the more medial portion of the stretched dentate they were quite transparent. The ventral nerve roots were thinned out between the paper-thin dentate and the tumor while the dorsal nerve roots lay tightly pressed against this area posteriorly. All of the dentates were cut from the highest dentate from C-1 down through C-7. This permitted entry into the tumor between the nerve roots at each level.

The first approach was carried out at the foramen magnum where the right cerebellar tonsil was elevated and distorted by a posterior-extending tumor of about $4 \mathrm{~cm}$ in diameter that appeared to arise from a portion of the spinal accessory nerve of the right side. This appeared to have a membranous connection to the rest of the mass but may well have been a separate tumor. The foramen magnum-area arachnoid was dissected, the right cerebellar tonsil lifted, and coring of this portion of the tumor was begun using the Malis cutting bipolar loops, working from the lateral portion of the tumor in order to devascularize, insofar as possible, the medial part of the tumor. This permitted progressive displacement of this portion of the tumor from behind the fourth ventricle and up into the medial part of the lateral recess where dissection from the posterior inferior cerebellar posterior branch was done; this segment of the tumor was separately delivered. After it was resected, it indeed appeared that this had been a tumor of the spinal accessory that had fused with the massive tumor, which appeared to have probably arisen from the right C-2 nerve root. 
Resection was now begun of the major spinal portion of the tumor. The tumor could be approached between each adjacent pair of right-sided nerve roots. Working at each level, again using the cutting bipolar loops, the tumor was cored laterally with the vascularity of the tumor from the dura controlled progressively as the coring was carried out. Originally the tumor had been quite vascular as well as dense and unsuckable. It was quite brightly yellow with many blood vessels within it and with a fibrous structure most suggestive of the plexiform neuroma but nevertheless more yellowish than one would expect for a neurofibroma, although much more fibrous than the usual schwannoma. As the lateral coring was carried out at every level, the tumor's consistency began to change with devascularization over the hours of dissection. It became still more yellow, now relatively avascular, and easier to core. The anterior-posterior diameter of the spinal canal at this point was more than $4 \mathrm{~cm}$, and it was possible to reach the ventral arachnoid, to progressively displace the tumor from medial to lateral, and then to continue the resection at each level in the same manner. At the level of the C-2 nerve root the mass extended out of the canal as already noted and this dumbbell-shaped extension was divided with the cutting bipolar loops, permitting the resection to be carried out further upward. The intradural extension of the tumor was followed up along the vertebral artery up into the posterior fossa, and the tumor mass was dissected away from the vertebral artery branches to the anterior spinal artery, from the origin of the PICA, the 12th cranial nerve, and the medullary branches of the 11th and the 10th cranial nerves. There was a reasonably good arachnoid layer superiorly protecting the AICA and the 7th and 8th cranial nerve complex.

It could now be demonstrated that, although the small right acoustic neuroma was fused to the lateral extension of the main tumor mass, this nodule arose from the superior vestibular medially and had simply displaced the auditory and facial nerves. There was minimal bulging into the IAC, which did not require drilling. With the resection of this portion of the tumor the entire spinal and ventral and right lateral portions of the tumor had been resected, except for the extension in the right C-2 nerve sheath. Additionally there had been tiny neuromas attached to many of the neural fascicles from the 11th cranial nerve down through the spinal canal. A number of these were also resected where they could be separated from their origin in cervical nerve roots and where individual neural fascicles, which became part of these tumors, could be resected with the tumor, while preserving the majority of fascicles of each root. In the posterior fossa there was known to be, on the opposite side a, radiologically well-defined left acoustic neuroma not visualized in this operative procedure.

Attention was now turned to the $\mathrm{C}-2$ right nerve root. The nerve root sheath extraspinally was split, and the tumor was resected from this area using the bipolar cutting loops to free this portion of the tumor from the vertebral artery. This had been a plexiform-like extension partly on the base of the skull. The sheath area was now filled with a block of gelfoam and covered with Surgicel. Dural closure was carried out for the entire spinal and suboccipital area using interrupted and continuous No. 4-0 Nurolon sutures. The dural surface was covered with a sheet of gelfoam and a Hemovac drain brought down to the gelfoam, as was an epidural camino pressure monitoring catheter. Drill holes were made in the lateral margins of the resected laminar arches and the adjacent facets of C3-6 and then the posterior laminar arch group was sewn into position with No. 2-0 Nurolon sutures. Muscles repair was done in layers with No. 2-0 Nurolon sutures attaching in part the lateral spinous muscles through the interspinous ligaments of the replaced lamina arches. Superiorly the galea was closed with inverted No. 3-0 Vicryl sutures while the deep spinal fascia was closed with No. 2-0 Nurolon and then the subcuticular layer in the spine closed with inverted Vicryl sutures. The skin was closed with staples throughout. The patient tolerated this 11-hour microsurgical procedure well. Blood loss was estimated at $500 \mathrm{ml}$. and was not replaced. 
She was brought to the ICU, was awake, extubated, and moving all extremities. All of the resected tumors were confirmed by neuropathological examination to be schwannomas. Recovery from this right suboccipital craniectomy and the cervical laminotomy had been excellent, with essentially no remaining deficits (Fig. 26).

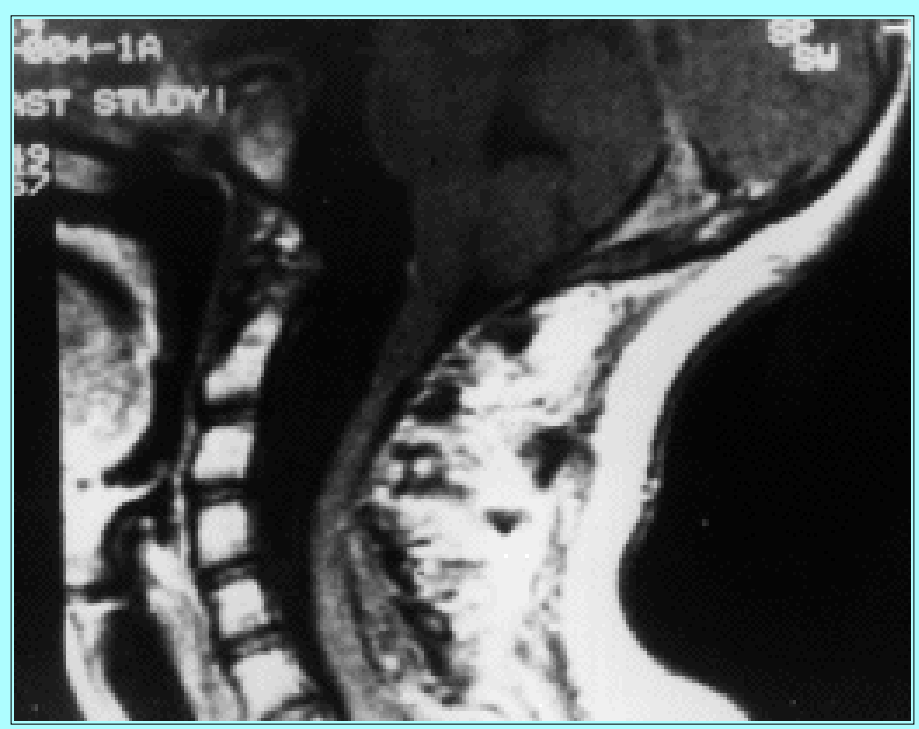

Fig. 26. Case 8. Postoperative MR image revealing that the tumor has been removed. Note the considerable reduction in the distortion of the spinal cord.

Second Operation. A few months later the patient experienced decreased hearing in the left ear. Repeated MR imaging showed the left acoustic neuroma to have increased to $3.5 \mathrm{~cm}$, and its removal was planned. This next operation, a left suboccipital craniectomy, was carried out in the semisitting position with all the arrangements noted in the other cases reported here. A vertical skin incision $8 \mathrm{~cm}$ long was made $1 \mathrm{~cm}$ medial to the left mastoid. The incision was carried down through the galea, deep fascia, and muscles to the suboccipital bone, and the mastoid was visualized by subperiosteal dissection. A 4-cm suboccipital craniectomy including the entire mastoid process was made with the 5-mm high-speed cutting burr. The sigmoid sinus was completely exposed. The mastoid cells were sealed with bone wax. The emissary vein was sealed with the bipolar coagulator and covered with gelfoam. A semicircular dural opening with its base medially was made, and lateral relaxing incisions were made, one up to the transverse and one down to the sigmoid sinus. Traction sutures were used to sew the lateral sinus out of the wound over the cut surface of the mastoid. The loupes and headlamps were removed and the remainder of the operative procedure was carried out under the Zeiss operating microscope at magnifications of 10,16 , and 25 diameters.

The large acoustic neuroma could be seen completely covering the ninth and 10th cranial nerves with exposure of part of the 11th cranial nerve through the arachnoid, so that the arachnoid was opened just beneath the line of the 11th cranial nerve, releasing the CSF. The Malis self-retaining brain retractor was placed to support the cerebellum in the plane of the petrous pyramid. The rest of the arachnoid dissection was carried out along the margin of the tumor as it bulged up to the tentorium and down toward the foremen magnum. Two abnormally large arcuate arteries came across above the tumor to reach the dura posterior to the IAM; they ran just beneath the unusually placed petrosal vein, which entered the petrosal sinus posterior to the meatus, preventing the cerebellum from separating from the tumor. It was necessary therefore to coagulate and divide the junction of the vein of the lateral recess and the petrosal vein medial to their entrance to the superior petrosal sinus. This permitted dissection of the arachnoid 
along the tumor as the cerebellum dropped away from it. Carrying the arachnoid dissection down from the tentorium to the lower pole of the tumor demonstrated that the tumor headed medially and inferiorly into the upper edge of the foramen magnum, obscuring the ninth and 10th cranial nerves completely.

The posterior surface of the tumor was highly vascular, with multiple arterial and venous channels networking on the capsule. These were stroked with the bipolar coagulator, and then the capsule was opened close to the petrous bone using the bipolar cutting current. The tumor's content was yellow-pink with a remarkable degree of vascularity most unusual for a neuroma, with good-sized vessels both arterial and venous within the tumor. Coring of the tumor required constant use of bipolar coagulation to seal and reseal these vessels as each segment of tumor was removed with the bipolar cutting loop forceps. The coring was carried out mainly inferiorly and laterally at first so that the tumor could be drawn upward a bit and so that the ninth and 10th cranial nerves could be visualized. As the arachnoid was dissected from these nerves, it could be seen that the fibers of the ninth and 10th cranial nerves were very markedly involved by very separate tumors. On the ninth cranial nerve one upper fibril had a 3- X 5 -mm separate nodule, whereas each of the other fibrils had fusiform tumors throughout much of their length. There would be short segments of almost normal-appearing nerves and then again fusiform enlargement. The spinal accessory nerve was entirely replaced by a fusiform bundle of about three times the normal width, coming up through the foramen magnum. Only the tiny tumor nodule on the ninth cranial nerve upper fascicle could be resected because the fusiform enlargements were unresectable without totally destroying the nerves.

Attention was again turned back to the acoustic neuroma, and as the coring continued upward a plane was found in which a second tumor, obviously arising from a different nerve or a different fascicle, had its capsule in dense contact with the inferior tumor. Nevertheless, there were neural fibers and vessels running between the two capsules. The superior tumor was also extremely vascular but was much harder, dense, and firm suggesting a neurofibroma much more than a schwannoma, although of course it was a schwannoma. Because this part of the tumor was cored, it was again exceedingly vascular. Finally, with continued removal, the apex of the tumor up past the fourth cranial nerve and tentorial margin, deeply displacing the fifth cranial nerve, was encountered and separated. The tumor extended through the tentorial notch compressing these structures and displacing the SCA. These were separated from the tumor and protected with pledgets as attention was again given to the lower pole of the tumor.

Coring of the lower pole now was carried out, freeing the tumor from the rest of the ninth cranial nerve with its incidental small tumors, following it medially over the auditory nerve to which it was densely adherent, and then following it along the vertebral artery and the PICA which were pushed downward and medially toward the foramen magnum by the tumor and then up along the BA. Here the brainstem had been displaced so far across the midline that the sixth cranial nerve had been drawn across the BA and had curved backward over the BA where it split into two fascicles: one superior to the origin of the AICA and the other inferior to it. The two fascicles of the sixth cranial nerve then came back to join as a single nerve adherent to the ventral tumor capsule up to the point where the sixth cranial nerve entered Durello's canal to go into the cavernous sinus. The sixth cranial nerve, BA, AICA, PICA, and vertebral arteries were all separated from the tumor without injury to any of these structures.

Now the facial nerve could be followed upward along the brainstem and this somewhat tan trunk curved forward below the fifth cranial nerve where it curved laterally and then began to thicken, obviously becoming part of a separate tumor. It was left in situ for the time being and the remainder of the medial part of the acoustic neuroma was resected to reach the margin of the IAM. Six months previously, 
imaging had demonstrated that this tumor in the left CPA had measured $2.5 \mathrm{~cm}$ in diameter. Four months later the tumor had measured $35 \mathrm{~cm}$, and it was this rapid enlargement that had precipitated surgery at this time. However, now just 6 weeks later, the actual measurement of the tumor in the operating room was just over $5 \mathrm{~cm}$, indicating a very rapid growth spurt. Additionally, 6 months previously, the small tumors of the ninth, 10th, and 11th cranial nerves had not shown up on gadolinium enhanced MR imaging; they were now clearly large enough to have been very obvious had a study been done again at this time confirming the rapid growth spurt.

The dura of the petrous pyramid posterior to the IAM was now coagulated with the bipolar and cut away to expose the petrous bone. Using the high-speed Aesculap drill, the posterior wall of the IAM was carved away to the limits of the tumor expansion, approximately $12 \mathrm{~mm}$ laterally and $9 \mathrm{~mm}$ vertically. The canal was filled with tumor over $1 \mathrm{~cm}$ in depth in the anterior-posterior direction. The tumor was cored using the 3-mm bayonet cup forceps, controlling bleeding with tiny pledgets or with bipolar current at 25 . As the tumor was progressively removed, it could be seen that both vestibular nerves right to the vestibular canals were part of the tumor so that tumor had arisen from both the superior and inferior vestibular nerves. The auditory nerve could be freed from the tumor, although it had clearly been so stretched and so involved that function of the remaining fibers, despite being preserved, was virtually impossible. The auditory artery, however, was freed from tumor and kept in good pulsating function. Several tiny branches from the auditory artery to tumor itself were sealed with the bipolar coagulator and divided. The facial nerve presented a major problem. There was clearly a separate tumor, fusiform in type, filling the facial nerve and expanding medially as it exited the canal to eventually wind up in continuity with normal-appearing nerve tissue where it had been displaced close to the fifth cranial nerve at the brainstem. Accordingly there was a segment of facial nerve approximately 3 to $4 \mathrm{~cm}$ in length that had the appearance of a fusiform neuroma extending all the way into the IAC and returning to relatively normal facial nerve appearance only as it entered the facial canal. Prolonged dissection on this segment of tumor, taking away bit by bit the portions of tumor that appeared to have grown out of the fusiform bundle, gradually reduced its diameter to approximately 7 or $8 \mathrm{~mm}$; however, this remaining segment of nerve was clearly totally filled with fusiform tumor. Accordingly, this portion of the tumor of the facial nerve was left in situ as the ninth, 10th, and 11th cranial nerves had been. This completed the tumor removal. Separate specimens had been sent from the various areas for pathological evaluation. Hemostasis had been complete, although the procedure of obtaining hemostasis throughout the removal had been difficult and prolonged. The brainstem, despite the long dissection, was indeed completely normal in appearance although deeply cupped.

The drilled out area in the IAC was filled with a fat pad from the subcutaneous layer, welded into place to the dura with the bipolar coagulator set at 12 , and the irrigation turned off. The self-retaining brain retractor was removed and the cerebellum allowed to return to its normal position. The cerebellum appeared to be untraumatized. Dural closure was carried out using No. 4-0 Nurolon. The dural surface was covered with a sheet of gelfoam. A Hemovac drain was brought in through a separate stab wound to the epidural surface. Using the nitrogen-powered 1-mm burr, a groove was cut in the diploe of the craniectomy all around, except over the mastoid, and a sheet of titanium mesh was bowed into the groove to restore the previous bone contour. The mesh was then spread with acrylic cranioplastic material that was allowed to polymerize under constant irrigation. The usual layer closure of the wound was carried out using No. 2-0 interrupted Nurolon in multiple layers for the muscles and deep fascia and No. 3-0 interrupted Vicryl for the galea and subcuticular layer. The skin was closed with skin staples. Blood loss was estimated at $300 \mathrm{ml}$. and was not replaced. The patient tolerated this 6-hour microsurgical procedure 
well and was sent to the ICU in satisfactory condition, reacting and breathing well after reversal and extubation.

Again the patient made an excellent recovery, although with loss of hearing in the left ear. The left facial area was initially very weak, requiring installation of a gold weight in the upper lid to permit eye closure, but this improved rapidly. The right ear had adequate hearing for her to resume her normal activities.

For 2 years after the removal of the left acoustic neuroma the patient got along reasonably well, with a minimum of difficulty. Then lumbar spine symptoms led to laminectomy and removal of two cauda equina schwannomas (Fig. 27). Magnetic resonance imaging then demonstrated a right posterior falx meningioma $1.5 \mathrm{~cm} \mathrm{X} 2 \mathrm{~cm}$ and a right petroclivotentorial meningioma $1.5 \mathrm{~cm}$ in diameter (Fig. 28 left). One year later the falx meningioma had grown to $2 \mathrm{X} 3 \mathrm{~cm}$, and the petroclivotentorial meningioma had grown to $2.5 \times 3 \mathrm{~cm}$, diminishing the hearing in her right ear to the point at which a hearing aid provided little benefit. Additionally, there were innumerable smaller tumors on the dural surface, forming a generalized meningiomatosis. Despite all of this, she was working and driving her car.

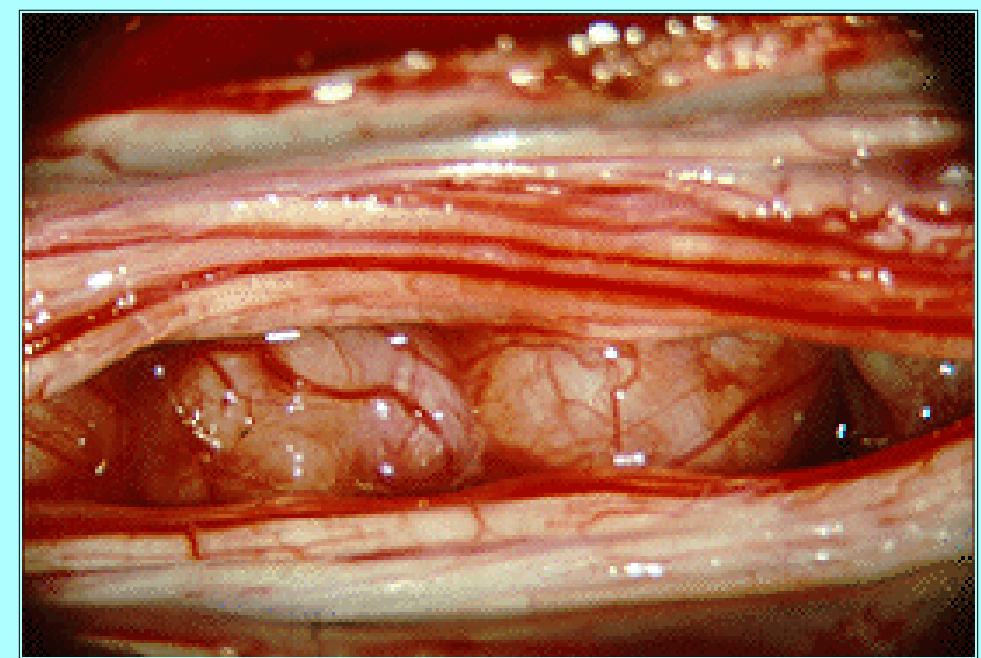

Fig. 27. Case 8. Intraoperative photograph showing two round schwannomas in the cauda equina. The photographic field is $36 \mathrm{~mm}$ in diameter.

Six months later, 4 years after her laminotomy and first craniectomy, her condition rapidly deteriorated; she needed help in walking, was totally deaf, and had difficulty swallowing. Magnetic resonance imaging showed tremendous enlargement of the right petroclivotentorial meningioma with additional increase in the other tumors (Fig. 28 center and right). As a last resort it was decided to attempt resection of the petroclivotentorial tumor.
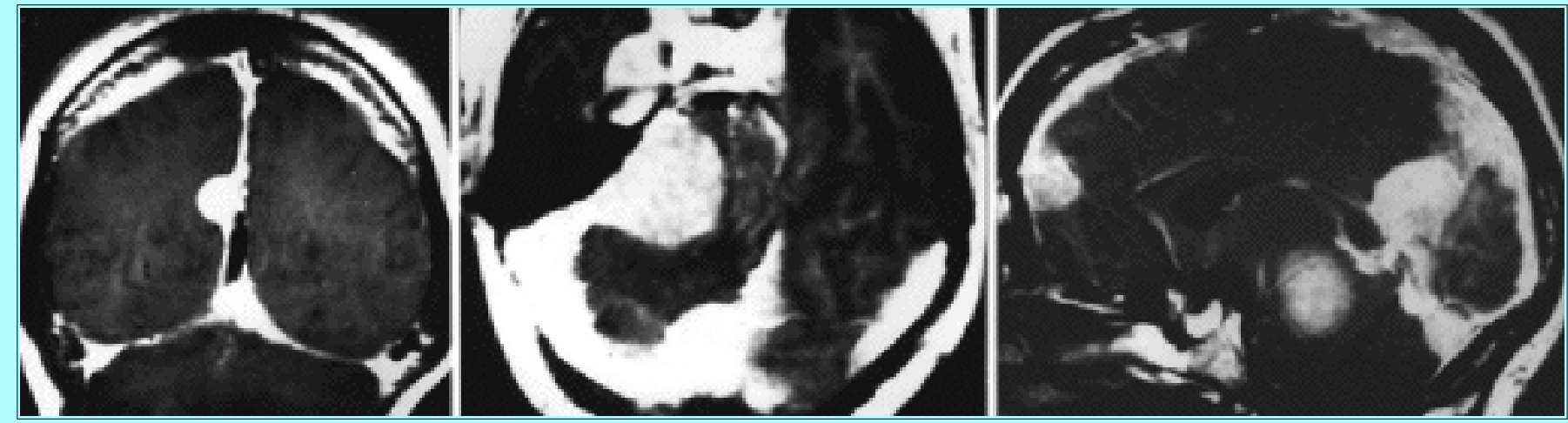

Fig. 28. Case 8. Left: An MR image demonstrating calvarial, falx, and tentorial dural 
thickening, which was considered to be due to meningomatosis, and a small right posterior falx meningioma. Center: An MR image demonstrating a right petroclivotentorial meningioma extending onto the lateral sinus and the torcula and up the falx, anteriorly on the right cavernous sinus, and onto the left posterior fossa dura. Right: A sagittal MR image confirming the extensive meningiomatosis, which is also on the sagittal sinus.

Third Operation. This operation, a petrosal temporal craniotomy and secondary suboccipital craniectomy, was carried out in the semisitting position with all the arrangements noted in the other cases reported here. There was a well-healed scar of the incision, beginning just anterior to the right tragus, curving upward and turning around over the pinna, and curving backward to reach to the midline and down the cervical region. The anterior part of this well-healed old scar was reincised, the incision carried above and then down to the area of the foramen magnum, and the scalp flap reflected downward and forward. The scarred area of the old craniectomy was now dissected free to visualize the previously exposed lateral sinus and sigmoid sinus, as well as the lower area of the temporal dura and the closed previously opened dura of the posterior fossa. The bony margin of the petrous apex laterally was resected with a high-speed cutting burr down to within $5 \mathrm{~mm}$ of the external auditory meatus. The dura was opened along the temporal floor, and although scarred, appeared reasonably normal, as was the underlying brain surface. Labbe's vein was followed to the transverse sinus and the superior surface of the tentorium exposed. The dura of the posterior fossa, which was so thickly scarred, was now opened with a linear incision parallel to the sigmoid sinus, with extensions curving parallel to the posterior fossa floor as close to it as feasible and another curving parallel to the inferior surface of the lateral sinus. The inner surface of the dura here, however, was invaded by an underlying meningioma. Resecting a small amount of the meningioma at the junction of the lateral sinus and the upper end of the sigmoid sinus between the entrance of the superior petrosal sinus and the entrance of Labbe's vein permitted a temporary clamp to be placed across the venous sinus junction. Observation then demonstrated that there was no increase in bulging of either temporal lobe or cerebellar area. Accordingly, a suture of $O$ silk was placed through the tentorium around the sinus and tied to occlude the sinus. Another suture was passed just inferior to this one, and the sinus was divided between the suture ligatures. The tentorium was divided progressively upward along the line of the petrous apex 1 or $2 \mathrm{~mm}$ medial to the superior petrosal sinus. The tumor was immediately seen to be involving the superior petrosal sinus.

A plane of cleavage was now achieved posteriorly between the cerebellum and the posterior surface of the tumor. This was about $2.5 \mathrm{~cm}$ from the invaded sigmoid. The plane of cleavage permitted the placement of cottonoid pledgets for a depth of about $2 \mathrm{~cm}$, which gave some protection to permit the coring of the meningioma. This was begun along its dural attachment laterally and along the tentorium using the cutting bipolar forceps loops to resect cores of the tumor, devascularizing the tumor almost bloodlessly. This was carried inward along the petrosal sinus with the resection of the invaded superior petrosal sinus, exposing the upper edge of the petrous bone at its junction where the tentorium had been divided as well. Meanwhile it was clear that as one extended the dissection downward, the entire area of the jugular foramen and of the IAM was completely covered by tumor; a radical resection along this area would involve the resection of all of the arterial and neural structures related to these foramina. Based on the the imaging studies it was known that a meningiomatous carpet lined all of the posterior fossa and the upper calvarium as well. This lining varied from 1 to $3 \mathrm{~mm}$ in thickness, and from it the outgrowth of multiple larger meningiomas had occurred. Because this was a known restriction to removal, the dissection of the tumor in the angle was carried approximately 3 to $4 \mathrm{~mm}$ in thickness from the underlying bone, neural structures, and foramina. 
As the lateral coring continued along the tentorium, to the notch, and downward along the floor carpet of meningioma, it was possible to progressively remove the main mass of the tumor from medial to lateral and to keep repeating the coring process. Eventually the lateral surface of the pons was reached and protected with cottonoid patties while the cerebellum was supported by the Malis self-retaining flexible retractor without pressure on the neural structures. By this point in the dissection much of the tumor, which had had its blood supply from the lateral surface, had been relatively devascularized, and it was now possible to debulk the interior of the tumor more rapidly. Despite the extensive invasion of the tumor, it was possible to achieve reasonable arachnoid planes along the pons, thereby protecting pontine vasculature and flattened-out neural structures that ran downward to go beneath the layer of carpeted-floor meningioma. Finally, posteriorly a knob of tumor entered the foramen magnum, and this knob had only arachnoid attachments without dural invasion. It extended down from the other portion of the tumor and was not a separate nodule in any way. After coring, it was possible to lift the capsular layer out of the foramen magnum, dissect it from the arachnoid overlaying the vertebral artery, and remove this segment completely. Here, for the first time, there occurred a free flow of CSF welling up from below when the final segment of the tumor was removed. This completed the subtotal removal of this massive meningioma, leaving in place the carpeting layer against the floor of the posterior fossa, along the posterior portion of the petrous pyramid, and its supratentorial extension toward the cavernous sinus. The carpetlike layer had been made totally hemostatic by stroking with the bipolar coagulator at low powers, but additionally a strip of Surgicel was placed over the exposed meningiomatous carpet as security against future oozing.

Dural closure was now carried out using No. 4-0 Nurolon. A Hemovac drain was brought in through a separate stab wound to the epidural surface and the dural surface was covered with a sheet of gelfoam. The usual layer closure of the wound was carried out using No. 2-0 interrupted Nurolon in multiple layers for the muscles and deep fascia and No. 3-0 interrupted Vicryl for the galea and subcuticular layer. The skin was closed with skin staples. Blood loss was estimated at $300 \mathrm{ml}$. and was not replaced. The patient tolerated this 9-hour microsurgical procedure well and was sent to the ICU in stable condition, reacting well after reversal and extubation (Fig. 29).

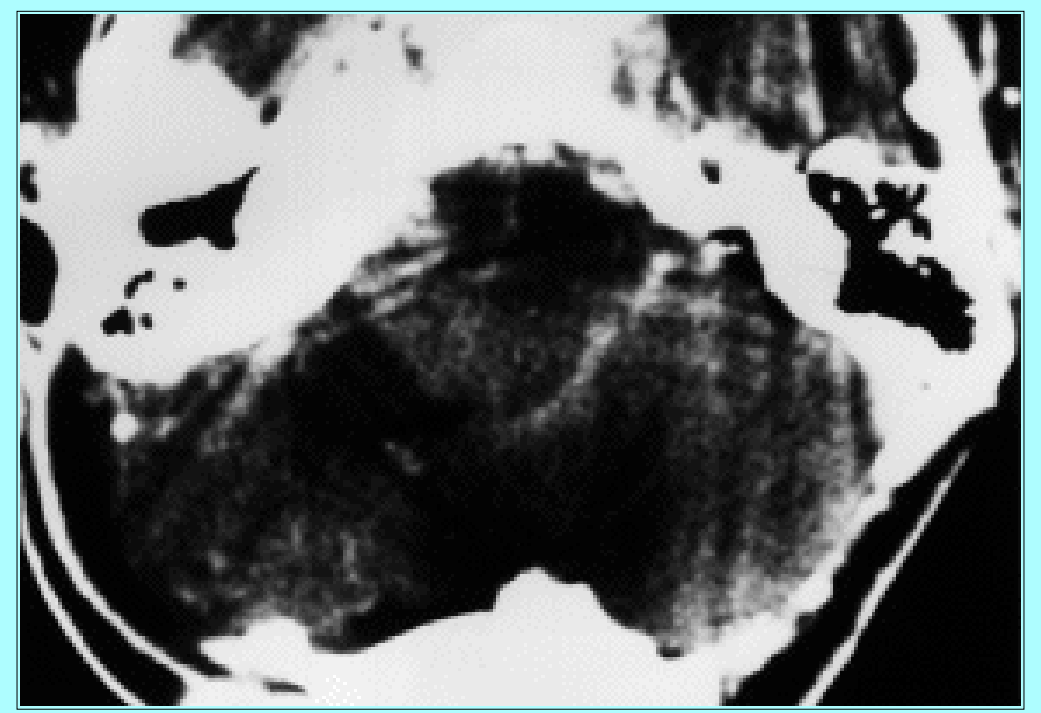

Fig. 29. Case 8. Postoperative MR image demonstrating the extent of meningioma resection.

Postoperative Couse. She made a reasonable recovery, considering the massive involvement and the expected phenomenally rapid growth rate that had been observed, and was able to be taken home for convalescence. After only a few months, her condition deteriorated again, with further meningioma 
growth causing occlusion of the sagittal sinus down through the torcula Herophili, and she died just over 4 years from her original surgery.

\section{CASE 9}

History. This 46-year-old Caucasian man became totally deaf progressively while a student years before. This was called sensory neural deafness and a CT scan in 1975 is said to have shown no cause. He remains totally deaf but is an excellent lip reader, he is married, and has two healthy children. He lost his sense of smell completely some years ago. In the past year or two he has had increasing right arm pain, first more severe at night and finally occurring during the daytime as well, and it is at times excruciating.

A CT scan of the head and cervical spine demonstrated bilateral small acoustic neuromas, a trigeminal neuroma, and multiple other neuromas as well as a foramen magnum meningioma, a large subfrontal meningioma ( $3 \times 6 \mathrm{~cm})$, and small optic sheath meningiomas. Additionally there was thickened enhancement of all of the dura of the cranium and cervical spine. Considered to be most important at the moment was cord compression at $\mathrm{C} 1-2$, with the cord reduced to a narrow ribbon between the two intradural tumors, considered to be most likely neuromas.

Presentation. He was referred and admitted to MSH for foramen magnum and upper cervical laminectomy. On admission, despite the lesions demonstrated on CT scanning, he had been working full time. On examination he had normal mental status, normal visual acuity and fields, and normal gait. He was totally deaf and anosmic. There was weakness of the right trapezius, sternomastoid, and deltoid, and minimal weakness of the right arm as compared to the left.

Operation. The operative preparation was comparable to that already noted for the other procedures in this series except that neck flexion could not be considered. The patient was brought into the semisitting position with the back elevated only 30š and the head supported without tension and without pressure in exact midposition. Somatosensory evoked potential recording was attempted first in the supine position, but there was no response above C-6.

A midline linear incision was made from the skull base to the spinous process of C-6 and brought down to expose the tips of the spinous processes. The deep fascia was incised, and the spinous process and laminae of C1-5 were visualized. The exposure was maintained with a modified Weitlaner retractor. A wide bilateral laminectomy of the articular facets and pedicles was carried out of C1-5. The cut bony edges were waxed, and gelfoam strips were placed in the lateral gutters. A burr opening was made in the suboccipital area low on the right side. After dural separation, a 5-cm wide and 3-cm high suboccipital craniectomy was carried out including the foramen magnum rim. The loupes and headlamps were removed, and the remainder of the operative procedure was carried out under the Zeiss operating microscope at viewed field diameters from 1.6 to $3.6 \mathrm{~cm}$.

A transverse dural incision was made along the upper edge of the suboccipital craniectomy going from right to left. The situation was immediately unique. The arachnoid was completely replaced by a several-millimeter thick layer of tissue which looked like a carpet of fused multiple meningiomas but was lacking in psammoma bodies. The incision was carried across to the left side and then downward as a reversed question mark to then come back to the midline at the foramen magnum and then downward through the spinal canal. The same thick layer apparently replaced the arachnoid and completely covered the inner surface of the dura throughout the entire laminae that had been resected, as well as the entire suboccipital dura. The vascularity in the membrane was sealed with the bipolar coagulator as the cut was 
carried down. The dural margins were sutured over the cut bony edges with No. 2-0 Nurolon, and this required sharp dissection of the arachnoid trabeculae, which were still intact and appeared normal between the surface of the cord and the surface of the abnormal arachnoidal-replacing membrane.

In the center of the posterior fossa, separating the tonsils and pushing the upper cord and lower medulla forward, was a globular $2.5 \mathrm{~cm}$-typical meningioma that was attached posteriorly to the dura and thus began to displace laterally and posteriorly as the dural incision was carried out. The tumor's vascular supply and adherence was to the heavy layer of abnormality, and although it was a smoothly and capsulated round tumor, there was no actual separation between it and the point of arachnoidal membrane to which it had been attached (Fig. 30).

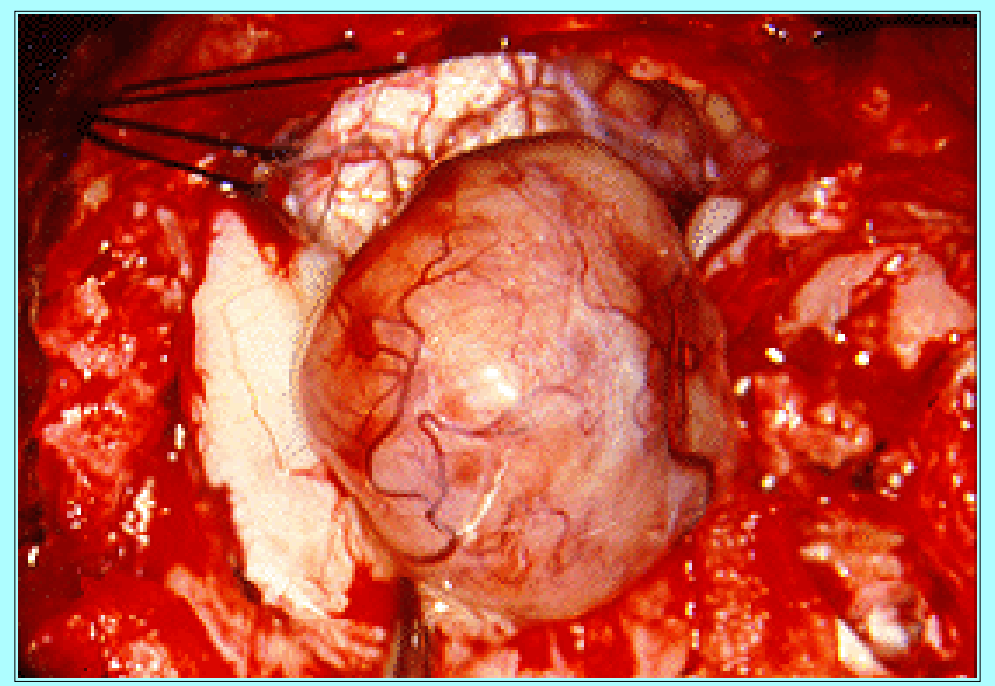

Fig. 30. Case 9. Intraoperative photograph of a $\mathrm{C}-1$ meningioma. The photographic field is $36 \mathrm{~mm}$ in diameter.

Inspection of the cord permitted the evaluation of the rest of this most remarkable situation. The cord at C1-2 and at C2-3 was a thin ribbon compressed mainly from the anterior but also from the sides to form a sort of thinned triangle approximately 4 or $5 \mathrm{~mm}$ in width and less than $3 \mathrm{~mm}$ in anteroposterior thickness by large masses bilaterally present anterolaterally at these two levels (Fig. 31 left). The largest mass was at C1-2 on the right and this was the first to be approached. The dentate ligaments above and below were cut, the dissection carried along the arachnoidal thick tumor membrane until the root foramen was reached, and here the root appeared to be part of the tumor. Dissection from the root entry zone medially was followed to where it entered the tumor. Attempts at dissection along the root within the tumor demonstrated that the root was totally adherent as part of the sheath and was indeed part of the tumor. Accordingly the C-2 root was divided fascicle by fascicle after the fascicles were sealed with the bipolar coagulator. The dissection was continued laterally, and it became clear that the entire ventral root was also a part of the tumor which was drawn backward at the root sleeve and divided where it became nonneoplastic. It was now possible to begin to bring the tumor laterally out from beneath the cord, separating its attachments which were virtually entirely to the lateral ventral arachnoid and not to the pia of the cord. The tumor was resected. This tumor was about $2 \mathrm{X} 1.5 \mathrm{~cm}$ in diameter and was white to tan in color with a poorly defined capsule; it appeared more likely to be a schwannoma than a meningioma, although it was not that different in its color or texture from the lining of the canal, which had replaced the arachnoid. Attention was turned to the left C1-2 area where a slightly smaller tumor approximately $1.5 \mathrm{~cm}$ in diameter presented in exactly the same way and was resected in quite the same manner, again removing the ventral and dorsal $\mathrm{C}-2$ roots. 


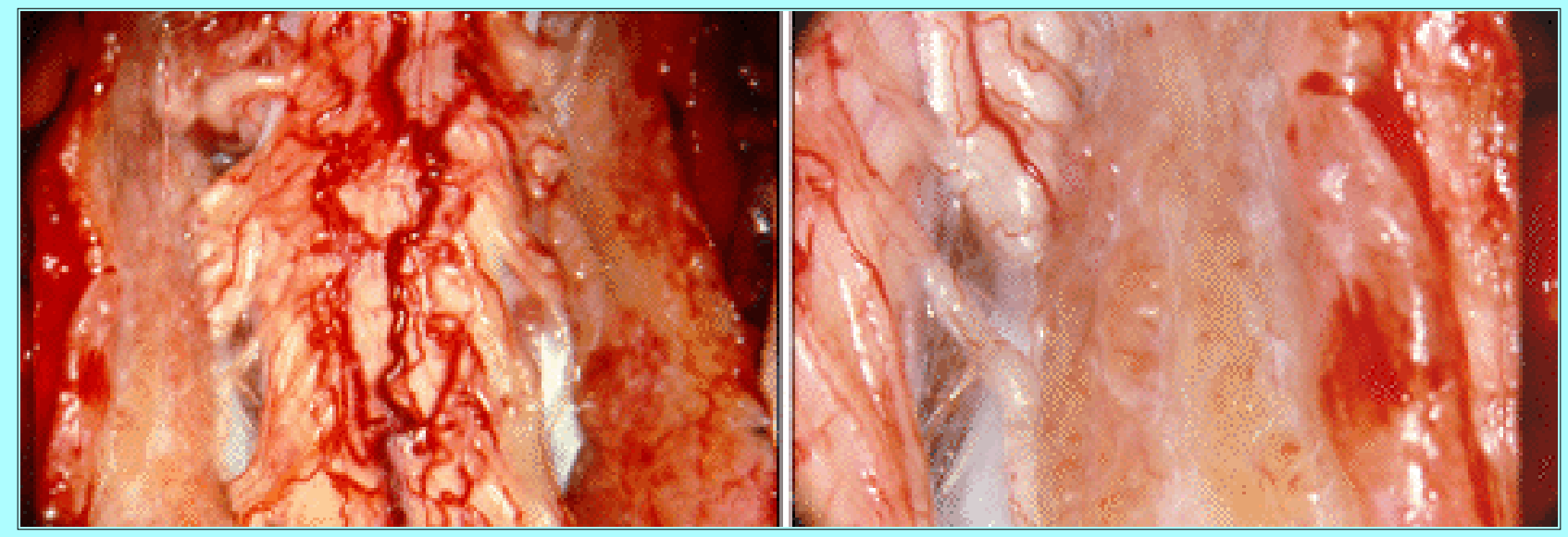

Fig. 31. Case 9. Intraoperative photographs showing that the arachnoid at C-4 has been replaced by a thin carpet of meningiomatosis. The dura has been sutured to muscle laterally and all dentates have been cut (left). The photographic field is $36 \mathrm{~mm}$ in diameter. Right: The same view at higher magnification showing the right edge of the spinal cord with the nerve roots running downward and the abnormal membrane on the right (right). The photographic field is $18 \mathrm{~mm}$ in diameter.

With the removal of these tumors at $\mathrm{C} 1-2$, the ribbon of cord was perfectly free and floated forward and backward over the remaining ventral large bulge at C2-3. These dentates were divided, and the tumor on the right side at C2-3 was dissected. Again the dorsal roots were followed into the tumor until they clearly became inseparable, despite the use of the highest magnification and the finest dissection. The tumor was firm, hard, and totally unsuckable. Whereas its appearance was quite like that of those in the C1-2 area, its hardness was more characteristic of meningioma than neuroma. The dorsal root of C-3 was divided, fascicle by fascicle, and then, working laterally again, the foramen was cleared only by removing the total root. Now it was possible to roll out the tumor from beneath the cord so that the ventral root fascicles could be divided medially. This right side C2-3 tumor was removed. On the left side at C2-3, again almost the same situation was present. The tumor was removed in the same manner. It was slightly smaller, but the difference was negligible.

There was still a bulge of the cord right at the center of the C2-3 level. Rotating the cord by traction on the dentate ligaments demonstrated that this was from an additional mass that lay completely ventrally, slightly to the right at the C-2 level where it had been actually between the curves of the ventral surfaces of the right-sided tumors already removed. It arose from the thick arachnoid tissue tumor membrane and was not attached to a nerve root, yet it looked similar to the other four tumors already removed from the spinal canal. Dissection around the arachnoid attachment into this tumor, mainly with the bipolar coagulator, permitted the bloodless removal of this ventral mass, and it too was sent as specimen. This completely decompressed the cord.

Continuing at the C-3 and C-4 levels the tumors were significantly smaller bilaterally, and it was possible to remove them without sacrificing the nerve roots. Fortunately, this permitted the removal because resection of the C-4 ventral roots would have paralyzed the diaphragm. Here the tumors again were enlargements and excrescences coming from this arachnoid-thickened layer. The C4-5 level had no major tumor mass but only the thick layer which, at this point, had thickened to between 3 and $4 \mathrm{~mm}$ but was without a particular enlarged segmental rounding. On the other hand, at C5-6 on the right side there was a significant further enlargement, again coming from this arachnoid layer, and this was resected without any root resection. 
All of the remaining dentate ligaments of the exposed cord were brought out into view and cut bilaterally. Rather remarkably, all of these dentate ligaments were completely free of the infiltrated material that covered the arachnoid, and there was no infiltration of any of the pial surfaces (Fig. 31 right). A segment of the arachnoid was dissected free from the dura, and this piece of arachnoid, approximately $2 \mathrm{~cm}$ long and $1 \mathrm{~cm}$ wide and $3 \mathrm{~mm}$ thick, was sent as a separate specimen for neuropathological evaluation. It was obvious on looking through the foramen magnum that the entire arachnoid up into the posterior fossa had this same character, and this explained the unusual enhancement of the arachnoidal dura over the entire surface of the brain on the CT scan. This was, of course, unresectable because it covered the entire head and certainly the entire cervical spine circumferentially.

Again looking up through the foramen magnum opening, the nerve roots here appeared to be thickened in places as though they contained neuromas but were without discrete neuromas that could be resected; however, two small filaments of the 11th cranial nerves, one on each side, had in continuity within them oval football-shaped tumors perhaps 4 or $5 \mathrm{~mm}$ in length and $3 \mathrm{~mm}$ in diameter. These were resected, taking with them only the fascicle that involved them and leaving the 11th cranial nerve root essentially intact bilaterally. These two filaments were also separately sent for neuropathological evaluation and were confirmed as schwannomas, whereas all of the other lesions were meningiomas with an unusual inflammatory component.

Because the circumferential, thick, abnormal arachnoid layer could not be totally resected, it was decided to leave the dura sutured to the muscles and to replace the posterior dura with gelfoam as an artificial dura. This was done leaving the sutures in permanent place to hold the dura widely open and by then placing a double sheet of gelfoam, one just within the sutured layer and the other just outside of it, curving in the opposite direction from the muscles. A Hemovac drain was brought in through a separate stab wound to the gelfoam layer, and then the deep tissues were closed with No. 2-0 interrupted Nurolon sutures in anatomical layers and an additional layer of No. 2-0 Nurolon was used for the deep fascia. The galea above and the subcuticular layer below were closed with inverted interrupted No. 3-0 Vicryl sutures, and the skin was closed with staples. The patient tolerated this procedure well and, after reversal, was extubated with good respirations, both diaphragmatic and thoracic, and with movement in all four extremities returning as the reversal progressed. The patient was then moved to the ICU in excellent, stable condition.

Postoperative Course. He made an excellent recovery and returned to work essentially free of the symptoms that led to the need for surgery, but 1 year later he experienced recurrence of shoulder pain and some leg weakness. Neuroimaging demonstrated increasing encroachment on the lower cervical cord by increased thickening of the meningiomatosis with more nodular enlargement. This required a repeated cervical laminectomy, with more tumor removal and wider decompression. No end to this patient's problem is yet in sight.

\section{CASE 10}

Presentation and Neuroimaging Findings:This 17-year-old Caucasian boy first presented with a left facial palsy and bilateral hearing loss. On admission to MSH he was otherwise apparently normal, with no stigmata of NF. Angiography revealed large bilateral acoustic neuromas, and chest and spine images demonstrated an 8-cm mediastinal mass, apparently dumbbelling from the left T2-3 foramen. It was decided to carry out sequential removals, beginning with the mediastinal mass, then to do interval 
surgery on the CPA tumors.

Operation. With the patient in the 45š prone oblique position with the left side up, using a longer than usual muscle exposure, the spinous processes and laminae from T1-5 were visualized, and a full laminectomy was performed on T1-4. A large epidural neuroma centered at T-3 was found, tremendously enlarging the foramen on the left between T-2 and T-3, and separating the rib heads. The T-2 and T-3 left pedicles were resected with the articular facets. The second and third rib heads were resected with $4 \mathrm{~cm}$ of the ribs. This exposed the mediastinal tumor adequately. The removal was begun by progressive coring. When coring had provided sufficient room, the epidural part of the tumor was dissected free, with the T-2 nerve root sheath entering the mediastinal portion (Fig. 32 left). The nerve sheath was ligated with No. 2-0 Nurolon close to the dura and divided distally. The plane between the tumor capsule and the pleura was dissected using microsurgical technique, and the entire capsule was excised after dividing the distal normal area of the nerve root. The pleura was intact, and no chest tube was needed.
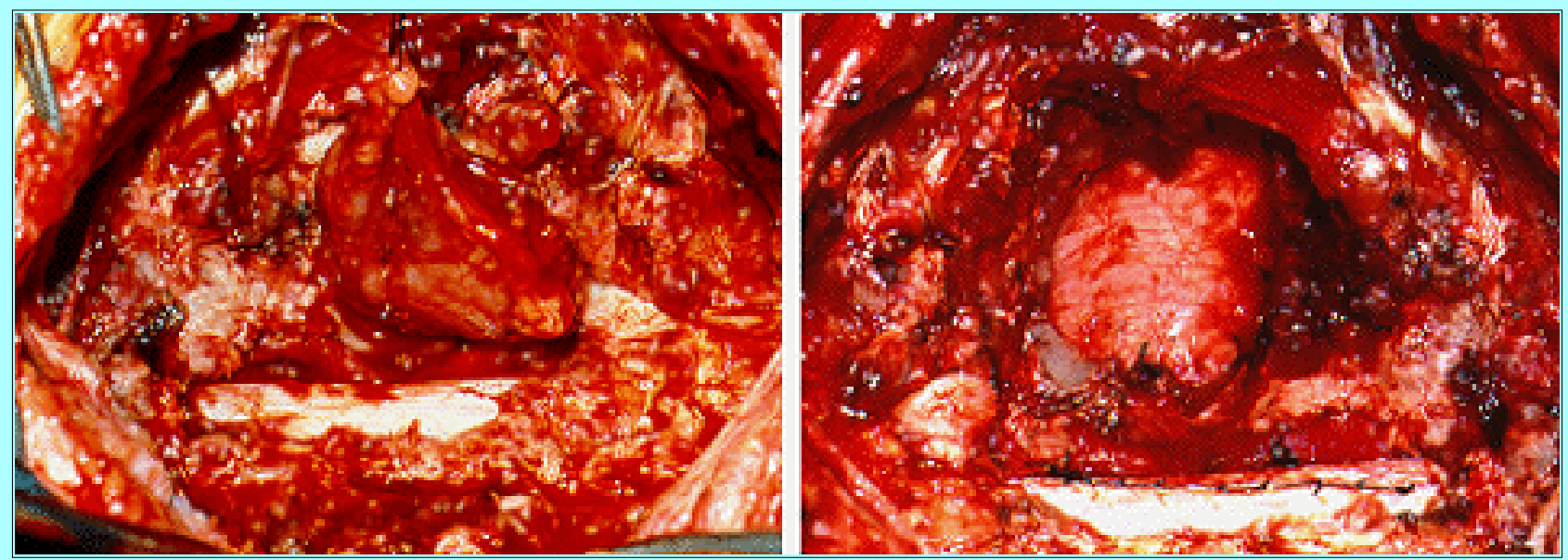

Fig. 32. Case 10. Intraoperative photographs showing a partially dissected mediastinal tumor which has been reflected medially to laterally with the portion that was epidural now at the upper edge of the photograph. The white dura is seen near the lower edge (left). The tumor has been removed and the unopened pleura can be seen, as can the line of the continuous suture on the dura (right). The photographic field is $24 \mathrm{~cm}$ in diameter.

The dura was then opened with a midline incision, and a 1-cm intradural extension of the neuroma was resected after dividing the T-2 nerve root medially. The sheath was ligated again after completely emptying. An addition small neuroma was noted on the T-3 nerve root, unconnected with the other tumor, and this was also removed. The dura was closed with a continuous suture of No. 4-0 Nurolon (Fig. 32 right), and the dura and the pleura were covered with a sheet of gelfoam. The usual layer closure procedure of muscle, fascia, and cutaneous layer was carried out. No stabilization procedure was considered necessary.

Second Operation. The patient had no symptoms preoperatively from this tumor, and none appeared postoperatively. Two months later he was readmitted for left suboccipital craniectomy, the side of the facial paralysis. The usual preparation in the semisitting position and the opening were done as in the other cases in this series. After the arachnoid opening and dissection and the placement of the self-retaining flexible retractor, the ninth, 10th and 11th cranial nerves were seen at the jugular foramen. All were studded with multiple neuromas, with every nerve completely engulfed at some point (Fig. 33 left). 


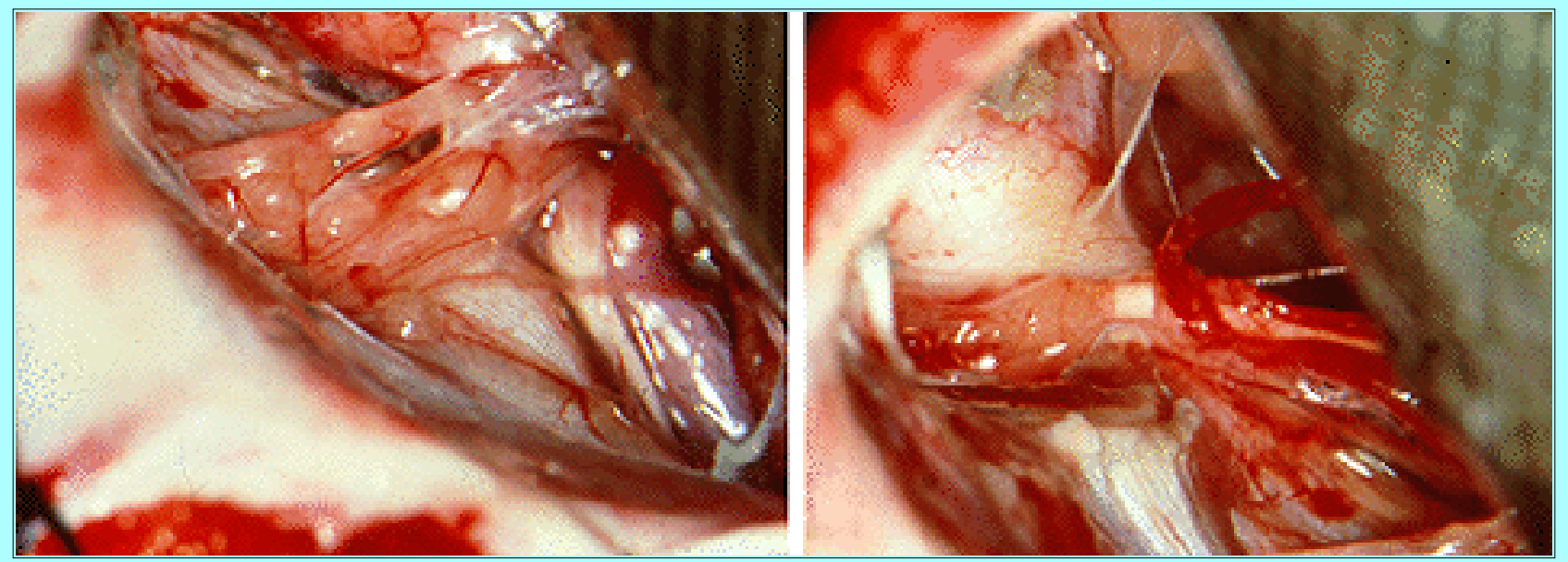

Fig. 33. Case 10. Intraoperative photographs obtained during a left suboccipital craniectomy in which the ninth, 10th, and 11th cranial nerves were found to be studded with multiple small schwannomas at the jugular foramen (left). The photographic field is is $24 \mathrm{~mm}$ in diameter. The acoustic neuroma is being cored, and at a depth of $1 \mathrm{~cm}$, another capsule was encountered, surrounded by the first neuroma (right). The photographic field is $36 \mathrm{~mm}$ in diameter.

Coring of the acoustic neuroma was begun using the cutting bipolar and bayonet cup forceps. Within the capsule the tumor was yellow, fatty, and relatively avascular. Just $1 \mathrm{~cm}$ beneath the surface, a second capsule was encountered, with surface vessels and neural fibers between the outer tumor and this inner capsule (Fig. 33 right), suggesting a multicentric origin. Tracing all of the neural fibers in this unusual situation could not be accomplished, but this 4-cm total mass was removed out to the IAM with preservation of the facial nerve fibers, which had run in a curved course on the anteroinferior surface of the outer capsule of the tumor. The IAC was drilled open out to the crista and found to be filled with tumor. As this part of the tumor was resected piecemeal, the facial nerve was found to be totally engulfed in a very small separate fusiform neuroma (Fig. 34 left). This neuroma was removed with the facial nerve because the patient had preoperative total facial paralysis. I was unable to find a suitable proximal facial nerve segment for primary anastomosis or graft. The canal was filled with fat, the dura closed, and the usual wound closure procedure was done.
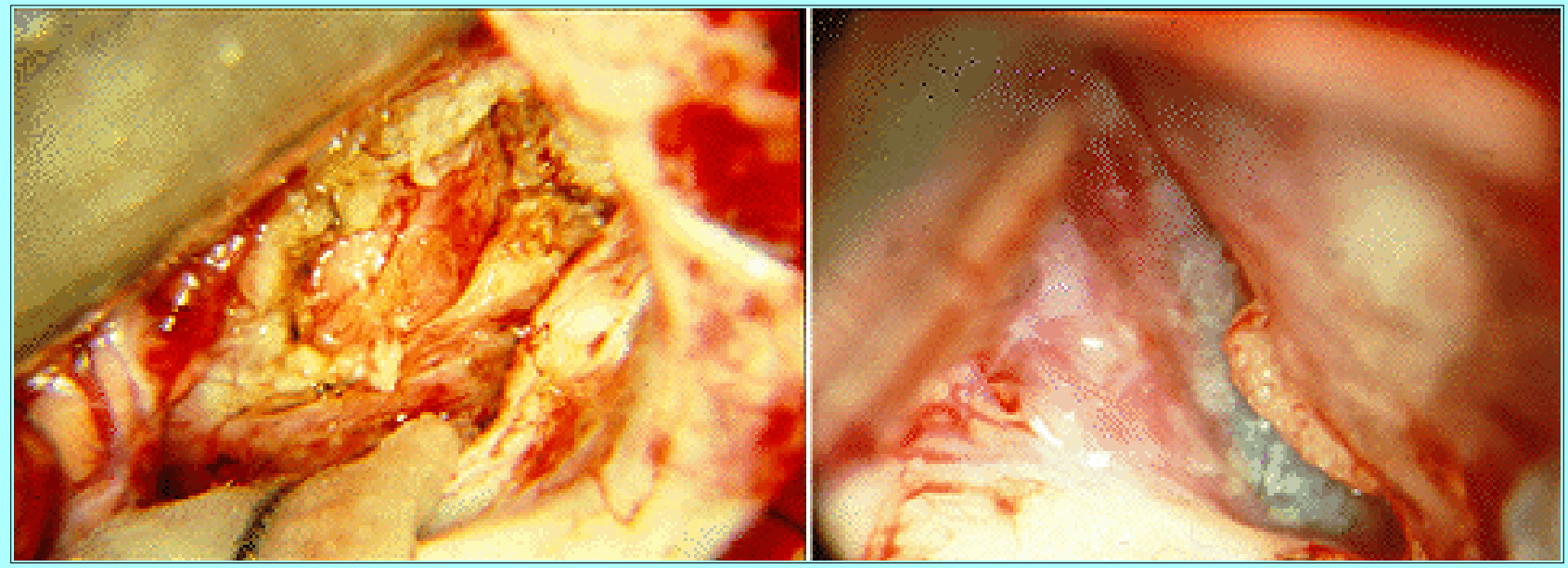

Fig. 34. Case 10. Left: Intraoperative photograph obtained during a left suboccipital craniectomy in which the acoustic neuroma was removed and the internal auditory canal opened. Note the separate fusiform neuroma engulfing the facial nerve. Right: Intraoperative photograph obtained during a right suboccipital craniectomy in which a tiny tentorial edge 
meningioma was removed. The fifth cranial nerve is at the lower edge of the picture. The photographic field is $24 \mathrm{~mm}$ in diameter.

Third Operation. Several months after an excellent recovery, aware that the much decreased hearing in his right ear would probably be completely lost, the patient underwent a right suboccipital craniotomy. This was a straight forward procedure, quite like an acoustic neuroma removal in a non-NF2 patient, with one exception: there was a tiny meningioma on the tentorial edge at the notch (Fig. 34 right). This was removed with the bipolar forceps and the dural margin was thoroughly coagulated. The facial and auditory nerves were preserved, but although facial function was saved, he was now totally deaf bilaterally.

Postoperative Course. For treatment of the facial paralysis, I am unwilling to do 12-7 anastomosis in NF2 patients because bilateral 12th cranial nerve loss has a fatal outcome, and although I have not seen 12 th cranial nerve neuromas in my series, I have seen 12 th cranial nerve loss due to meningiomas of the skull base. Because there were neuromas of the 11th cranial nerve, an 11-7 anastomosis did not seem reasonable. A plastic surgical fascial support procedure was done with satisfactory appearance at rest.

It is now 20 years since his tumors were removed, and most amazingly, none of his remaining tumors have enlarged and no new ones have been detected. He has completed college, reads lips and signs well, and works full time.

\section{DISCUSSION}

A major problem exists in the decision regarding when and whether to operate on the side of the remaining hearing ear when hearing has been lost on the other side, particularly when the remaining tumor is small and not immediately incapacitating. In my series, patients who presented with bilateral acoustic neuromas in their 30s are more likely to have a long and stable life expectancy than patients who present in childhood. There is no question that the better the hearing in an ear with an acoustic neuroma, the better is the probability that it can be saved. It is generally true that the smaller the tumor, the less likely it is to interfere with hearing, and the easier and less traumatic it is to remove. This would make a very logical case for early operation. However, where this has certainly been true in my own series of solitary acoustic neuromas, it has not been true in my patients with NF2. Preservation of hearing with removal of the neuroma in this group of patients has been a dismally unhappy experience for me.

In my 600 surgically treated patients with non-NF2 acoustic neuromas, there are 128 who had speech discrimination of $80 \%$ or better, and only $30 \mathrm{db}$ of decreased hearing amplitude. These are patients who can use the telephone in the affected ear. Of this group I have been able to preserve the hearing at a useful level in 69 cases, approximately 50\%. On the other hand, in the NF2 patients who so desperately need the ability to hear in one ear, my results have been much poorer. Of the 57 acoustic neuromas I have resected in the NF2 patients, there were 28 acoustic neuromas in patients whose hearing was adequate preoperatively, whereas only four retained adequate hearing postoperatively, approximately $15 \%$. Some hearing, enough to aid in lip reading, was preserved in four other patients. The depression experienced by staff, the surgeon, and of course the total feeling of devastation in the patient who goes into the operating room early with a small tumor hoping to have hearing but comes out totally deaf is a terrible experience. A previous partial removal appears to reduce the odds still further, both in solitary acoustic neuromas and in the bilateral acoustic neuromas in NF2.

I maintain that the use of focused beam or any other form of radiation, whether gamma knife, linac, or 
proton beam, is indicated only in an exceptional few of these NF2 patients. I use focused beam radiation therapy in patients who have utterly hopeless situations in which all that can be hoped for is short-term palliation. I ordinarily reserve radiotherapy for patients who are quite elderly and infirm or for whom life expectancy is so short that palliation is acceptable or for those whose tumors are absolutely inoperable. For younger people, such as most of the patients in the NF2 group, I believe that curative treatment of any tumor is to be the goal. Of my 41 patients with bilateral acoustic neuromas, many have been followed for more than 20 years. In addition to their acoustic neuromas I have removed a total of 208 tumors from of other areas of brain and spinal cord in these patients, and 28 of the 41 patients continued to lead productive lives.

I have seen a significant number of patients, mostly cases of pituitary tumor, with radiation-induced meningiomas, which occurred in the irradiated areas of dura more than 20 years after the radiation therapy. A great proportion of NF2 patients have, or develop, meningiomas and certainly do not need, if they have a prolonged survival, the occurrence of additional radiation-stimulated tumors. With the high spontaneous incidence of meningiomas, no study is likely to prove or disprove the risk. These induced meningiomas might arise wherever dura has received fairly high doses of radiation. This is certainly true if one is treating the IAC with radiotherapy.

Many of the NF2 patients have multiple small tumors on the ninth, 10th, and 11th cranial nerves and tumors embedded in the posterior root of the fifth cranial nerve. These tumors often cannot be seen on neuroimaging and would not be targeted in the radiation therapy. They are readily visualized at surgery, and many of them can be removed in the course of the acoustic neuroma resection without traumatizing the main trunks while they are at this tiny size.

In my 41 patients there are 14 acoustic neuromas on which I have not operated. These 14 patients have adequate hearing in their only hearing ear. I have continued to follow them in the hope that they will retain their hearing for a long time and that their tumors will not grow too rapidly before they have to be removed. It has been suggested that these patients learn lip reading or signing before surgery. In my experience this has been completely unsuccessful and not one patient has learned either until the loss occurred.

Over all, of the 82 acoustic neuromas (in 41 patients with NF2), prior surgery had been performed elsewhere in 16 tumors, of which 11 had been partly removed and only five had been totally removed. Between 1975 and 1994 I operated on 57 of these tumors, including reoperation on five of the previous partial removals. All 57 operations achieved total removal. Of the previous partially removed tumors, six have not yet been redone. In 14 acoustic tumors in 41 patients being followed, not yet operated in, the tumor involves the only remaining hearing ear.

In this same group of patients, in addition to the acoustic neuromas, I have removed 208 other significant tumors, not counting many tiny nodules. From the posterior fossa there were 22 meningiomas and 61 other neuromas. From the supratentorial compartment there were 11 meningiomas and one tuberous sclerosis. I have removed 99 extramedullary tumors from the spinal canals, of which 41 were meningiomas and 58 were schwannomas. There were 14 intramedullary spinal cord tumors removed of which nine were ependymomas, three were astrocytomas, and two were central schwannomas.

There were no deaths resulting from surgery in this series, but four late deaths occurred, three as a result of uncontrollable rapid growth of multiple intracranial meningiomas in quite young patients and one unexplained. 
Of the 41 patients, 28 are working or at school. One patient has postoperative paraplegia. There are six patients who were blind when first referred, one from bilateral retinoblastoma resected in childhood, one from suprasellar meningioma, and four as a result of papilledema (two from supratentorial meningioma, and two from their acoustic tumors). These last four became blind before their diagnosis was made and before undergoing neurosurgical evaluation.

The explosion of genomic research in the last 20 years has produced an almost logarithmic increase in publications with development of an overwhelming amount of data, too much for most clinicians to even contemplate reading. Chromosome 22 alone can readily become a full-time specialty study. I shall briefly summarize what I have considered the most important from my clinician's standpoint, with the reservation that the next few years will produce remarkable change. Neurofibromatosis type 2 is the result of an abnormal gene on chromosome 22 at Q12, with a resulting defect in the production of the tumor-suppressor protein schwannomin, also called Merlin. Growth rates in NF2 are extremely variable. Schwannomin is a 595 amino acid protein. With only single amino acid abnormality, the disease is mild, whereas frameshift deletions or insertion and nonsense mutations causing truncation of schwannomin cause more severe disease. Severe disease cases appearing in childhood with large numbers of crippling and fatal tumors are at one extreme, whereas mild cases with slow-growing and fewer tumors appear later in life, although there are exceptions. It appears that this difference is related to the differences in the NF2 gene.

If a father-to-be has NF2 and the mother does not, amniocentesis or chorionic biopsy to test for the abnormal NF2 gene might be reasonable if the parents were willing to abort if positive tumors were recognized. If abortion were an unacceptable alternative, then I do not believe conception is appropriate. At the time of this writing, although feasible, the tests are simply not readily available but certainly will be in the near future. If the woman has NF2, pregnancy is so likely to produce exacerbation of her tumor growth that I believe child bearing should be avoided. I have spent long hours counseling patients and families about this and have brought in trained advisors as well, with remarkably little effect on their behavior.

Nevertheless, this group of patients has simply been remarkable in their courage and optimism, their cheerfulness and readiness to support each other and to carry on in face of adversity. Several patients have made a policy of checking to determine when a patient recently diagnosed with NF2 was coming in and would come to visit and help with the adjustment. When they knew a patient had lost hearing one or two would arrive to demonstrate how well they could get along with their deafness, and they would send cards and letters. In a very special way they came to be for me and some of my staff like an extension of family.

The hope is that the fabulous development of genomic knowledge will culminate in the ability to replace Merlin or the gene that produces Merlin. Never having to subject these very special people to the repeated injury caused by their tumors and by surgical treatment would be a virtual miracle, but considering what has been discovered in the last decade alone, this result must soon be achieved.

\section{Suggested Bibliography}

1. Allen JC, Eldridge R, Young, D: Early-onset acoustic neuroma: genetic, clinical and nosologic aspects. Birth Defects 10:171-184, 1974 
2. Antinheimo J, Haapasalo H, Haltia M, et al: Proliferation potential and histological features in neurofibromatosis 2-associated and sporadic meningiomas. J Neurosurg 87:610-614, 1997

3. Baser ME, Mautner VF, Ragge NK, et al: Presymptomatic diagnosis of neurofibromatosis 2 using linked genetic markers, neuroimaging, and ocular examinations. Neurology 47:1269-1277, 1996

4. Brasfield RD, Das Gupta TK: Von Recklinghausen's disease: a clinicopathological study. Ann Surg 175:86-104, 1972

5. Davis GB, Berk RN: Intestinal neurofibromas in von Recklinghausen's disease. Am J Gastroenterol 60:410-414, 1973

6. Dutcher PO Jr, House WF, Hitselberger WE: Early detection of small bilateral acoustic tumors. Am J Otol 8:35-38, 1987

7. Evans DG, Mason S, Huson SM, et al: Spinal and cutaneous schwannomatosis is a variant form of type 2 neurofibromatosis: a clinical and molecular study. J Neurol Neurosurg Psychiatry 62:361-366, 1997

8. Gardner WJ, Frazier CH: Bilateral acoustic neurofibromas: a clinical study and field survey of a family of five generations with bilateral deafness in thirty-eight members. Arch Neurol Psychiatry 23:266-302, 1930

9. Gusella JF, Ramesh V, MacCollin M, et al: Neurofibromatosis type 2: loss of Merlin's protective spell. Curr Opin Genet Dev 6:87-92, 1996

10. Gutmann DH, Aylsworth A, Carey JC, et al: The diagnostic evaluation and multidisciplinary management of neurofibromatosis 1 and neurofibromatosis 2. JAMA 278:51-57, 1997

11. Halliday AL, Sobel RA, Martuza RL: Benign spinal nerve sheath tumors: their occurrence sporadically and in neurofibromatosis types 1 and 2. J Neurosurg 74:248-253, 1991

12. Harding AE: Clinical and molecular neurogenetics in neurosurgery. Adv Tech Stand Neurosurg 20:81-104, 1993

13. Harkin SC, Reed RJ: Tumors of the Peripheral Nervous System. Atlas of Tumor Pathology, Ser 2, Fasc 3. Washington, DC: Armed Forces Institute of Pathology, 1969

14. Hilal SK, Solomon GE, Gold AP, et al: Primary cerebral arterial occlusive disease in children. II. Neurocutaneous syndromes. Radiology 99:87-94, 1974

15. Hughes GB, Sismanis A, Glasscock ME, III, et al: Management of bilateral acoustic tumors. Laryngoscope 92:1351-1359, 1982

16. Huson SM, Thrush DC: Central neurofibromatosis. Q J Med 55:213-224, 1985

17. Kanter WR, Eldridge R, Fabricant R, et al: Central neurofibromatosis with bilateral acoustic neuroma: genetic, clinical and biochemical distinctions from peripheral neurofibromatosis. Neurology 30:851-859, 1980

18. Kluwe, L, Mautner VF: A missense mutation in the NF2 gene results in moderate and mild clinical 
phenotypes of neurofibromatosis type 2. Hum Genet 97:224-227, 1996

19. Korf BR: Neurocutaneous syndromes: neurofibromatosis 1 , neurofibromatosis 2 , and tuberous sclerosis. Curr Opin Neurol 10:131-136, 1997

20. Malis LI: Acoustic tumor, in Pillsbury HC III, Goldsmith MM III (eds): Operative Challenges in Otolaryngology: Head and Neck Surgery. Chicago: Yearbook Medical, 1990, pp 77-91

21. Malis LI: Electrosurgery. Technical note. J Neurosurg 85:970-975, 1996

22. Malis LI: The etiology of disruption of the facial neuromuscular motor unit--surgical causes: in the cranium, in Rubin LR (ed): Reanimation of the Paralyzed Face. St. Louis: Mosby, 1977, pp 57-70

23. Malis LI: The facial nerve in tumors of the angle, in Portmann M (ed): Proceedings of the Fifth International Symposium on the Facial Nerve. New York: Masson, 1985, pp 70-72

24. Malis LI: Instrumentation and techniques in microsurgery. Clin Neurosurg 26:626-636, 1979

25. Malis LI: Intra-operative monitoring is not essential. Clin Neurosurg 42:203-213, 1995

26. Malis LI: Microsurgical treatment of acoustic neuromas, In Handa H (ed): Microneurosurgery. International Symposium on Microneurosurgery. Baltimore: University Park Press, 1975, pp 105-120

27. Malis LI: Nerve grafting by microscope in the cranium, in Rubin LR (ed): Reanimation of the Paralyzed Face. St. Louis: Mosby, 1977, pp 211-216

28. Malis LI: Neurofibromatosis (von Recklinghausen's disease), in Cummings CW (ed):

Otolaryngology-Head and Neck Surgery. St. Louis: Mosby, Vol 4, 1986, pp 3449-3456

29. Malis LI: Neurosurgical photography through the microscope. Clin Neurosurg 28:233-245, 1981

30. Malis LI: New trends in microsurgery and applied technology, in Pluchino F, Broggi G (eds): Advanced Technology in Neurosurgery. Berlin: Springer-Verlag, 1988

31. Malis LI: Prevention of neurosurgical infection by intraoperative antibiotics. Neurosurgery 5:339-343, 1979

32. Malis LI: Titanium mesh and acrylic cranioplasty. Neurosurgery 25:351-355, 1989

33. Martuza RL: Genetic factors in brain tumors, in Wilkins RH, Rengachary SS (eds): Neurosurgery. New York: McGraw-Hill, 1985, Vol 1, pp 501-511

34. Martuza RL: Genetics in neuro-oncology. Clin Neurosurg 31:417-440, 1983

35. Martuza RL: Neurofibromatosis and other phakomatoses, in Wilkins RH, Rengachary SS (eds): Neurosurgery. New York: McGraw-Hill, 1985, Vol 1, pp 511-521

36. Martuza RL, Ojemann RG: Bilateral acoustic neuromas: clinical aspects, pathogenesis and treatment. Neurosurgery 10:1-12, 1982

37. Matthies C, Samii M: Management of 1000 vestibular schwannomas (acoustic neuromas): clinical presentation. Neurosurgery 40:1-10, 1997 
38. Moyes, PD: Familial bilateral acoustic neuroma affecting 14 members from four generations. Case report. J Neurosurg 29:78-82, 1968

39. Nager GT: Association of bilateral eighth nerve tumors with meningiomas in von Recklinghausen's disease. Laryngoscope 74:1220-1261, 1964.

40. Ojemann RG: Management of acoustic neuromas (vestibular schwannomas). Clin Neurosurg 40:498-535, 1993

41. Riccardi VM, Kleiner B: Neurofibromatosis: a neoplastic birth defect with two age peaks of severe problems. Birth Defects 13:131-138, 1977

42. Ruttledge MH, Andermann AA, Phelan CM, et al: Type of mutation in the neurofibromatosis type 2 gene (NF2) frequently determines severity of disease. Am J Hum Genet 59:331-342, 1996

43. Samii M, Matthies C, Tatagiba M: Management of vestibular schwannomas (acoustic neuromas): auditory and facial nerve function after resection of 120 vestibular schwannomas in patients with neurofibromatosis 2. Neurosurgery 40:696-705, 1997

44. Samii M, Turel KE, Penkert G: Management of seventh and eighth nerve involvement in cerebellopontine angle tumors. Clin Neurosurg 32:242-272, 1985

45. Seizinger BR, Martuza RL, Gusella JF: Loss of genes on chromosome 22 in tumorigenesis of human acoustic neuroma. Nature 322:644-647, 1986

46. Wilkins RH, Brody IA: Von Recklinghausen's neurofibromatosis. Arch Neurol 24:374-377, 1971

47. Yasargil MG: Microsurgery of CNS tumors. Microneurosurgery, Vol IVB. New York: Thieme, 1996, pp 100-124

48. Young DF, Eldridge R, Gardner WJ: Bilteral acoustic neuroma in a large kindred. JAMA 214:347-353, 1970

Manuscript received February 24, 1998.

Address reprint requests to: Leonard I. Malis, M.D., 219-44 Peck Avenue, Hollis Hills, New York City, New York 11427. 